\title{
Select Polyphenol-Rich Berry Consumption to Defer or Deter Diabetes and Diabetes-Related Complications
}

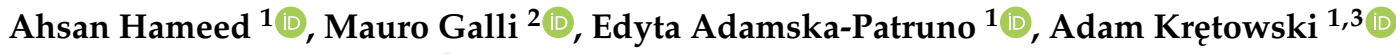 \\ and Michal Ciborowski ${ }^{1, *(1)}$ \\ 1 Clinical Research Center, Medical University of Bialystok, 15-089 Bialystok, Poland; \\ ahsan.hameed@umb.edu.pl (A.H.); edyta.adamska@umb.edu.pl (E.A.-P.); adamkretowski@wp.pl (A.K.) \\ 2 Department of Medical Biology, Medical University of Bialystok, 15-222 Bialystok, Poland; \\ mauro.galli@umb.edu.pl \\ 3 Department of Endocrinology, Diabetology, and Internal Medicine, Medical University of Bialystok, \\ 15-089 Bialystok, Poland \\ * Correspondence: michal.ciborowski@umb.edu.pl
}

Received: 11 June 2020; Accepted: 11 August 2020; Published: 21 August 2020

\begin{abstract}
Berries are considered "promising functional fruits" due to their distinct and ubiquitous therapeutic contents of anthocyanins, proanthocyanidins, phenolic acids, flavonoids, flavanols, alkaloids, polysaccharides, hydroxycinnamic, ellagic acid derivatives, and organic acids. These polyphenols are part of berries and the human diet, and evidence suggests that their intake is associated with a reduced risk or the reversal of metabolic pathophysiologies related to diabetes, obesity, oxidative stress, inflammation, and hypertension. This work reviewed and summarized both clinical and non-clinical findings that the consumption of berries, berry extracts, purified compounds, juices, jams, jellies, and other berry byproducts aided in the prevention and or otherwise management of type 2 diabetes mellitus (T2DM) and related complications. The integration of berries and berries-derived byproducts into high-carbohydrate (HCD) and high-fat (HFD) diets, also reversed/reduced the HCD/HFD-induced alterations in glucose metabolism-related pathways, and markers of oxidative stress, inflammation, and lipid oxidation in healthy/obese/diabetic subjects. The berry polyphenols also modulate the intestinal microflora ecology by opposing the diabetic and obesity rendered symbolic reduction of Bacteroidetes/Firmicutes ratio, intestinal mucosal barrier dysfunction-restoring bacteria, short-chain fatty acids, and organic acid producing microflora. All studies proposed a number of potential mechanisms of action of respective berry bioactive compounds, although further mechanistic and molecular studies are warranted. The metabolic profiling of each berry is also included to provide up-to-date information regarding the potential anti-oxidative/antidiabetic constituents of each berry.
\end{abstract}

Keywords: berries; metabolic syndrome; precision nutrition; hyperglycemia; hyperlipidemia; diabetes; omics; metabolomics; genomics

\section{Introduction}

Diabetes mellitus (DM) is a multifactorial disease with high mortality worldwide. Chronic DM is the eighth-leading cause of deaths globally, responsible for 1.5 million deaths each year [1]. According to the World Health Organization (WHO), in 2013, 381 million adults were diagnosed with DM, which increased to 422 million in 2016 and is expected to double by 2030. Type 1 (T1DM) represents $15 \%$ of cases, and the remaining cases are type 2 (T2DM) [1]. T2DM is primarily treated with pharmacotherapeutic drugs, evidence-based alternative approaches, and functional food-based approaches [2]. Pharmacotherapeutic approaches generally consist of monotherapy 
or binary/poly-therapy, depending on severity. Most physicians use the binary approach and prescribe insulin-secretogenic sulfonylurea drugs and the insulin sensitivity enhancer metformin. Additional drugs address diabetes-induced vascular complications, with the average number of prescribed daily drugs being as high as four [3]. Combined drug therapy is associated with long-term side-effects and other costs, resulting in non-adherence [4]. Moreover, evidence-based alternative approaches may have safety and toxicity issues due to which precision nutrition-based approaches have recently been proposed as alternatives to defer or deter T2DM and its complications.

The provision of individualized dietary and nutritional recommendations is referred to as precision nutrition. Polyphenol-rich fruits (including berries) are the primary components of precision nutrition, and consumption of these fruits, like berries, represent a potential "frontline strategy" for combating T2DM in obese or overweight patients. Substantial evidence suggests that T2DM onset can be prevented or managed by berries and/or berries-derived-tailored dietary intake, exercise, and the maintenance of healthy body weights (BWs) [5]. Therefore, targeted berries-nutrition is considered analogous to an individualized medicinal approach, providing effective and safe nutritional interventions for DM prevention and management. Furthermore, the American Diabetes Association and Dietary Guidelines for Americans also strongly recommend diets rich in anthocyanin and polyphenols to protect against and manage DM [6]. Increasing evidence shows that berry consumption also reduces DM risk, including a recent study showing that Finnish men who regularly consumed berries reduced their T2DM risks by up to 35\% [7]. Due to the significance of berry consumption and the lack of comprehensive studies examining berry consumption effects specifically on DM, this study aimed to collect and summarize all studies examining the relationship between berry consumption and DM.

$\mathrm{DM}$ is a metabolic syndrome with concordance changes in insulin sensitivity and/or availability. This insulin insensitivity and/or deficiency induces derangements in metabolic pathways related to glucose, lipids, and protein metabolism. Berry, or its byproduct, intake not only opposes these derangements by normalizing the metabolic homeostasis of glucose, lipids, and protein metabolism, but also improves insulin sensitivity and secretary indexes. Therefore, all available in vitro and in vivo studies involving whole berries or berry bioproduct consumption and citing the normalization of insulin signaling, secretion, and sensitivity, restoring the altered glucose, lipid, and protein metabolism, and reduction of oxidative stress and inflammatory cytokines were included. In order to determine the hypoglycemic and hypolipidemic potential of berries, studies that added berries to high-fat (HFD) and high-carbohydrate (HCD) diets, defined as diets with $>45 \%$ fat and $>60 \%$ carbohydrates, respectively, were also included. In addition to HFD and/or HCD, disruption of intestinal endothelium and homeostasis resulting in epithelial inflammation, increased permeability (i.e., dysbiosis), and alteration in gut microbial taxonomic composition and diversity (increase in Firmicutes:Bacteroidetes ratio, and reduction in intestinal mucosal barrier dysfunction (IMBD) restoring bacterial families, proteolytic and glycolytic microflora, short-chain fatty acids (SCFA), and organic acids (SCOA) producing microflora) are also considered risk factors to obesity and DM. IMBD associated bacterial families protect the epithelial layer of the intestine whereas SCFA and SCOA played important role in the synthesis and production of immunoglobulins and immune-supportive cytokines to protect against dysbiosis and metabolic disorders. In this context, the impact of berry or berry product intake on the attenuation of obesity-associated disorders and dysbiosis was also reviewed. Studies involving the metabolic fingerprinting of berries were also described to represent the possible number of compounds considered responsible for their antioxidative and antidiabetic actions.

Consequently, this review aimed to discuss scientific evidence regarding a positive role of berry consumption on the prevention or delay of diabetes development and reduction or avoidance of diabetes-related complications. Moreover, a detailed composition of different berries is also presented. 


\section{Methods}

Studies examining berry consumption and T2DM were searched for (last time accessed 15 June 2020) in the Medline/PubMed, ScienceDirect/Scopus, and Web of Sciences databases using the following keywords and phrases: berry consumption and diabetes, berry polyphenolic compounds and diabetes, berry intake and glucose metabolism, berries and high-fat diets, berries and high-glycemic diets, metabolic fingerprinting of berries, lipid metabolism and berries, glycemic control, human clinical trials with berries, in vitro/in vivo studies using berries, and individual berry names. The search using these keywords and phrases resulted in more than 3000 articles in said scientific databases, as illustrated in detail in Figure 1. All articles not in line with the objectives of this review article were not considered. Additionally, the articles that were found more than once in these databases were counted once, and after removal of these duplicate and irrelevant records, nearly 2645 publications were thoroughly screened for inclusion eligibility. Finally, 336 publications were found relevant and fit to be reviewed. Only studies examining berries or berry product consumption relative to metabolic syndrome conditions or otherwise DM respective and berry fingerprinting were included. The schematic flow diagram for the selection of studies in this work is presented in Figure 1.
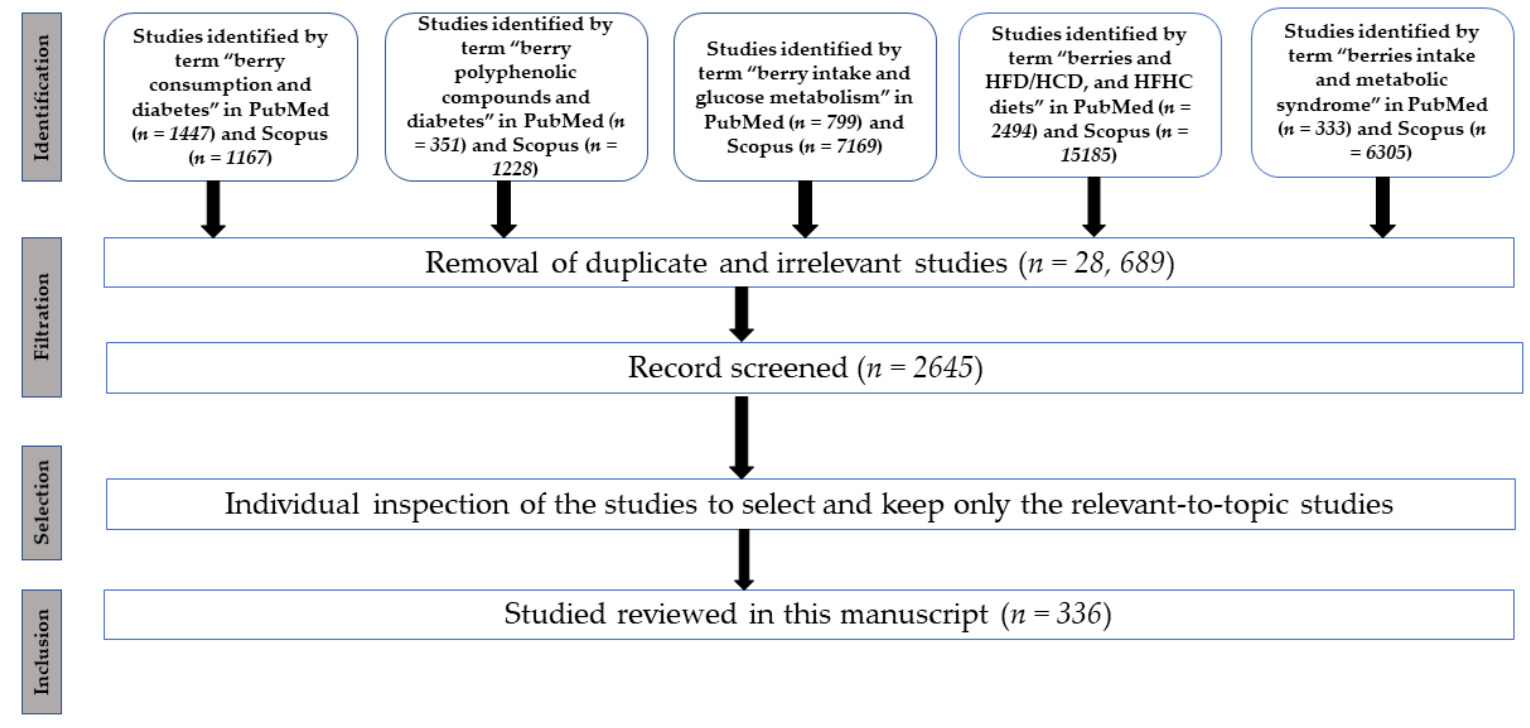

Removal of duplicate and irrelevant studies $(n=28,689)$

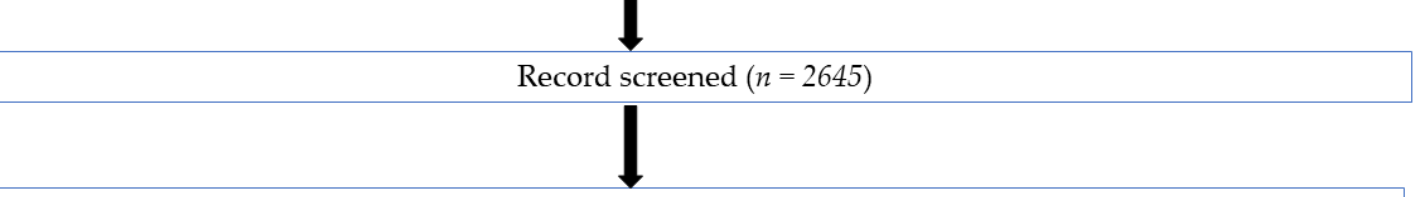

Individual inspection of the studies to select and keep only the relevant-to-topic studies

Studied reviewed in this manuscript $(n=336)$

Figure 1. Schematic representation of Preferred Reporting Items for Systematic Reviews and Meta-Analyses (PRISMA) flow diagram collection and selection of studies included in this review. Adapted from Moher, Liberati [8].

\section{Blueberries}

Blueberries (BlBs) top the list of five fruits recommended by the Food and Agriculture Organization of the United Nations (FAO) against diabetes, cancer, liver disease, anemia, and cardiovascular disease (CVD). Initially, the in vitro antidiabetic activity of BlBs were reported by Barberis et al. [9] and Martineau et al. [10]. Barberis et al. described the reduced amount of glucose absorption in the Caco-2TC7 monolayer human intestinal cell line in the supplementation of phosphate-buffered-saline (PBS) containing BlB juice (BlBJ) prior to glucose stimulation. Martineau et al. [10] used insulin-dependent/independent 3T3-L1, C2C12, and TC-tet cell lines. The overnight incubation of these cells with BlB extracts (BlBEs) enhanced glucose uptake even in the absence of insulin compared to the vehicle-delivered control cell cultures [10]. The basal secretion of insulin from TC-tet cells increased 2.5 times to 7.5 times with increasing glucose amounts from $6 \mathrm{mM}$ to $10 \mathrm{mM}$. A significant increase in glucose-stimulated insulin secretion (GSIS) was also seen after treating cells with BlBEs [10]. The BlBE adipogenic effects were also examined by assessing lipid formation and accumulation in pre-adipocytes, and BlB treatment was almost as effective as the positive control, rosiglitazone, for lipid accumulation. BlB consumption downregulated 
the HFD-induced upregulation of total cholesterol (TC), low-density lipoprotein cholesterol (LDL-C), leptin, and inflammatory genes (L-6, TNF- $\alpha$, inducible nitric oxide synthase (iNOS)), monocyte chemo-attractant protein-1 (MCP-1) (an inflammatory cytokine), peroxisome proliferator-activated receptors $\gamma$ (PPAR- $\gamma$ ), and fatty acid synthase (FAS) [11,12].

Hypoglycemic and hypolipidemic potential of BlBs or its polyphenol rich products has also been checked in many in vivo studies; Grace et al. [13] fed streptozotocin (STZ)-induced diabetic rats diets supplemented with phenolic- and anthocyanin-enriched BlBES. Anthocyanin-enriched diets increased hypoglycemic activity (51\%) compared with phenolic-enriched diets (33\%) and metformin-treated controls (32\%), suggesting that anthocyanins modulated hyperglycemic and hyperlipidemic activities [13]. The supplementation of BlBE increased the beneficial glucose metabolism involved peroxisome proliferator response element (PPRE) (1.3-1.8\%), glucose transporter 2 (GLUT-2) $(1.5 \%)$, and PPAR- $\gamma(1.4 \%)$ activities, and reduced the proinflammatory nuclear factor (NF)- $\mathrm{KB}$ activity [14]. Furthermore, an increase in the intercellular levels of the mRNA of glucose transporter (GLUT4), insulin receptor substrate-1/2 (IRS-1/IRS-2) (insulin response mediators regarding glucose metabolism), and AMP-activated protein kinase (AMPK) (a key regulator of mitochondrial biogenesis and cellular energy homeostasis) were observed in skeletal muscles, indicating increased glucose uptake $[15,16]$. BlB metabolites, especially anthocyanins, also promoted glucagon-like peptide-1 (GLP-1) expression and PPAR activity; GLP-1 increases glucose-dependent insulin secretion and pancreatic $\beta$-cell proliferation, whereas PPAR and nuclear fatty acid receptors improve IR [17]. Few studies have also shown improved insulin resistance (I) but with conflicting results in reduced BW gains [16,18,19]. However, in human clinical trials, improved insulin sensitivity without BW changes was observed [20]. Thus, insulin sensitivity may increase following BlB consumption, but BlBE may be less effective for modulating weight loss. Besides the BlBE, a few studies have also used the whole BlB fruit to determine its antidiabetic and anti-obesity potential in a group of people at high risk of T2DM (Table 1). BlB smoothie supplementation significantly reduced insulin resistance (IR) [21]. The ad libitum consumption of $100 \%$ pure BlBJ showed hypoglycemic activity, suppressing fatty acid synthase (FAS)- and $\beta$-oxidation-related gene expression in HFD-fed C57BL/6 mice (Table 1) [12]. Alcohol-free fermented juice, containing 30\% BlBJ and 70\% blackberry juice, reduced epididymal fat pad weights, percent fat mass, plasma triglyceride, and total cholesterol (TC) levels as well as mean adipocyte diameters and improved fasting blood glucose and GTT levels [22]. In another study, BlBJ consumption increased glucose uptake and inhibited adipogenesis by reducing adiponectin levels in KKKy mice [23]. In addition to BlBJ, BlB powder consumption in sugar-matched/sugar-non-matched smoothies extended the post-prandial glucose response and reduced peak postprandial glucose levels [24]. Diabetes and obesity are inter-linked via chronic inflammatory conditions, where macrophages infiltrate and accumulate in adipose tissue, triggering pro-inflammatory cytokine secretion [25]. BlB supplementation reduced these (pro)-inflammatory cytokine secretions (i.e., NF-kB, interleukin (IL)-10, tumor necrosis factor (TNF)- $\alpha$, and IL-6 expression) in obese and diabetic mice [25]. BlBE consumption also showed excellent anti-inflammatory effects against soluble vascular cell adhesion molecule-1 (sVCAM-1) (inflammatory biomarker), MCP-1, C-reactive protein (CRP) (acute inflammatory protein), and vascular endothelium [26]. 
Oxidative stress increases reactive oxygen species (ROS), chemokines, nitric oxides (NOs), adhesion molecules, nuclear factor $(\mathrm{I} \kappa \mathrm{B} \alpha)$ production, and glycation prior or after diabetes. Human aortic endothelial cells (HAECs) treated with purified BlB anthocyanins (hippuric acid, hydroxyhippuric acid, isovanillic acid-3-sulfate, benzoic acid-4-sulfate, and vanillic acid-4-sulfate) demonstrated reduced ROS, chemokine, NO, adhesion molecule, and I $\mathrm{kB} \alpha$ production [27]. In a human clinical trial, post-exercise blueberry BIB consumption decreased manganese superoxide dismutase (Mn-SOD) levels [28]. Li et al. [29] reported anti-oxidative and anti-inflammatory cytokine marker suppression by 19 and 31\%, respectively, in adipocytes and macrophages co-cultured with piceatannol, a BlB-derived bioactive compound. Piceatannol also ameliorated malfunctioning insulin-stimulated glucose uptake by upregulating Akt phosphorylation (crucial for IRS activation and hence increasing insulin sensitivity) and forkhead box O1 (FOXO1) (a transcription factor). Pterostilbene, a PPAR- $\alpha$ agonist found in BlB, promoted fatty acid catabolism by upregulating (up to 3\%) of AMPK, carnitine palmitoyl transferase-1 (CPT-1) (an enzyme for long-chain fatty acid-LCFA oxidation), acyl-CoA oxidase (ACOX) (enzyme of $\beta$-oxidation system), and uncoupling protein-2 (UCP-2) (a protein involved in glucose disposal, insulin secretion, and cellular energy dissipation) expression. AMPK is associated with mitochondrial energy production, and AMPK activation regulates liver cell gluconeogenesis by suppressing glucose-6-phosphatase (G6Pase), phosphoenolpyruvate carboxykinase (PEPCK) (gene involved in glyceroneogenesis and gluconeogenesis), FOXO1, PPAR- $\gamma$ coactivator $1 \alpha$ (PGC- $1 \alpha)$, and glucose production. FOXO1 regulates PEPCK, PGC- $1 \alpha$, and G6Pase expression, thus affecting glucose release [30].

The integration of BlB polyphenols with a HFD also attenuated HFD rendered disorders and dysbiosis. The BlB powder supplementation improved the systematic inflammation and insulin sensitivity by modulating the gut microbial population in rat fed on a HFD [31]. In human, BIB intervention offered the prebiotic-effect by increasing the relative abundance of beneficial fermentative bacterium Bifdobacterium spp [32]. The BlB-derived anthocyanins also improved the IMBD restoration by decreasing the population of E. coli [33]. More recently, Rodríguez-Daza and Daoust [34] also witnessed that BlB-derived proanthocyanidins did not significantly improve the dysbiosis symbolic Firmicutes:Bacteroidetes ratio, but its supplementation did improve the population of genera (Akkermansia, Adlercreutzia, an unknown genus of order Clostridiales, Peptostreptococcaceae, and Ruminococcaceae) considered responsible for the maintenance and restoration of the colon mucosal barrier. The health promoting role of $\mathrm{BlB}$ and its byproducts can be explained further by a comprehensive metabolite profile for BlB/BlBE [10,35-39] and is shown in Table 2. The metabolic fingerprinting reveals $\mathrm{BlB} / \mathrm{BlBE}$ as a rich source of antioxidative, antidiabetic, anti-inflammatory anthocyanins, proanthocyanidins, epicatechins, aglycons, glycosides, catechins, phenolic acids, chlorogenic acids, caffeic acid derivatives, and quercetin derivatives. Collectively, these studies demonstrated that BlB supplementation protected against HFD/HCD-induced IR hyperglycemia, pro-inflammatory responses, oxidative stress, adipocyte death, and improved insulin sensitivity, with mixed results for HFD-induced BW gain. The identified anthocyanins associated with these activities include glucosides, galactosides, and arabinosides of cyanidin, delphinidin, malvidin, peonidin, and petunidin. 
Table 1. A comprehensive list of berry interventions and their consequent effects on various levels.

\begin{tabular}{|c|c|c|c|c|c|c|c|}
\hline No. & Study Design & Study Subject & Duration & Berry Interventions & Intervention Diet & Significant Findings & Ref. \\
\hline \multicolumn{8}{|c|}{ (I) Blueberries (BlB) (Animal studies) } \\
\hline 1 & RCT & $\mathrm{C} 57 \mathrm{bl} / 6 \mathrm{~J}$ mice $(n=\mathrm{NS})$ & $12 \mathrm{wk}$ & BlB anthocyanins 300-500 mg/kg.BW.day & $\begin{array}{l}\text { LFD (20\% kcal from lard fat) } \\
\text { and HFD (70\%kcal from } \\
\text { lard fat) }\end{array}$ & $\begin{array}{l}\text { Lower the blood glucose level and } \\
\text { dyslipidemia markers }\end{array}$ & [13] \\
\hline 2 & RCT & Male C57BL/6 mice $(n=24)$ & $8 \mathrm{wk}$ & $\begin{array}{l}4 \%(w / w) \text { F/D whole BlB powder with HFD } \\
\text { provided ad libitum }\end{array}$ & $\operatorname{HFD}(60 \%$ kcal from fat $)$ & $\begin{array}{l}\text { Offer protection against HFD-induced } \\
\text { obesity, adipose tissue macrophages } \\
\text { inflammatory gene expression, } \\
\text { and oxidative stress }\end{array}$ & [11] \\
\hline 3 & RCT & C57/B16 mice $(n=200)$ & $12 \mathrm{wk}$ & $\begin{array}{c}5 \% \text { or } 10 \%(w / w) \text { of whole BlB with HFD } \\
\text { provided ad libitum }\end{array}$ & $\operatorname{HFD}(45 \%$ kcal from fat) & $\begin{array}{l}\text { Reduced HFD-induced cellular } \\
\text { inflammatory cytokines, chemokines, } \\
\text { interleukins, and proinflammatory } \\
\text { interferon gamma -producing T-cells }\end{array}$ & [18] \\
\hline 4 & RCT & $\begin{array}{l}\text { Male Zucker Fatty and Zucker } \\
\text { Lean rats }(n=48)\end{array}$ & $8 \mathrm{wk}$ & $\begin{array}{l}4 \%(w / w) \text { F/D whole BlB powder with HFD } \\
\text { provided ad libitum }\end{array}$ & $\operatorname{HFD}(45 \%$ kcal from fat) & $\begin{array}{l}\text { Hypolipidemic, Hyper-insulinemic, } \\
\text { hypoglycemic and anti-inflammatory }\end{array}$ & [40] \\
\hline 5 & RCT & C57BL/6 mice $(n=48)$ & $12 \mathrm{wk}$ & $\begin{array}{l}\text { Ad libitum } 100 \% \text { BlBJ with HFD provided } \\
\text { ad libitum }\end{array}$ & $\begin{array}{l}\text { LFD ( } 20 \% \text { kcal from fat }) \text { and } \\
\text { HFD ( } 45 \% \text { kcal from fat })\end{array}$ & $\begin{array}{c}\text { Reduced expressions of inflammatory } \\
\text { and FA synthesis genes. Reduced IR and } \\
\text { plasma dyslipidemia markers }\end{array}$ & [12] \\
\hline 6 & RCT & C57BL/6 mice $(n=72)$ & $8 \mathrm{wk}$ & $\begin{array}{c}65.1 \pm 1.6 \mathrm{mg} \text { cyanidin-3-O-glucoside } / \mathrm{L} \\
\text { (from } 30 \% \text { BlB }+70 \% \text { blackberry juice } \\
\text { available ad libitum) }\end{array}$ & $\operatorname{HFD}(60 \%$ kcal from fat $)$ & Anti-obesity, hypoglycemic, antidiabetic & [22] \\
\hline 7 & RCT & $\begin{array}{l}\text { C57BL/6 and KKAy mice } \\
\qquad(n=20)\end{array}$ & $4 \mathrm{wk}$ & $\begin{array}{l}\text { BlBJ }(40-80 \mathrm{~mL} / \mathrm{kg} \text { per day in } \\
\text { drinking water) }\end{array}$ & Normal chow diet & $\begin{array}{l}\text { Improved glucose tolerance, reduced } \\
\text { glycemic response suggesting increased } \\
\text { insulin sensitivity }\end{array}$ & [23] \\
\hline 8 & RCT & Obese Zucker rat $(n=20)$ & $8 \mathrm{wk}$ & $8 \%$ wild BlB diet (WB) provided ad libitum & NA & $\begin{array}{l}\text { Downregulated expression and plasma } \\
\text { concentrations of NF-kB, TNF } \alpha \text {, IL- } 6 \text {, } \\
\text { CRP in liver and adipose tissues }\end{array}$ & [25] \\
\hline \multicolumn{8}{|c|}{ Human studies } \\
\hline 9 & $\mathrm{SB}$ and RCT & $\begin{array}{l}\text { Obese men and women } \\
\qquad(n=66)\end{array}$ & $8 \mathrm{wk}$ & $50 \mathrm{~g} \mathrm{~F} / \mathrm{DB} 1 \mathrm{~B}$ per day & NA & $\begin{array}{l}\text { Reduction in plasma oxidized LDL and } \\
\text { other plasma lipid oxidation products }\end{array}$ & [20] \\
\hline 10 & $\mathrm{DB}, \mathrm{PC}, \mathrm{RCT}$ & $\begin{array}{l}\text { overweight or obese } \\
\text { individuals }(n=30)\end{array}$ & $4 \mathrm{wk}$ & $\begin{array}{l}4 \mathrm{~g} \text { of inulin/day from BlB (equivalent to } \\
\text { two cups of whole BlB) }\end{array}$ & NA & $\begin{array}{l}\text { Improvement in glycemic response, } \\
\text { insulin sensitivity, satiety, serum lipid } \\
\text { parameters, and fecal markers of } \\
\text { gut microbiota }\end{array}$ & [41] \\
\hline 11 & $\mathrm{DB}, \mathrm{PC}, \mathrm{RCT}$ & Diabetic patients $(n=58)$ & $24 \mathrm{wk}$ & $160 \mathrm{mg}$ of BlB anthocyanins twice daily & NA & $\begin{array}{l}\text { Reduced serum concentration of LDL-C, } \\
\text { TG, apolipoprotein, apolipoprotein C-III, } \\
\text { lipid and protein oxidation markers with } \\
\text { strengthening the inherent } \\
\text { antioxidative system }\end{array}$ & [42] \\
\hline
\end{tabular}


Table 1. Cont.

\begin{tabular}{|c|c|c|c|c|c|c|c|}
\hline No. & Study Design & Study Subject & Duration & Berry Interventions & Intervention Diet & Significant Findings & Ref. \\
\hline 12 & DB, PC, RCT & Healthy adults $(n=44)$ & $6 \mathrm{wk}$ & 45 g/day F/D BlBpowder & $\begin{array}{l}\text { 12-oz yogurt and skim } \\
\text { milk-based smoothie }\end{array}$ & $\begin{array}{l}\text { Improvement in endothelial function in } \\
\text { subjects with metabolic syndrome }\end{array}$ & [21] \\
\hline 13 & $\mathrm{DB}, \mathrm{CO}, \mathrm{RCT}$, & Healthy human beings $(n=17)$ & $4 \mathrm{wk}$ & 310-724 mg/kg.BW.day BlBanthocyanin & Sugar-matched smoothie & $\begin{array}{c}\text { Extend the postprandial glucose } \\
\text { response beyond the period observed for } \\
\text { a sugar-matched control }\end{array}$ & [24] \\
\hline \multicolumn{8}{|c|}{ (I) Bilberries (BB) (Animal studies) } \\
\hline 1 & RCT & Male KK-Ay mice $(n=16)$ & $5 \mathrm{wk}$ & $27 \mathrm{~g}$ of $\mathrm{BB}$ extract $/ \mathrm{kg}$ diet daily & NA & $\begin{array}{l}\text { Activation of AMP-activated protein } \\
\text { kinase (AMPK) resulting in increased } \\
\text { insulin sensitivity, upregulation of } \\
\text { glucose transporter GLUT4, suppression } \\
\text { of glucose production in liver }\end{array}$ & [15] \\
\hline 2 & RCT & $\begin{array}{l}\text { diabetic groups of Wistar rats } \\
\qquad(n=32)\end{array}$ & $4 \mathrm{wk}$ & BB extracts $2 \mathrm{~g} /$ day by gavage & Normal chow diet & $\begin{array}{l}\text { Increased serum insulin, reduced TC, } \\
\text { VLDL-C, LDL-C, and TG levels, } \\
\text { and prevented HDL-C decline }\end{array}$ & {$[17,43]$} \\
\hline 3 & $\mathrm{RCT}$ & $\begin{array}{l}\text { Brown Norway (BN) rats } \\
\qquad(n=96)\end{array}$ & $6 \mathrm{wk}$ & BB extract $100 \mathrm{mg} / \mathrm{kg}$. BW.day & Normal chow diet & Prevent diabetic retinopathy & [44] \\
\hline 4 & RCT & Male KM mice $(n=60)$ & $5 \mathrm{~d}$ & $\begin{array}{c}\text { BB extract (containing } 42.04 \% \\
\text { anthocyanins) } 200 \mathrm{mg} / \mathrm{kg} \text { BW·day) }\end{array}$ & Normal chow diet & $\begin{array}{l}\text { Reduced the live damage and oxidative } \\
\text { stress markers (ALT, MDA, NO) with } \\
\text { improvements in enzymatic } \\
\text { antioxidative system (GSH) }\end{array}$ & [45] \\
\hline 5 & RCT & Male Wistar rats $(n=15)$ & $12 \mathrm{wk}$ & $\begin{array}{l}40 \mathrm{mg} / \mathrm{kg} \text {.day BB extracts in } 5 \mathrm{~mL} \\
\text { drinking water }\end{array}$ & Normal chow diet & Prevent capillary albumin filtration & [46] \\
\hline 6 & RCT & $\begin{array}{c}\text { Goto-Kakizaki (GK) rat } \\
(n=\mathrm{NS})\end{array}$ & $4 \mathrm{wk}$ & BB decoction with rodent chow & powdered rodent chow & $\begin{array}{l}\text { Improved mitochondrial respiratory and } \\
\text { biogenesis parameters }\end{array}$ & [47] \\
\hline \multicolumn{8}{|c|}{ (Human studies) } \\
\hline 7 & $\mathrm{DB}, \mathrm{CO}, \mathrm{RCT}$ & $\begin{array}{l}\text { T2DM Male volunteer subjects } \\
\qquad(n=8)\end{array}$ & $24 \mathrm{~h}$ & $\begin{array}{l}\text { A single capsule of } 0 \times 47 \mathrm{~g} \text { BB extract } \\
(36 \% w / w)\end{array}$ & NA & $\begin{array}{l}\text { Decrease in the incremental AUC for } \\
\text { both glucose and insulin without } \\
\text { alterations in GLP1, glucagon, amylin, } \\
\text { and anti-inflammatory peptides }\end{array}$ & [48] \\
\hline 8 & $\begin{array}{l}\mathrm{CO}, \mathrm{DB}, \mathrm{RCT} \\
\text { design }\end{array}$ & $\begin{array}{l}\text { Obese/Overweight/diabetic } \\
\text { men and women }(n=16)\end{array}$ & $3 \mathrm{wk}$ & $\begin{array}{c}3 \times 0.47 \mathrm{~g} \text { of Mirtoselect capsules per day } \\
\text { a standardized BB extract }(36 \% w / w) \\
\text { anthocyanins) }\end{array}$ & NA & $\begin{array}{l}\text { Reduced activity of digestion enzymes } \\
\text { without alterations in anti-inflammatory } \\
\text { markers, vascular health markers and } \\
\text { reducing capacity }\end{array}$ & [49] \\
\hline 9 & $\mathrm{RCT}$ & $\begin{array}{l}\text { Healthy men and women } \\
\qquad(n=9)\end{array}$ & $1 \mathrm{~d}$ & $10 \% \mathrm{BB}$ in fermented drink up to $300 \mathrm{~g} /$ day & White bread & $\begin{array}{l}\text { Lower the insulin response than } \\
\text { glycemic response }\end{array}$ & [50] \\
\hline 10 & RCT & $\begin{array}{l}\text { Healthy men and women } \\
\qquad(n=62)\end{array}$ & $4 \mathrm{wk}$ & BB juice $330 \mathrm{~mL} /$ day & NA & Anti-inflammatory & [51] \\
\hline
\end{tabular}


Table 1. Cont.

\begin{tabular}{|c|c|c|c|c|c|c|c|}
\hline No. & Study Design & Study Subject & Duration & Berry Interventions & Intervention Diet & Significant Findings & Ref. \\
\hline 11 & Parallel RCT & Healthy men $(n=40)$ & $8 \mathrm{wk}$ & Fresh BB $100 \mathrm{~g} /$ day of BB & NA & $\begin{array}{l}\text { Increased intestinal bioavailability of } \\
\text { antioxidative and } \\
\text { antidiabetic compounds }\end{array}$ & [52] \\
\hline 12 & $\mathrm{RCT}$ & $\begin{array}{l}\text { Healthy men and women } \\
\qquad(n=27)\end{array}$ & $8 \mathrm{wk}$ & Fresh BB $400 \mathrm{~g} /$ day & NA & $\begin{array}{l}\text { Reduction in the low-grade } \\
\text { inflammation with different cytoplasmic } \\
\text { ribosomal proteins, Toll-like receptor, } \\
\text { and B-cell receptor signaling pathways }\end{array}$ & [53] \\
\hline \multicolumn{8}{|c|}{ (I) Cranberries (CrB) (Animal studies) } \\
\hline 1 & RCT & 36 C57Bl/6J male mice & $8 \mathrm{wk}$ & $\begin{array}{l}\mathrm{CrB} \text { extracts } 200 \mathrm{mg} / \mathrm{kg} \mathrm{BW} \text { on daily basis } \\
\text { by gavage }\end{array}$ & $\begin{array}{l}\text { HFD ( } 65 \% \text { lipids, } 15 \% \text { proteins } \\
\text { and } 20 \% \text { carbohydrates) }\end{array}$ & $\begin{array}{c}\text { Attenuated HFD-induced obesity, TC } \\
\text { and TG accumulation, oxidative stress, } \\
\text { with improvements in glycemic response, } \\
\text { insulin sensitivity, HOMAIR, alleviate } \\
\text { intestinal inflammation }\end{array}$ & [54] \\
\hline 2 & $\mathrm{RCT}$ & Male Fischer rats $(n=24)$ & $16 \mathrm{~m}$ & $\begin{array}{l}2 \% \text { whole CrB powder standard NIH-31 } \\
\text { standard rodent chow available ad libitum }\end{array}$ & NA & $\begin{array}{l}\text { Increased } \beta \text {-cell glucose responsiveness; } \\
\text { age related decline in in basal plasma } \\
\text { insulin concentrations was delayed } \\
\text { by cranberry }\end{array}$ & [55] \\
\hline \multicolumn{8}{|c|}{ Human studies } \\
\hline 3 & $\mathrm{CO}, \mathrm{RCT}$ & $\begin{array}{l}\text { Obese participants } \\
\qquad(n=25)\end{array}$ & $2-4 \mathrm{~h}$ & Cranberries (40 g) & HF breakfast (70 g fat, $974 \mathrm{kcal})$ & $\begin{array}{l}\text { Improved postprandial glycemic control, } \\
\text { reduction in lipid oxidation products and } \\
\text { inflammatory cytokines }\end{array}$ & [56] \\
\hline 4 & $\mathrm{PC}, \mathrm{DB}, \mathrm{RCT}$ & $\begin{array}{l}\text { T2DM men and women } \\
\qquad(n=30)\end{array}$ & $12 \mathrm{wk}$ & $\begin{array}{l}3 \text { capsules of } \mathrm{CrB} \text { extract/day } \\
(1 \text { capsule }=500 \mathrm{mg})\end{array}$ & NA & Decrease in the TC:HDLC ratio & [57] \\
\hline 5 & single CO RCT & $\begin{array}{l}\text { Healthy men and women } \\
\qquad(n=12)\end{array}$ & OTCS & $\begin{array}{l}\text { Dextrose sweetened normal calorie CrB } \\
\text { juice (NCCBJ; } 27 \% \text { CBJ, v/v; } \\
130 \mathrm{Cal} / 240 \mathrm{~mL} \text { ) and low-calorie CrB juice } \\
\text { (LCCBJ; } 27 \% \text {, v/v CrBJ;19 Cal/240 mL) }\end{array}$ & $\begin{array}{l}5 \mathrm{~g} \text { Vanilla Crisp Power Bar } \\
\text { (contained } 230 \text { Cal, } 2.5 \mathrm{~g} \text { total } \\
\text { fat, } 3 \mathrm{~g} \text { dietary fiber, } 20 \mathrm{~g} \text { sugars, } \\
22 \mathrm{~g} \text { other carbo-hydrates, } \\
\text { and } 9 \mathrm{~g} \text { protein }\end{array}$ & $\begin{array}{l}\text { Improved metabolic response } \\
\text { towards glucose }\end{array}$ & [58] \\
\hline 6 & RCT & $\begin{array}{l}\text { Non-diabetic men and women } \\
\qquad(n=187)\end{array}$ & OTCS & $\begin{array}{c}\text { low-calorie } 27 \% \mathrm{CrBJ}(19 \mathrm{Cal} / 240 \mathrm{~mL}) \\
\text { normal-calorie } 27 \% \mathrm{CrBJ}(140 \mathrm{Cal} / 240 \mathrm{~mL}) \\
\text { at weight-adjusted serving size } \\
(480 \mathrm{~mL} / 70 \mathrm{~kg})\end{array}$ & NA & Improved glycemic response & [59] \\
\hline 7 & RCT & $\begin{array}{l}\text { T2DM men and women } \\
\qquad(n=13)\end{array}$ & OTCS & $\begin{array}{l}\text { Raw CrB ( } 55 \mathrm{~g}, 21 \mathrm{cal}, 1 \mathrm{~g} \text { fiber); Sweetened } \\
\text { dried CrB ( } 40 \mathrm{~g}, 138 \mathrm{cal}, 2.1 \mathrm{~g} \text { fiber); } \\
\text { Sweetened dried CrB containing less sugar } \\
\text { (SDC-LS, } 40 \mathrm{~g}, 113 \text { cal, } 1.8 \mathrm{~g} \text { fiber }+10 \mathrm{~g} \\
\text { polydextrose) }\end{array}$ & $\begin{array}{l}\text { White bread ( } 57 \mathrm{~g}, 160 \mathrm{cal} \text {, } \\
1 \mathrm{~g} \text { fiber) }\end{array}$ & $\begin{array}{l}\text { favorable glycemic and } \\
\text { insulinemic response }\end{array}$ & [60] \\
\hline
\end{tabular}


Table 1. Cont.

\begin{tabular}{|c|c|c|c|c|c|c|c|}
\hline No. & Study Design & Study Subject & Duration & Berry Interventions & Intervention Diet & Significant Findings & Ref. \\
\hline 8 & $\begin{array}{l}\text { CS Nutrition } \\
\text { Examination } \\
\quad \text { Survey } \\
(n=10891) \\
\end{array}$ & Healthy men and women & 2 days & Average 2-day CrBJ intake 158 to $404 \mathrm{~mL}$ & Routine diet & $\begin{array}{l}\text { Lowered the weight-gain, TC, and } \\
\text { proinflammatory serum CRP levels }\end{array}$ & [61] \\
\hline 9 & $\mathrm{DB}, \mathrm{CO}, \mathrm{RCT}$ & $\begin{array}{l}\text { Healthy men and women } \\
\qquad(n=12)\end{array}$ & OTCS & $\begin{array}{c}37.5 \mathrm{~g} \text { of } \mathrm{CrB} \text { in addition to } 37.5 \mathrm{~g} \times 3 \text { of } \\
\text { other berries (bilberries, strawberries, } \\
\text { blueberries) }+35 \mathrm{~g} \text { added sugar }\end{array}$ & NA & Hypoglycemic and hypo-insulinemic & [62] \\
\hline 10 & Parallel RCT & $\begin{array}{l}\text { Diabetic men and women } \\
\qquad(n=48)\end{array}$ & $8 \mathrm{wk}$ & $\begin{array}{c}200 \times 2 \mathrm{~mL} \text { RCCJ was enriched with } \\
\text { omega- } 3 \text { fatty acid ( } 180 \mathrm{mg} \text { EPA }+120 \mathrm{mg} \\
\text { DHA) on daily basis }\end{array}$ & $\begin{array}{l}\text { usual diet and physical activity } \\
\text { during the study }\end{array}$ & Anti-dyslipidemic and hypoglycemic & [63] \\
\hline 11 & Parallel DB RCT & T2DM male patients $(n=58)$ & $12 \mathrm{wk}$ & 1 cup $(240 \mathrm{~mL})$ CrB juice daily & NA & $\begin{array}{l}\text { Antiglycation, antidiabetic, reducing } \\
\text { CVD risk factors in T2DM male patients }\end{array}$ & [64] \\
\hline 12 & $\begin{array}{c}\text { Parallel DB, PC } \\
\text { RCT }\end{array}$ & $\begin{array}{l}\text { Healthy men and women } \\
\qquad(n=56)\end{array}$ & $8 \mathrm{wk}$ & $480 \mathrm{~mL}$ (80 kcal) whole CrB juice daily & $\begin{array}{l}\text { Complete diets in addition to } \\
\text { Background diets consisted of } \\
\text { typical American foods (HFD) } \\
\text { and 3-5 servings of fruits or } \\
\text { vegetables daily ( } 328-618 \mathrm{~g} / \mathrm{d} \\
\text { depending on energy intake) }\end{array}$ & $\begin{array}{l}\text { Anti-dyslipidemic, hypoglycemic, } \\
\text { improved HOAM-IR }\end{array}$ & [65] \\
\hline 13 & RCT & $\begin{array}{l}\text { Patients with metabolic } \\
\text { syndrome }(n=55)\end{array}$ & $60 \mathrm{~d}$ & $\begin{array}{l}0.7 \mathrm{~L} / \text { day }(\mathrm{J}(20 \mathrm{kcal}) \text { of reduced-energy } \mathrm{CrB} \\
\text { juice containing } 66 \mathrm{mg} \text { proanthocyanidins; } \\
\text { total phenolics of } 104 \text { and } 0 \cdot 12 \mathrm{mg} \text { folic acid }\end{array}$ & NA & $\begin{array}{l}\text { An increase in adiponectin and folic acid } \\
\text { and a decrease in homocysteine, } \\
\text { decreased lipoperoxidation and protein } \\
\text { oxidation levels }\end{array}$ & [66] \\
\hline 14 & $\mathrm{CO}, \mathrm{DB}$ & Obese yet healthy men $(n=35)$ & $4 \mathrm{wk}$ & $500 \mathrm{~mL}$ CrB juice/day & NA & Improved augmentation in obese men & [67] \\
\hline 15 & $\mathrm{DB}, \mathrm{CO}$ & Obese men $(n=30)$ & $12 \mathrm{wk}$ & $\begin{array}{l}\text { Increasing doses of low-calories CrBJ } \\
\text { during three successive periods of } 4 \text { wk } \\
\text { (wk 1-4: } 125 \mathrm{~mL} / \text { day, wk 5-8: } 250 \mathrm{~mL} / \text { day, } \\
\text { and wk 9-12: } 500 \mathrm{~mL} / \text { day) }\end{array}$ & NA & Improved antioxidative defense system & [68] \\
\hline 16 & $\mathrm{DB}, \mathrm{CO}$ & Obese men $(n=30)$ & $12 \mathrm{wk}$ & $\begin{array}{l}\text { Increasing doses of low-calories CrBJ } \\
\text { during three successive periods of } 4 \text { wk } \\
\text { (wk 1-4: } 125 \mathrm{~mL} / \text { day, wk 5-8: } 250 \mathrm{~mL} / \text { day, } \\
\text { and wk 9-12: } 500 \mathrm{~mL} / \text { day) }\end{array}$ & NA & $\begin{array}{c}\text { Decrease in plasma OxLDL, intercellular } \\
\text { adhesion molecule- } 1 \text { (ICAM-1), vascular } \\
\text { cell adhesion molecule-1 (VCAM-1) and } \\
\text { E-selectin concentrations }\end{array}$ & [69] \\
\hline 17 & $\mathrm{CO}, \mathrm{DB}$ & $\begin{array}{l}\text { Abdominally obese men } \\
\qquad(n=30)\end{array}$ & $12 \mathrm{wk}$ & $\begin{array}{l}\text { Increasing doses of low-calories CrBJ } \\
\text { during three successive periods of } 4 \text { wk } \\
\text { (wk 1-4: } 125 \mathrm{~mL} / \text { day, wk 5-8: } 250 \mathrm{~mL} / \text { day, } \\
\text { and wk 9-12: } 500 \mathrm{~mL} / \text { day) }\end{array}$ & NA & $\begin{array}{l}\text { Increased plasma HDL-cholesterol } \\
\text { concentrations }\end{array}$ & [70] \\
\hline 18 & RCT & Healthy men $(n=21)$ & $2 \mathrm{wk}$ & CrBJ 7 mL/kg.BW.day & NA & $\begin{array}{l}\text { Reduction in plasma OxLDL levels and } \\
\text { Improved antioxidative defense system }\end{array}$ & [71] \\
\hline
\end{tabular}


Table 1. Cont.

\begin{tabular}{|c|c|c|c|c|c|c|c|}
\hline No. & Study Design & Study Subject & Duration & Berry Interventions & Intervention Diet & Significant Findings & Ref \\
\hline \multicolumn{8}{|c|}{ (I) Raspberries (RB) (Animal studies) } \\
\hline 1 & RCT & $\begin{array}{l}\text { Weanling male Syrian } \\
\text { golden hamsters }\end{array}$ & $12 \mathrm{wk}$ & RBJ $275 \mathrm{~mL} /$ day $(1 \mathrm{~mL}=0.6 \mathrm{~g}$ of berries $)$ & $\begin{array}{l}\text { semi-purified hyperlipidic diet } \\
\text { (0.5\% cholesterol and } 15 \% \text { lard })\end{array}$ & Hypo-cholestrolemic and antioxidative & [72] \\
\hline 2 & $\mathrm{RCT}$ & Male Wistar rats $(n=30)$ & $10 \mathrm{~d}$ & $\begin{array}{l}\text { Dose of ellagitannins enriched RB extracts } \\
\text { equivalent to daily consumption of } 125 \mathrm{~g} \\
\text { of fresh fruit by a human healthy adult of } \\
70 \mathrm{~kg} \text { (i.e., } 20 \mathrm{mg} / \mathrm{kg} \text { BW.day orally) }\end{array}$ & NA & $\begin{array}{l}\text { Protection from the ethanol induced } \\
\text { oxidative stress and } \\
\text { inflammatory biomarkers }\end{array}$ & [73] \\
\hline 3 & RCT & male Lewis rats $(n=24)$ & $30 \mathrm{~d}$ & RB extracts at $30-120 \mathrm{mg} / \mathrm{kg} . \mathrm{BW}$ & NA & $\begin{array}{l}\text { Inhibition of inflammation, pannus } \\
\text { formation, cartilage damage, } \\
\text { and bone resorption }\end{array}$ & [74] \\
\hline 4 & RCT & CD1 male mice $(n=36)$ & $4 \mathrm{wk}$ & RB infusion by gavage (100 mg/kg BW.day) & NA & Improved antioxidative defense system & [75] \\
\hline 5 & $\mathrm{RCT}$ & $\begin{array}{l}\text { obese diabetic }(\mathrm{db} / \mathrm{db}) \text { mice } \\
\qquad(n=30)\end{array}$ & $8 \mathrm{wk}$ & $\begin{array}{c}5.3 \% \text { RB supplementation along } \\
\text { agar-based diet finally containing } \\
\text { polyphenolics ( } 963 \mathrm{mg} \text { extractable GAE/kg } \\
\text { agar-based diet) }\end{array}$ & agar-based diet & $\begin{array}{l}\text { Hyper-cholestrolemic and } \\
\text { diabetes-induced oxidative stress }\end{array}$ & [76] \\
\hline 6 & RCT & $\begin{array}{l}\text { Male Zucker Fatty rats } \\
\qquad(n=\mathrm{NS})\end{array}$ & $12 \mathrm{wk}$ & $\begin{array}{l}20 \mathrm{~g} \text { of diet per day containing } \mathrm{RB}(2 \% \text { red } \\
\text { raspberry } \mathrm{F} / \mathrm{D} \text { powder })\end{array}$ & NA & $\begin{array}{l}\text { Upregulation of the expression of } \\
\text { myocardial adiponectin receptor } 1 \text { and } \\
\text { apolipoprotein E, improving the plasma } \\
\text { cholesterol and triglyceride homeostasis }\end{array}$ & [77] \\
\hline 7 & RCT & Male Wistar rats $(n=42)$ & $5 d$ & $\begin{array}{c}313 \mathrm{~g} \text { whole RB with/without Lactobacillus } \\
\left.\text { plantarum HEAL19 (HEAL19 at } 10^{9} \mathrm{cfu}\right) \\
\text { per day with diet }\end{array}$ & Normal chow diet & $\begin{array}{l}\text { Increased intestinal SCFA load and } \\
\text { anti-inflammatory }\end{array}$ & [78] \\
\hline 8 & RCT & Male F-344 rats $(n=\mathrm{NS})$ & $6 \mathrm{wk}$ & $\begin{array}{l}\text { AIN-76A diet containing either } 5 \% \text { whole } \\
\text { BRB powder, } 0.2 \% \\
\text { BRB anthocyanins, or } 2.25 \% \text { of the residue } \\
\text { fraction provided ad libitum }\end{array}$ & NA & $\begin{array}{l}\text { Anti-dysbiosis, anti-inflammatory, } \\
\text { anti-obesity }\end{array}$ & [79] \\
\hline 9 & RCT & $\begin{array}{c}\text { Male db/db mice with } \\
\text { C57BL/6J Background }(n=48)\end{array}$ & $8 \mathrm{wk}$ & $\begin{array}{c}150 \mathrm{mg} / \mathrm{kg} . \text { BW.day per mice RB derived } \\
\text { pelargonidin-3-O-glucoside }\end{array}$ & NA & $\begin{array}{l}\text { Hypoglycemic, anti-inflammatory, } \\
\text { anti-obesity }\end{array}$ & [80] \\
\hline 10 & RCT & $\begin{array}{l}\text { Specific-pathogen free C57BL/6 } \\
\text { mice }(n=20)\end{array}$ & $7 \mathrm{wk}$ & $\begin{array}{l}\text { AIN-76A diet with } 10 \% \text { black raspberry } \\
\text { powder provided ad libitum }\end{array}$ & NA & $\begin{array}{l}\text { Hypo-glycemic, anti-metabolic } \\
\text { syndromic }\end{array}$ & [81] \\
\hline 11 & RCT & Male $\mathrm{db} / \mathrm{db}$ mice $(n=30)$ & $8 \mathrm{wk}$ & $10 \% \mathrm{~F} / \mathrm{D} \mathrm{RB}$ in a isocaloric standard diet & Isocaloric standard diet & $\begin{array}{l}\text { Hypo-cholestrolemic, antioxidative, } \\
\text { improved insulin sensitivity }\end{array}$ & [82] \\
\hline 12 & $\mathrm{RCT}$ & C57BL/6J mice $(n=\mathrm{NS})$ & $10 \mathrm{wk}$ & $\begin{array}{l}\text { Energy-containing RB foods (juice and } \\
\text { puree concentrate and whole fruit powder) } \\
\text { containing } 10 \% \text { raspberry and HFD } \\
\text { supplemented with } 0.2 \%(w / w) \text { RB extract } \\
\text { provided ad libitum }\end{array}$ & $\begin{array}{l}\text { HFD ( } 45 \% \text { energy from fat) + } \\
\text { high-carbohydrate food ( } 35 \% \\
\quad \text { energy from starch) }\end{array}$ & Anti-obesity and antidiabetic & [83] \\
\hline
\end{tabular}


Table 1. Cont.

\begin{tabular}{|c|c|c|c|c|c|c|c|}
\hline No. & Study Design & Study Subject & Duration & Berry Interventions & Intervention Diet & Significant Findings & Ref. \\
\hline 13 & RCT & $\begin{array}{l}\text { C57BL/6J, C57BL/Ks db/db, } \\
\text { and } \mathrm{db} /+ \text { male mice }(n=\mathrm{NS})\end{array}$ & $8 \mathrm{wk}$ & 0.2\% Cyanidin 3-glucoside in HFD & $\begin{array}{l}\text { HFD ( } 58 \% \text { of calories from } \\
\text { coconut hydrogenated fat) }\end{array}$ & $\begin{array}{l}\text { Anti-obesity, anti-inflammatory, } \\
\text { improvement in the insulin sensitivity }\end{array}$ & [84] \\
\hline 14 & RCT & $\begin{array}{l}\text { Male Sprague Dawley rats } \\
\qquad(n=40)\end{array}$ & $8 \mathrm{wk}$ & $\begin{array}{c}\text { Application of RB derived EA } \\
(0.1-10 \mathrm{mg} / \mathrm{mL}) \text { on ischemic stomach } \\
(1.5 \mathrm{~mL} / 100 \mathrm{~g} . \mathrm{BW}) \text { in an in an ex } \\
\text { vivo chamber }\end{array}$ & NA & $\begin{array}{l}\text { Gastric protective action against gastric } \\
\text { lesions induced by } \mathrm{NH}_{4} \mathrm{OH} \text {, due to } \\
\text { anti-oxidative activity of EA }\end{array}$ & [85] \\
\hline 15 & RCT & Male Wistar rats $(n=22)$ & $4 \mathrm{wk}$ & $\begin{array}{c}\text { Oral administration of } 10-20 \mathrm{mg} / \mathrm{kg} . \mathrm{BW} \text { of } \\
\text { RB derived elagic acid }\end{array}$ & NA & Anti-inflammatory and anti-oxidative & [86] \\
\hline 16 & RCT & $\begin{array}{l}\text { Male Wistar rats AMPK } \alpha 1-/- \\
\qquad(n=12)\end{array}$ & $10 \mathrm{wk}$ & $\begin{array}{l}5 \% \text { supplementation of RB extracts } \\
\text { (contains polyphenols at } \sim 11 \mathrm{~g} \text { gallic acid } \\
\text { equivalent (GAE)/kg of DW) along HFD }\end{array}$ & HFD ( $60 \%$ from fat $)$ & $\begin{array}{l}\text { reduced ectopic lipid storage, alleviated } \\
\text { inflammation responses, improved } \\
\text { whole-body insulin sensitivity, and } \\
\text { promoted mitochondrial biogenesis }\end{array}$ & [87] \\
\hline 17 & RCT & Male mice $(\mathrm{C} 57 \mathrm{BL} / 6)(n=40)$ & $12 \mathrm{wk}$ & $\begin{array}{l}\text { 5\% F/D RB powder in HFD provided } \\
\text { ad libitum }\end{array}$ & $\operatorname{HFD}(60 \%$ energy from fat $)$ & Anti-dyslipidemic, hypoglycemic & [88] \\
\hline 18 & RCT & Male KK-Ay mice ( $n=$ NS) & 5 weeks & $\begin{array}{l}\text { Cyanidin 3-glucoside } 2 \mathrm{~g} / \mathrm{kg} \text {.BW.day in the } \\
\text { normal chow diet }\end{array}$ & NA & $\begin{array}{l}\text { Anti-obesity, anti-inflammatory, } \\
\text { improvement in the insulin sensitivity }\end{array}$ & [89] \\
\hline 19 & RCT & Male mice $(\mathrm{C} 57 \mathrm{BL} / 6)(n=40)$ & $12 \mathrm{wk}$ & $\begin{array}{c}\text { 3\% RB seed floor (equivalent to } 0.03 \% \\
\text { ellagic acid) in HFD and HFD + } \\
\text { High-sucrose diet }\end{array}$ & $\begin{array}{l}\text { HFD ( } 41 \% \text { energy from fat) } \\
\text { HFD + High-sucrose diet } \\
\text { (37\% energy from sucrose) }\end{array}$ & $\begin{array}{c}\text { Anti-dyslipidemic, } \\
\text { hypoglycemic, attenuated } \\
\text { hepatic ER and oxidative stresses, as well } \\
\text { as adipocyte inflammation }\end{array}$ & [90] \\
\hline \multicolumn{8}{|c|}{$\begin{array}{l}\text { Human studies } \\
\end{array}$} \\
\hline 20 & $\mathrm{PC}, \mathrm{CO}, \mathrm{RCT}$ & $\begin{array}{l}\text { Healthy men and women } \\
\qquad(n=20)\end{array}$ & $4 \mathrm{wk}$ & $\begin{array}{c}\text { High-carbohydrate bars (120-123 g) } \\
\text { containing freeze-dried black RB } \\
\text { (10\% (LOW-Rasp) or 20\% (HIGH-Rasp)), } \\
\text { One bar each day after overnight fasting. }\end{array}$ & $\begin{array}{c}\text { macronutrient-matched } \\
\text { high-carbohydrate cereal bars } \\
\text { (45\% total sugars) }\end{array}$ & & [91] \\
\hline 21 & RCT & $\begin{array}{l}\text { Healthy men and women } \\
\qquad(n=12)\end{array}$ & NS & $100 \mathrm{~g}$ RB along the designated diet & $\begin{array}{l}\text { High-carbohydrate food in the } \\
\text { form of pancakes ( } 50 \mathrm{~g} \text { available } \\
\text { carbohydrate from } \\
333 \text { kcal pancake) }\end{array}$ & $\begin{array}{l}\text { Alter postprandial hyperglycemia to } \\
\text { sustainable glycemic response }\end{array}$ & [92] \\
\hline 22 & $\begin{array}{l}3 \text { randomized, } \\
\text { controlled, } \mathrm{CO},\end{array}$ & Healthy women $(n=13-20)$ & OTCS & $\begin{array}{c}150 \mathrm{~g} \text { whole berries puree along each meal } \\
\text { study 1: white bread }+ \text { strawberries, } \\
\text { bilberries, or lingonberries } \\
\text { study 2: white bread }+\mathrm{h} \text { raspberries, } \\
\text { cloudberries, or chokeberries } \\
\text { study 3: white bread or rye bread }+ \text { mix } \\
\text { berries consisting of equal amounts of } \\
\text { strawberries, bilberries, cranberries, } \\
\text { and blackcurrants }\end{array}$ & $\begin{array}{l}\text { White bread or rye bread with } \\
50 \mathrm{~g} \text { available starch }\end{array}$ & $\begin{array}{l}\text { Reduced the postprandial insulin } \\
\text { response, improved the glycemic profile, } \\
\text { improved postprandial } \\
\text { glucose metabolism. }\end{array}$ & [93] \\
\hline
\end{tabular}


Table 1. Cont.

\begin{tabular}{|c|c|c|c|c|c|c|c|}
\hline No. & Study Design & Study Subject & Duration & Berry Interventions & Intervention Diet & Significant Findings & Ref. \\
\hline 23 & $\mathrm{CO}, \mathrm{RCT}$ & $\begin{array}{l}\text { T2DM men and women } \\
\qquad(n=\mathrm{NS})\end{array}$ & $12 \mathrm{wk}$ & $\begin{array}{c}250 \mathrm{~g} \text { frozen red raspberries puree with } \\
\text { each breakfast }\end{array}$ & NA & $\begin{array}{l}\text { Anti-dyslipidemic, anti-inflammatory, } \\
\text { anti-obesity }\end{array}$ & [94] \\
\hline \multicolumn{8}{|c|}{ (I) Mulberries (MBs) (Animal studies) } \\
\hline 1 & $\begin{array}{l}\text { Randomized } \\
\text { block design }\end{array}$ & Male C57BL/6 mice $(n=60)$ & $8 \mathrm{wk}$ & $\begin{array}{l}\text { MB anthocyanins at } 200 \mathrm{mg} / \mathrm{kg} \text { HFD } \\
\text { provided ad libitum }\end{array}$ & $\operatorname{HFD}(45 \%$ kcal from fat) & $\begin{array}{l}\text { Anti-dyslipidemia, anti-inflammatory, } \\
\text { anti-obesity }\end{array}$ & [95] \\
\hline 2 & $\mathrm{RCT}$ & $\begin{array}{c}\text { Male } \mathrm{db} / \mathrm{db} \text { mice with } \\
\text { C57BL6/J genetic background } \\
(n=50)\end{array}$ & $8 \mathrm{wk}$ & $\begin{array}{l}\text { MB fruit extracts } 50 \text { and } 125 \mathrm{mg} / \mathrm{kg} \text { BW } \\
\text { every day orally by gavage }\end{array}$ & NA & Antioxidative and hypoglycemic & [96] \\
\hline 3 & RCT & male adult Wistar rats $(n=70)$ & $6 \mathrm{wk}$ & $\begin{array}{l}\text { MB fruit wine } 400 \mathrm{~mL} / 70 \mathrm{~kg} \text { of body } \\
\text { weight daily }\end{array}$ & NA & Antioxidative and hypoglycemic & [97] \\
\hline 4 & RCT & $\begin{array}{l}\text { male Sprague-Dawley rats } \\
\qquad(n=50)\end{array}$ & $8 \mathrm{wk}$ & $\begin{array}{c}\text { MB fruit derived } \\
\text { cyanidin-3-O- } \beta \text {-D-glucopyranoside } \\
(10 \mathrm{mg} / \mathrm{kg} . \mathrm{BW} \text {. daily) orally by gavage }\end{array}$ & NA & Antidiabetic cystopathy & [98] \\
\hline 5 & RCT & $\begin{array}{l}\text { Adult diabetic male Wistar rats } \\
\qquad(n=12)\end{array}$ & $6 \mathrm{wk}$ & MB polysaccharides (200 mg/kg.BW daily) & HFD & $\begin{array}{c}\text { Improved oral glucose tolerance/insulin } \\
\text { resistance, bioactivities of superoxide } \\
\text { dismutase (SOD), catalase (CAT) and } \\
\text { glutathione peroxidase (GPx), } \\
\text { were increased }\end{array}$ & [99] \\
\hline 6 & RCT & $\begin{array}{c}\text { Male } \\
\text { Gold Syrian hamsters } \\
(n=\mathrm{NS})\end{array}$ & $12 \mathrm{wk}$ & $\begin{array}{l}\text { Water extracts of MB fruit at } 1-2 \%(w / w) \text { in } \\
\text { HFD provided ad libitum }\end{array}$ & $\begin{array}{l}\text { HFD }(1 \% \text { cholesterol and } 10 \% \\
\text { corn oil })\end{array}$ & Hypolipidemic & [100] \\
\hline 7 & RCT & Male C57BL/6 mice $(n=48)$ & $12 \mathrm{wk}$ & $\begin{array}{l}\text { Anthocyanin from MB of } 40-200 \mathrm{mg} / \mathrm{kg} \\
\text { of HFD }\end{array}$ & $\operatorname{HFD}(45 \%$ kcal from fat) & $\begin{array}{l}\text { Inhibit body weight gain, reduce the } \\
\text { resistance to insulin, lower the size of } \\
\text { adipocytes, attenuate lipid accumulation } \\
\text { and decrease the leptin secretion. }\end{array}$ & [101] \\
\hline 8 & $\mathrm{RCT}$ & $\begin{array}{l}\text { Male Syrian golden hamsters } \\
\qquad(n=32)\end{array}$ & $12 \mathrm{wk}$ & $\begin{array}{l}\text { Water extracts of MB fruit at } 0.5-2 \%(\mathrm{w} / \mathrm{w}) \\
\text { in HFD provided ad libitum }\end{array}$ & $\begin{array}{l}\text { HFD }(10 \% \text { corn oil }+0.1 \% \\
\text { cholesterol })\end{array}$ & Anti-obese and hypolipidemic effects & [102] \\
\hline 9 & RCT & Male Wistar rats $(n=32)$ & $4 \mathrm{wk}$ & $\begin{array}{l}5-10 \%(\mathrm{w} / \mathrm{w}) \text { mulberry fruit } \\
\text { polysaccharide fractions in HFD provided } \\
\text { ad libitum }\end{array}$ & $\begin{array}{l}\text { HFD }(10 \% \text { lard, } 1 \% \text { cholesterol, } \\
0.5 \% \text { sodium cholate, and } 88.5 \% \\
\text { commercial diet) }\end{array}$ & $\begin{array}{l}\text { Hypolipidemic and improved the } \\
\text { enzymatic antioxidant system }\end{array}$ & [103] \\
\hline 10 & RCT & Male C57BL/6 mice $(n=48)$ & $6 \mathrm{wk}$ & $\begin{array}{l}0.5-2 \%(\mathrm{w} / \mathrm{w}) \text { water extracts of MB fruit in } \\
\text { high-fat }(35 \% \mathrm{kcal} \text { from fat) ethanol rich } \\
\text { liquid diet ( } 36 \% \mathrm{kcal} \text { from ethanol) } \\
\text { provided ad libitum }\end{array}$ & $\begin{array}{l}\text { high-fat (35\% kcal from fat) } \\
\text { ethanol rich liquid diet } \\
\text { (36\%kcal from ethanol) }\end{array}$ & $\begin{array}{l}\text { Anti-obesity, hypoglycemic, } \\
\text { antioxidative, anti-inflammatory }\end{array}$ & [104] \\
\hline 11 & RCT & $\begin{array}{l}\text { Male Sprague-Dawley rats } \\
\qquad(n=40)\end{array}$ & $10 \mathrm{wk}$ & MB fruit extracts 100 or $200 \mathrm{mg} / \mathrm{kg}$. BW.day & $\begin{array}{l}\text { HFD ( } 1 \% \text { cholesterol, } 18 \% \text { lipid } \\
\text { (lard), } 40 \% \text { sucrose })\end{array}$ & $\begin{array}{c}\text { Anti-dyslipidemic, antioxidative, } \\
\text { ameliorates nonalcoholic fatty liver } \\
\text { disease (NAFLD) }\end{array}$ & [105] \\
\hline
\end{tabular}


Table 1. Cont.

\begin{tabular}{|c|c|c|c|c|c|c|c|}
\hline No. & Study Design & Study Subject & Duration & Berry Interventions & Intervention Diet & Significant Findings & Ref. \\
\hline 12 & $\mathrm{RCT}$ & Female Wistar rats $(n=48)$ & $20 \mathrm{wk}$ & $\begin{array}{l}\text { Microencapsulated } 50 \text { to } \\
250 \mathrm{mg} / \mathrm{kg} \text {.BW.day mulberry fruit extracts } \\
\text { (microencapsulated) with HCHF }\end{array}$ & $\begin{array}{c}\text { High-carbohydrate high-fat } \\
\text { (HCHF) diet which contained } \\
\text { total energy around } 4.62 \mathrm{kcal} / \mathrm{g} \\
\text { (fat } 31.54 \% \text {, protein } 20.25 \% \text {, and } \\
\text { carbohydrate } 48.21 \% \text { ). }\end{array}$ & $\begin{array}{l}\text { Anti-inflammatory, antioxidative, } \\
\text { improved metabolic syndrome }\end{array}$ & [106] \\
\hline 13 & RCT & Male, C57BL/6J mice $(n=12)$ & $13 \mathrm{wk}$ & $\begin{array}{l}20 \% \text { MB powder in HFD provided } \\
\text { ad libitum }\end{array}$ & HFD, $60 \%$ calories from fat & $\begin{array}{l}\text { Anti-obesity, antidiabetic, increase of } \\
\text { Bacteroidetes/Firmicutes ratio }\end{array}$ & [107] \\
\hline 14 & RCT & $\mathrm{db} / \mathrm{m}$ mice $(n=50)$ & NS & $\begin{array}{l}\text { MB fruit polysaccharide fractions } \\
\qquad(200-800 \mathrm{mg} / \mathrm{kg} . \mathrm{BW})\end{array}$ & NA & $\begin{array}{l}\text { Improved antioxidant enzymatic defense } \\
\text { system, antihyperglycemic and } \\
\text { antihyperlipidemic effects }\end{array}$ & [108] \\
\hline 15 & RCT & $\begin{array}{l}\text { Male C57BL6/J genetic } \\
\text { background }(\mathrm{db} / \mathrm{db}) \text { mice } \\
\qquad(n=60)\end{array}$ & $8 \mathrm{wk}$ & $\begin{array}{l}\text { Mulberry fruit extract } 25-250 \mathrm{mg} / \mathrm{kg} \\
\text { BW daily }\end{array}$ & NA & $\begin{array}{l}\text { Upregulation of gluconeogenesis } \\
\text { pathway }\end{array}$ & [109] \\
\hline 16 & RCT & $\begin{array}{l}\text { Adult diabetic male Wistar } \\
\qquad(n=40)\end{array}$ & $7 \mathrm{wk}$ & $\begin{array}{l}\text { MB fruit polysaccharide fractions MFP50 } \\
\text { and MFP90 (400 mg/kg.BW) }\end{array}$ & HFD & $\begin{array}{l}\text { Antihyperglycemic and } \\
\text { antihyperlipidemic effects }\end{array}$ & [110] \\
\hline \multicolumn{8}{|c|}{ (I) Lingonberries (LB) (Animal studies) } \\
\hline 1 & RCT & Male C57BL/6 mice $(n=\mathrm{NS})$ & $8 \mathrm{wk}$ & $\begin{array}{l}\text { LB extracts }(125,250 \text {, and } 500 \mathrm{mg} / \mathrm{kg}) \text { in } \\
\text { HFD provided ad libitum }\end{array}$ & $\begin{array}{c}\text { HFD ( } 35 \% \text { fat, } 20 \% \text { protein, and } \\
36.5 \% \text { carbohydrate) }\end{array}$ & $\begin{array}{l}\text { Attenuates hepatic steatosis } \\
\text { hyperglycemia, hyperlipidemia. } \\
\text { Improves insulin signaling }\end{array}$ & [111] \\
\hline 2 & $\mathrm{RCT}$ & SHR rats $(n=\mathrm{NS})$ & $8 \mathrm{wk}$ & $\begin{array}{l}\text { Cold-compressed LB juice provided } \\
\text { ad libitum }\end{array}$ & NA & $\begin{array}{l}\text { Reduced hypertension and } \\
\text { pro-inflammatory markers }\end{array}$ & [112] \\
\hline 3 & RCT & $\begin{array}{l}\text { Male C57BL/6JBomTac mice } \\
\qquad(n=120)\end{array}$ & $13 \mathrm{wk}$ & $\begin{array}{c}20 \%(w / w) \text { F/D LB in HFD provided } \\
\text { ad libitum }\end{array}$ & HFD ( $45 \mathrm{kcal} \%$ fat $)$ & $\begin{array}{l}\text { Significantly reduced body fat, lipid } \\
\text { accumulation, and plasma levels of the } \\
\text { inflammatory marker PAI-1, as well as } \\
\text { mediated positive effects on glucose } \\
\text { metabolism homeostasis. }\end{array}$ & [113] \\
\hline 4 & RCT & $\begin{array}{l}\text { Male C57BL/6JBomTac mice } \\
(n=\mathrm{NS})\end{array}$ & $11 \mathrm{wk}$ & $\begin{array}{c}20 \%(w / w) \text { F/D LB in HFD provided } \\
\text { ad libitum }\end{array}$ & HFD (45 kcal\% fat) & $\begin{array}{l}\text { Reduced plasma levels of markers of } \\
\text { endotoxemia and inflammation }\end{array}$ & [114] \\
\hline 5 & RCT & Male Apoe-/- mice $(n=35)$ & $8 \mathrm{wk}$ & $44 \%$ lingonberry + HFD & HFD ( $38 \mathrm{kcal} \%$ fat $)$ & $\begin{array}{l}\text { Decreased triglyceridemia and } \\
\text { reduced atherosclerosis }\end{array}$ & [115] \\
\hline 6 & $\mathrm{RCT}$ & $\begin{array}{l}\text { Male C57BL/6JBomTac mice } \\
(n=\mathrm{NS})\end{array}$ & $11 \mathrm{wk}$ & $\begin{array}{c}20 \%(w / w) \text { F/D LB in HFD provided } \\
\text { ad libitum }\end{array}$ & HFD ( $45 \mathrm{kcal} \%$ fat $)$ & $\begin{array}{l}\text { Improvement in glycaemia, reduction in } \\
\text { inflammation and hepatic steatosis }\end{array}$ & [116] \\
\hline 7 & RCT & C57BL/6JBomTac ( $n=\mathrm{NS})$ & 13 wk & $\begin{array}{c}20 \%(w / w) \text { freeze-dried LB + blackcurrants, } \\
\text { bilberries or açai berry in HFD provided } \\
\text { ad libitum }\end{array}$ & HFD (45 kcal\% fat) & $\begin{array}{l}\text { Downregulation of inflammatory } \\
\text { pathways, NF-kB, STAT3 and mTOR as } \\
\text { possible targets for antidiabetic therapy }\end{array}$ & [117] \\
\hline 8 & RCT & Male ApoE- $/-$ mice $(n=50)$ & $8 \mathrm{wk}$ & $\begin{array}{l}\text { Two LB polysaccharide fractions } \\
\text { 15-60 g/kg BW with HFD daily }\end{array}$ & $\operatorname{HFD}(38 \mathrm{kcal} \%$ fat $)$ & $\begin{array}{l}\text { Hypoglycemic, hypolipidemic, altered } \\
\text { caecal microbiota composition }\end{array}$ & [118] \\
\hline
\end{tabular}


Table 1. Cont.

\begin{tabular}{|c|c|c|c|c|c|c|c|}
\hline No. & Study Design & Study Subject & Duration & Berry Interventions & Intervention Diet & Significant Findings & Ref. \\
\hline \multicolumn{8}{|c|}{ Human studies } \\
\hline 9 & $\mathrm{RCT}$ & $\begin{array}{c}\text { Scandinavian type } 2 \text { diabetes } \\
\text { patients }(n=30)\end{array}$ & $12 \mathrm{wk}$ & $\begin{array}{l}\text { Recommended daily intake of } \\
\text { LB/berries/fruits }\end{array}$ & $\begin{array}{l}\text { Okinawan-based Nordic diet of } \\
\text { about } 1,900 \mathrm{kcal} / \text { day }\end{array}$ & $\begin{array}{l}\text { Improved metabolic and } \\
\text { anthropometric parameters }\end{array}$ & [119] \\
\hline 10 & $\mathrm{CO}, \mathrm{DB}, \mathrm{RCT}$ & $\begin{array}{l}\text { Healthy normal-weight } \\
\text { nonsmoking men }(n=\mathrm{NS})\end{array}$ & $6 \mathrm{~d}$ & $\begin{array}{l}\text { Glycemic diet }+40 \mathrm{~g} \text { lingonberry powder } \\
\text { Lipemic diet }+60 \mathrm{~g} \text { lingonberry powder }\end{array}$ & $\begin{array}{l}\text { Glycemic diet: } 200 \mathrm{~g} \text { yoghurt } \\
\text { (lactose-free and fat-free } \\
\text { non-flavored natural yoghurt + } \\
50 \mathrm{~g} \text { glucose) Lipemic diet: } \\
200 \mathrm{~g} \text { Yoghurt (lactose-free and } \\
\text { fat-free non-flavored natural } \\
\text { yoghurt + } 35 \text { canola oil) }\end{array}$ & $\begin{array}{l}\text { Nullified the glycemic effect of the sugars } \\
\text { present in the meals without affecting the } \\
\text { postprandial lipemic response }\end{array}$ & [120] \\
\hline 11 & $\mathrm{CO}, \mathrm{DB}, \mathrm{RCT}$ & $\begin{array}{l}13 \text { Healthy, over-weight, } \\
\text { non-smoking male and } \\
\text { female volunteers }\end{array}$ & $\begin{array}{l}\text { Single meal } \\
\text { challenge }\end{array}$ & $100 \mathrm{~g}$ lingonberry & $\begin{array}{c}\text { Hyperlipidic and } \\
\text { hypercaloric meals } \\
\text { (38 kcal\% fat) }\end{array}$ & $\begin{array}{l}\text { Reduced glycemic response, rarified the } \\
\text { increase of cholesterolemia }\end{array}$ & [121] \\
\hline 12 & $\mathrm{RCT}$ & $\begin{array}{l}\text { Normal, healthy subjects } \\
\qquad(n=9)\end{array}$ & $12 \mathrm{wk}$ & $\begin{array}{l}\text { LB polysaccharides }+ \text { fibers } \\
(2 \mathrm{~g} / \mathrm{Kg} \text { of oat bread })\end{array}$ & Oat bread & $\begin{array}{l}\text { In reduced glucose and } \\
\text { C-peptide response }\end{array}$ & [122] \\
\hline 13 & $\mathrm{SB}, \mathrm{CO}, \mathrm{RCT}$ & $\begin{array}{l}\text { Healthy women volunteers } \\
\qquad(n=20)\end{array}$ & $\begin{array}{l}\text { 2-h meal } \\
\text { tests }\end{array}$ & $\begin{array}{l}\text { Diet 1: } 150 \mathrm{~g} \text { whole LB puree containing } \\
\quad 35 \mathrm{~g} \text { sucrose per day } \\
\text { Diet 2: } 300 \mathrm{~mL} \text { LB nectar (equal to } 150 \mathrm{~g} \\
\text { fresh berries) containing } 35 \mathrm{~g} \text { sucrose }\end{array}$ & NA & $\begin{array}{l}\text { Optimized postprandial metabolic } \\
\text { responses to sucrose with delayed } \\
\text { digestion and absorption of } \\
\text { sucrose/glucose }\end{array}$ & [123] \\
\hline 14 & RCT & $\begin{array}{l}\text { Healthy non-smoking males } \\
\qquad(n=14)\end{array}$ & $\begin{array}{l}\text { 2-h meal } \\
\text { tests }\end{array}$ & $\begin{array}{l}60 \mathrm{~g} \text { of } \mathrm{LB} \text { juice press residue } \\
\text { corresponding to } 270 \mathrm{~g} \text { of fresh LB with } \\
\text { standard diet }\end{array}$ & $\begin{array}{l}\text { Standard diet: white wheat } \\
\text { bread, cucumber, water, } \\
\text { and a banana }\end{array}$ & $\begin{array}{c}\text { Gut microfloral metabolism of } \\
\text { polyphenols resulting in increased levels } \\
\text { of hippuric acid and } \\
\text { 4-hydroxyhippuric acid }\end{array}$ & [124] \\
\hline \multicolumn{8}{|c|}{ (I) Blackberries (BBR) (Animal studies) } \\
\hline 1 & $\mathrm{RCT}$ & Male Wistar rats $(n=32)$ & $5 \mathrm{wk}$ & $\begin{array}{l}\text { Anthocyanin-enriched fraction (AF) and } \\
\text { Ellagitannin-enriched fraction (EF) } \\
\text { equivalent to ( } 4 \mathrm{mg} \text { cyanidin eq/kg BW) } \\
\text { and } 2.68 \mathrm{mg} \text { EA eq } / \mathrm{kg} \text { BW respectively }\end{array}$ & NA & $\begin{array}{l}\text { Reinforce the antioxidative defense } \\
\text { system and lipid oxidation markers }\end{array}$ & [125] \\
\hline 2 & RCT & C57BL/6 mice $(n=60)$ & $12 \mathrm{wk}$ & $\begin{array}{l}\text { BBR extracts at } 200 \mathrm{mg} / \mathrm{kg} \text { food BBR } \\
\text { extracts: cyanidin-3-glucoside }(51.24 \%), \\
\text { cyanidin-3-rutinoside }(42.31 \%), \text { and } \\
\text { peonidin-3glucoside }(6.91 \%)\end{array}$ & $\operatorname{HFD}(45 \%$ kcal from fat) & $\begin{array}{l}\text { Anti-inflammatory, anti-hypertensive, } \\
\text { anti-hypercholesterolemia, antioxidative }\end{array}$ & [126] \\
\hline 3 & RCT & $\begin{array}{l}\text { Male DIO C57BL/6J mice } \\
\qquad(n=40)\end{array}$ & $12 \mathrm{wk}$ & $\begin{array}{l}\text { 6.3\%, (w/w) BBR extracts in HFD provided } \\
\text { ad libitum }\end{array}$ & $\operatorname{HFD}(45 \%$ kcal from fat) & $\begin{array}{l}\text { Anti-obesity, Anti-inflammatory, } \\
\text { anti-hypertensive, }\end{array}$ & [127] \\
\hline 4 & RCT & Male Wistar rats $(n=24)$ & $17 \mathrm{wk}$ & $\begin{array}{l}25 \mathrm{mg} / \mathrm{kg} . \text { BW BBR extracts in HFD } \\
\text { provided ad libitum }\end{array}$ & $\operatorname{HFD}(45 \%$ kcal from fat $)$ & $\begin{array}{l}\text { Anti-obesity, anti-inflammatory, } \\
\text { anti-dyslipidemic }\end{array}$ & [128] \\
\hline
\end{tabular}


Table 1. Cont.

\begin{tabular}{|c|c|c|c|c|c|c|c|}
\hline No. & Study Design & Study Subject & Duration & Berry Interventions & Intervention Diet & Significant Findings & Ref. \\
\hline 5 & RCT & $\begin{array}{l}\text { Male diabetic Sprague-Dawley } \\
\text { rats }(n=40)\end{array}$ & $40 \mathrm{~d}$ & Microfiltrated 12.5-25\% BBR juices & & $\begin{array}{c}\text { Reduced glycaemia }(-10.4 \%), \text { TG }(-4.6 \%) \\
\text { and TC }(21.0 \%), \text { lipid peroxidation, } \\
\text { attenuation of oxidative stress }\end{array}$ & [129] \\
\hline 6 & RCT & Male Wistar strain rats $(n=40)$ & $4 \mathrm{wk}$ & $\begin{array}{l}\text { Normal standard diet with } 0.98 \% \text { BBR } \\
\text { polyphenols and } 6 \% \text { BBR fiber }\end{array}$ & Normal chow diet & $\begin{array}{l}\text { Anti-inflammatory and } \\
\text { anti-dyslipidemic }\end{array}$ & [130] \\
\hline 7 & $\mathrm{RCT}$ & $\begin{array}{c}\text { Female obese } \\
\text { (BKS(D)-Leprdb/J72) and lean } \\
\text { (C57BL/6J) mice }(n=24)\end{array}$ & $10 \mathrm{wk}$ & $\begin{array}{l}\text { Aged or fresh BBR supplemented at } 10 \% \\
\text { (w/w) of diet provided ad libitum }\end{array}$ & Normal chow diet & $\begin{array}{c}\text { Increased in total beneficial bacterial } \\
\text { population }\end{array}$ & [131] \\
\hline 8 & RCT & Male C57BL/6J mice $(n=72)$ & $10 \mathrm{wk}$ & $\begin{array}{c}\text { Alcohol-free blueberry-blackberry } \\
\text { fermented beverage (AFFB) a) AFFB } \\
\text { [70\% blackberry and 30\% blueberry, } 8.4 \mathrm{mg} \\
\text { cyanidin-3-O-glucoside (C3G) } \\
\text { eq./kg.BW)/day]; (b) dose } 0.1 \times \\
\text { ostamberlite extract (PAE), } 1.1 \mathrm{mgC} 3 \mathrm{G} \\
\text { eq./kg BW/day; (c) dose } 1 \times \text { PAE, } 9.0 \mathrm{mg} \\
\text { C3G eq./kg BW/day; (d) dose } 2 \times \text { PAE, } \\
18.9 \mathrm{mg} \text { C3G eq./kg BW/day }\end{array}$ & $\begin{array}{l}\text { HFD }(60.3 \% \text { fat, } 21.3 \% \\
\text { carbohydrate and } 18.4 \% \\
\text { protein) }\end{array}$ & $\begin{array}{l}\text { Reduced percent fat mass, mean } \\
\text { adipocyte diameters, epididymal fat pad } \\
\text { weights, and plasma TG and TC. }\end{array}$ & [22] \\
\hline \multicolumn{8}{|c|}{ Human studies } \\
\hline 9 & RCT & $\begin{array}{l}\text { Diabetic and obese men and } \\
\quad \text { women }(n=152)\end{array}$ & $1 \mathrm{wk}$ & $\begin{array}{l}\text { Consumption of daily recommended } \\
\text { amount of low glycemic index fruit } \\
(0.7-1.4 \text { servings/day })\end{array}$ & NA & Anti-dyslipidemic & [132] \\
\hline 10 & $\begin{array}{c}\text { open, } \\
\text { single-center } \\
\text { RCT }\end{array}$ & Healthy human subjects $(n=6)$ & $4 \mathrm{~h}$ & $\begin{array}{l}200 \mathrm{~mL} \text { of BBR juice equivalent to } 400 \mathrm{mg} \\
\text { of cyanidin equivalent } 50 \mathrm{~kg} \text { of } \\
\text { body weight }\end{array}$ & NA & $\begin{array}{l}\text { Improved plasma and urine antioxidant } \\
\text { system }\end{array}$ & [133] \\
\hline 11 & RCT & Dyslipidemic patients $(n=72)$ & $8 \mathrm{wk}$ & $\begin{array}{l}300 \mathrm{~mL} \text { of BBR juice (equivalent to } \\
316 \mathrm{mg} / 100 \text { g polyphenols) of BBR with } \\
\text { pulp every day }\end{array}$ & NA & $\begin{array}{l}\text { Increased apo A-1 and HDL-C along } \\
\text { reduction in apo B and hsCRP }\end{array}$ & [134] \\
\hline \multicolumn{8}{|c|}{ (I) Strawberries (SB) (Animal studies) } \\
\hline 1 & RCT & $\begin{array}{l}\text { Diabetic male albino Wistar } \\
\text { rats }(n=36)\end{array}$ & $4 \mathrm{wk}$ & $\begin{array}{l}\text { Aqueous, alcoholic and hydro-alcoholic SB } \\
\text { extract }(2 \mathrm{~g} / \mathrm{kg} \text { b.w.day }\end{array}$ & NA & $\begin{array}{l}\text { Reduced expression level of genes } \\
\text { involving glucose, lipid metabolism with } \\
\text { improvement in glucose metabolism and } \\
\text { liver function }\end{array}$ & [135] \\
\hline 2 & RCT & Male Wistar rats $(n=20)$ & $12 \mathrm{wk}$ & $\begin{array}{l}\text { HFD supplemented with } 0.2 \% \\
\text { irradiated/non-irradiated SB extracts }\end{array}$ & $\operatorname{HFD}(47.5 \%$ kcal from fat $)$ & $\begin{array}{l}\text { Reduction in the oxidative damage in } \\
\text { brain and peripheral tissues }\end{array}$ & [136] \\
\hline 3 & RCT & Male C57BL/6J mice $(n=36)$ & $24 \mathrm{wk}$ & $\begin{array}{l}\text { HFD supplemented with } 2.6 \% \\
\text { freeze-dried SB }\end{array}$ & $\begin{array}{l}\text { HFD containing approximately } \\
20 \% \text { higher in energy density } \\
\text { compared to the low-fat diets }\end{array}$ & $\begin{array}{l}\text { Reduction in the HFD led increase of } \\
\text { FBS, adhesion molecule-1, leptin, } \\
\text { E-selectin, resistin, and plasminogen } \\
\text { activator protein-1 }\end{array}$ & [137] \\
\hline
\end{tabular}


Table 1. Cont.

\begin{tabular}{|c|c|c|c|c|c|c|c|}
\hline No. & Study Design & Study Subject & Duration & Berry Interventions & Intervention Diet & Significant Findings & Ref. \\
\hline 4 & $\mathrm{RCT}$ & Male Wistar rats $(n=48)$ & $8 \mathrm{wk}$ & $\begin{array}{l}\text { Supplementation of the diet with a } 6 \% \mathrm{w} / \mathrm{w} \\
\text { (equivalent to a } 5 \mathrm{~g} / \mathrm{kg} 65 \mathrm{BW} \text { dose) of a } \\
\text { F/D SB-BlB (5:1) powder (FDSB) }\end{array}$ & $\begin{array}{l}\text { High-fat-sucrose diet (D12451, } \\
\text { Research Diet) }\end{array}$ & $\begin{array}{l}\text { Anti-obesogenic and } \\
\text { anti-inflammatory effects }\end{array}$ & [138] \\
\hline 5 & $\mathrm{RCT}$ & Male Wistar rats $(n=24)$ & $16 \mathrm{wk}$ & $\begin{array}{l}\text { AIN93-modified diet with lyophilized SB } \\
\text { extract at } 10 \mathrm{~g} / \mathrm{kg} \text { of diet }\end{array}$ & AIN93-modified diet & $\begin{array}{l}\text { Improvement of oxidative stress } \\
\text { biomarkers, mitochondrial performance, } \\
\text { antioxidant enzyme activities, reduction } \\
\text { of DNA damage and ROS concentration }\end{array}$ & [139] \\
\hline 6 & $\mathrm{RCT}$ & Male Wistar rats $(n=20)$ & $12 \mathrm{wk}$ & Supplementation of $0.2 \%$ SB & $\begin{array}{l}\text { HFD ( } 47.5 \% \text { calories } \\
\text { from fat) }\end{array}$ & Antioxidative, anti-stress & [140] \\
\hline 7 & RCT & German Landrace pigs $(n=48)$ & $4 \mathrm{wk}$ & $205-745 \mathrm{~g}$ of SB with normal feed per day & $\begin{array}{l}\text { Linseed oil ( } 15 \mathrm{~g} / \text { day) enriched } \\
\text { feed }\end{array}$ & Anti-stress and antioxidative & [22] \\
\hline 8 & RCT & $\begin{array}{l}\mathrm{db} / \mathrm{db} \text { mice homozygous for } \\
\text { the diabetes spontaneous } \\
\text { mutation (Leprdb) with } \\
\text { C57BL/6J background }(n=24)\end{array}$ & $10 \mathrm{wk}$ & $\begin{array}{l}\text { 2.35\% F/D SB powder in the diet pellets } \\
(w / w) \text { (equivalent to two human servings of } \\
\text { SB i.e., } \sim 160 \mathrm{~g} \mathrm{SB})\end{array}$ & NA & Increased Bacteriodetes to Firmicutes ratio & [141] \\
\hline 9 & RCT & Male CD-1 mice $(n=60)$ & $8 \mathrm{wk}$ & $\begin{array}{c}5 \%(w / w) \text { of diet freeze-dried whole } \\
\text { SB powder }\end{array}$ & AIN93G diet & Increased Bacteriodetes to Firmicutes ratio & [142] \\
\hline \multicolumn{8}{|c|}{ (Human studies) } \\
\hline 10 & $\begin{array}{l}\text { DB, RCT, } \\
\text { parallel study }\end{array}$ & $\begin{array}{l}\text { Insulin resistant and obese } \\
\text { males and females }(n=41)\end{array}$ & $6 \mathrm{wk}$ & $\begin{array}{l}\text { Beverage containing } 1.84 \mathrm{~g} \text { of a mixture of } \\
\text { dry SB and CrB providing } 333 \mathrm{mg} \text { of } \\
\text { polyphenols on daily basis (also equivalent } \\
\text { to } 112 \mathrm{~g} \text { consumption of fresh berry fruit) }\end{array}$ & NA & Improved insulin sensitivity and release & [143] \\
\hline 11 & $\mathrm{CO}, \mathrm{SB}, \mathrm{PC}, \mathrm{RCT}$ & $\begin{array}{l}\text { Hyperlipidemic men and } \\
\text { women }(n=24)\end{array}$ & $12 \mathrm{wk}$ & $\begin{array}{l}\text { SB beverage containing } 10 \mathrm{~g} / \text { serving of } \\
\text { freeze-dry SB powder providing } 338 \mathrm{mg} \text { of } \\
\text { polyphenols daily (also equivalent to } 110 \mathrm{~g} \\
\text { consumption of fresh berry fruit) }\end{array}$ & $\begin{array}{l}\text { HFD consisting of typical } \\
\text { breakfast food items (i.e., bagel, } \\
\text { cream cheese, whole milk, egg, } \\
\text { margarine, cantaloupe) }\end{array}$ & $\begin{array}{l}\text { Reduced postprandial lipemia and } \\
\text { oxidative stress markers }\end{array}$ & [144] \\
\hline 12 & $\mathrm{CO}, \mathrm{RCT}$ & $\begin{array}{l}\text { Healthy males and females } \\
\qquad(n=30)\end{array}$ & $5 \mathrm{~d}$ & $\begin{array}{l}20 \mathrm{~g} \text { of five types SB jams each with sugar } \\
\text { of different glycemic index }\end{array}$ & $60 \mathrm{~g}$ white bread slice & $\begin{array}{l}\text { Non-significant reduction in the } \\
\text { postprandial glucose level }\end{array}$ & [145] \\
\hline 13 & CO DB RCT & $\begin{array}{l}\text { Healthy males and females } \\
\qquad(n=16)\end{array}$ & $3 \mathrm{wk}$ & $\begin{array}{c}60 \mathrm{~g} \text { of three types SB jams each with sugar } \\
\text { of different glycemic index and } \\
\text { polyphenolic contents }\end{array}$ & & $\begin{array}{l}\text { Strawberry jam with high sugar level } \\
\text { produced less levels of FFA. }\end{array}$ & [146] \\
\hline 14 & DB RCT & $\begin{array}{l}\text { T2DM males and female } \\
\text { subjects }(n=36)\end{array}$ & $6 \mathrm{wk}$ & $\begin{array}{l}\text { Two cups of F/D SB beverage containing } \\
\qquad 25 \mathrm{~g} \times 2=50 \mathrm{~g}\end{array}$ & NA & $\begin{array}{l}\text { Reduction in LDL-C and LDL-C/TC and } \\
\text { LDL-C/HDL-C ratio }\end{array}$ & [147] \\
\hline 15 & $\begin{array}{l}\text { SB, CO parallel, } \\
\text { RCT }\end{array}$ & $\begin{array}{l}\text { Obese and overweight men } \\
\text { and women }(n=24)\end{array}$ & $6 \mathrm{wk}$ & $\begin{array}{l}\text { SB beverage containing } 10 \mathrm{~g} / \mathrm{serving} \text { of } \\
\text { freeze-dry SB powder providing } 96 \mathrm{mg} \text { of } \\
\text { polyphenols on daily (also equivalent to } \\
100 \mathrm{~g} \text { consumption of fresh berry fruit) }\end{array}$ & High-carbohydrate-fat diet & $\begin{array}{l}\text { Attenuation of diet-induced } \\
\text { inflammatory markers }\end{array}$ & [148] \\
\hline
\end{tabular}


Table 1. Cont

\begin{tabular}{|c|c|c|c|c|c|c|c|}
\hline No. & Study Design & Study Subject & Duration & Berry Interventions & Intervention Diet & Significant Findings & Ref. \\
\hline 16 & $\begin{array}{l}\text { Single-center, } \\
\mathrm{CO}, \mathrm{SB}, \mathrm{PC} \text {, }\end{array}$ & Men and women $(n=26)$ & OTCS & $\begin{array}{l}\text { SB Milk based beverage containing } \\
10 \mathrm{~g} / 305 \mathrm{~mL} \text { of F/D SB powder }\end{array}$ & $\begin{array}{c}\text { high-carbohydrate, } \\
\text { moderate-fat meal (HCFM) }\end{array}$ & $\begin{array}{l}\text { Reduced postprandial insulin and } \\
\text { inflammatory response }\end{array}$ & [149] \\
\hline 17 & $\begin{array}{l}\text { Four-arm, SB, } \\
\mathrm{PC}, \mathrm{CO}, \mathrm{RCT}\end{array}$ & $\begin{array}{l}\text { Males and females with } \\
\text { insulin resistance }(n=23)\end{array}$ & NS & $\begin{array}{l}\text { SB milkshake containing } 10-40 \mathrm{~g} \\
\text { freeze-dried } \mathrm{SB} \text { powder where } 10 \mathrm{~g} \text { freeze } \\
\text { dried powder }=110 \mathrm{~g} \text { fresh strawberries }\end{array}$ & Standard western type meal & $\begin{array}{l}\text { Reduced lipid oxidation and post-meal } \\
\text { insulin demand }\end{array}$ & [150] \\
\hline 18 & $\begin{array}{l}\text { Observatory } \\
\text { study }\end{array}$ & $\begin{array}{l}\text { Healthy men and women } \\
\qquad(n=247)\end{array}$ & 20 years & $\begin{array}{l}\text { Dietary flavonoids intake ( } 47-560 \mathrm{mg} / \text { day) } \\
\text { from fruits and berries }\end{array}$ & - & $\begin{array}{l}\text { Flavonoid Compounds in Driving } \\
\text { Patterns of Microbial } \\
\text { Community Assembly }\end{array}$ & [151] \\
\hline 19 & $\mathrm{RCT}$ & $\begin{array}{l}\text { Obese men and women } \\
\qquad(n=66)\end{array}$ & $12 \mathrm{wk}$ & $\begin{array}{l}\text { SB beverage containing } 25-50 \mathrm{~g} \text { freeze-dry } \\
\text { SB powder daily }\end{array}$ & $\operatorname{HFD}(50 \%$ calories from fat $)$ & $\begin{array}{l}\text { Increased the glutathione level, serum } \\
\text { catalase activity, and plasma } \\
\text { antioxidant capacity }\end{array}$ & [152] \\
\hline 20 & DB RCT & T2DM patients $(n=40)$ & $6 \mathrm{wk}$ & $\begin{array}{l}50 \mathrm{~g} \text { of freeze-dried SB powder (equivalent } \\
\text { to } 500 \mathrm{~g} \text { fresh strawberries) each day }\end{array}$ & NA & $\begin{array}{l}\text { Reduction in the markers of lipid } \\
\text { peroxidation (MDA), inflammatory } \\
\text { markers (CRP). Reducing trend } \\
\text { in HbA1c. }\end{array}$ & [153] \\
\hline \multicolumn{8}{|c|}{ (I) Goji berries (GB) (Animal studies) } \\
\hline 1 & RCT & $\begin{array}{c}\text { Alloxan-induced } \\
\text { hyperglycemic/hyperlipidemic } \\
\text { adult rabbits }(n=35) \text { and male } \\
\text { mice }(n=24)\end{array}$ & $10 \mathrm{~d}$ & $\begin{array}{l}\text { Water decoction }(0.25 \mathrm{~g} / \mathrm{kg} \text { BW day), crude } \\
\text { GB polysaccharides (10 mg/kg BW day), } \\
\text { and purified GB polysaccharides } \\
(10 \mathrm{mg} / \mathrm{kg} \text { BW day })\end{array}$ & NA & $\begin{array}{l}\text { Hypoglycemic and hypolipidemic effect } \\
\text { with increased plasma } \\
\text { antioxidant capacity }\end{array}$ & [154] \\
\hline 2 & RCT & Male Wistar rats $(n=70)$ & $8 \mathrm{wk}$ & $\begin{array}{l}\text { Ethanolic and aqueous GB extracts at } \\
50 \mathrm{mg} / \mathrm{kg} \text { b.w. or } 100 \mathrm{mg} / \mathrm{kg} \text { BW daily }\end{array}$ & HFD & $\begin{array}{l}\text { Significantly reduced liver damage and } \\
\text { oxidative changes }\end{array}$ & [155] \\
\hline 3 & $\mathrm{RCT}$ & $\begin{array}{l}\text { Diabetic male mice of original } \\
\text { Kun-ming strain }(n=\mathrm{NS})\end{array}$ & $4 \mathrm{wk}$ & $\begin{array}{c}\text { GB polysaccharides } \\
\text { (20-40 mg/kg BW day) orally }\end{array}$ & NA & Hypoglycemic and hypolipidemic & [156] \\
\hline 4 & $\begin{array}{l}\text { randomized } \\
\text { block design }\end{array}$ & $\begin{array}{l}\text { Obese male Sprague-Dawley } \\
\text { rats }(n=60)\end{array}$ & $8 \mathrm{wk}$ & GB anthocyanins at $50-200 \mathrm{mg} / \mathrm{kg}$ BW.day & HFD & $\begin{array}{l}\text { Reduced body-weight-gain with } \\
\text { anti-inflammatory properties }\end{array}$ & [157] \\
\hline 5 & RCT & $\begin{array}{l}\text { STZ-diabetic Male Wistar rats } \\
\qquad(n=\mathrm{NS})\end{array}$ & $8 \mathrm{wk}$ & GB polysaccharides $(10 \mathrm{mg} / \mathrm{kg}$, BW.day) & NA & $\begin{array}{l}\text { Increased antioxidative scavenging and } \\
\text { antioxidant enzymes. Increased activity } \\
\text { of protein kinase C (PKC) }\end{array}$ & [158] \\
\hline 6 & RCT & $\begin{array}{l}\text { STZ-induced diabetic } \\
\text { Sprague-Dawley male rats } \\
\quad(n=60)\end{array}$ & $8 \mathrm{wk}$ & Water decoction of GB ( $5 \mathrm{~g} / \mathrm{kg} . \mathrm{BW}$.day) & NA & Protective effects in diabetic retinopathy & [159] \\
\hline 8 & $\mathrm{RCT}$ & Male Wistar rats $(n=16)$ & $4 \mathrm{wk}$ & $\begin{array}{l}\text { GB polysaccharides } 10 \mathrm{mg} / \mathrm{kg} \text { BW.day } \\
\text { dissolved in physiological saline }\end{array}$ & High-fat-sucrose diet & $\begin{array}{l}\text { hypoglycemic and improving } \\
\text { hyperinsulinemia }\end{array}$ & [160] \\
\hline 9 & RCT & $\begin{array}{l}\text { Diabetic male C57BL/6J mice } \\
\qquad(n=48)\end{array}$ & $7 \mathrm{wk}$ & $\begin{array}{l}\text { GB polysaccharides } 100-500 \mathrm{mg} / \mathrm{kg} \\
\text { BW.day by gastric perfusion }\end{array}$ & HFD & $\begin{array}{l}\text { Hypoglycemic effects with increased } \\
\text { insulin-sensitizing, glucose metabolism, } \\
\text { insulin secretion, and promoting } \\
\text { pancreatic cell proliferation. }\end{array}$ & [26] \\
\hline
\end{tabular}


Table 1. Cont

\begin{tabular}{|c|c|c|c|c|c|c|c|}
\hline No. & Study Design & Study Subject & Duration & Berry Interventions & Intervention Diet & Significant Findings & Ref. \\
\hline 10 & RCT & Swiss Albino rat $(n=30)$ & $3 \mathrm{wk}$ & $\begin{array}{c}\text { Water-soluble polysaccharides } \\
\text { (galactomannan) } 250-500 \mathrm{mg} / \mathrm{kg} \text { BW.day } \\
\text { by oral gavage }\end{array}$ & NA & $\begin{array}{l}\text { Hypolipidemic, reduced lipid oxidation, } \\
\text { increased insulin-sensitizing and serum } \\
\text { antioxidant level }\end{array}$ & [161] \\
\hline 11 & RCT & Diabetic Wistar rats $(n=48)$ & $8 \mathrm{wk}$ & $\begin{array}{l}\text { Water-soluble GB polysaccharides } \\
250-500 \mathrm{mg} / \mathrm{kg} \text { BW.day by oral gavage }\end{array}$ & $\begin{array}{l}\text { HFD and HCD }(12 \% \text { protein, } \\
5 \% \text { fat, } 67 \% \text { carbohydrate, } \\
5 \% \text { cholesterol, } \\
\text { and } 5 \% \text { other additives })\end{array}$ & $\begin{array}{l}\text { Reduced serum level of IL-2, IL-6, } \\
\text { TNF- } \alpha \text {, IFN- } \alpha, \text { MCP- } 1 \text {, and ICAM- } 1 \text { with } \\
\text { increased activities of SOD and } \\
\text { GSH-Px activities }\end{array}$ & [162] \\
\hline 12 & $\mathrm{RCT}$ & $\begin{array}{l}\text { Postnatal Royal College of } \\
\text { Surgeons (RCS) rats }(n=60)\end{array}$ & $4 \mathrm{wk}$ & Whole GB powder $1 \mathrm{mg} / \mathrm{kg}$ of per day & NA & $\begin{array}{l}\text { Reduced Caspase- } 2 \text { activity in } \\
\text { experimental group at 25th } \\
\text { post-neonatal day }\end{array}$ & [163] \\
\hline 13 & RCT & $\begin{array}{l}\text { Male IL-10-deficient mice } \\
\qquad(n=14)\end{array}$ & $10 \mathrm{wk}$ & Diet supplemented with $1 \%$ GB & Normal diet & $\begin{array}{l}\text { Increased gut population of SCFA } \\
\text { producing bacteria }\end{array}$ & [164] \\
\hline \multicolumn{8}{|c|}{ Human studies } \\
\hline 14 & $\mathrm{RCT}$ & $\begin{array}{l}\text { Kunming mice of clean grade } \\
\qquad(n=14)\end{array}$ & $2 \mathrm{wk}$ & $\begin{array}{l}\text { GBPS at a dose of } 0.1 \mathrm{~mL} / 10 \mathrm{~g} \text { body weigh } \\
\text { daily via intragastric administration }\end{array}$ & Normal diet & $\begin{array}{l}\text { Increased gut population of SCFA } \\
\text { producing bacteria, Firmicutes, } \\
\text { Akkermansia, Lactobacillus, } \\
\text { and Prevotellaceae }\end{array}$ & [165] \\
\hline 15 & $\mathrm{DB}, \mathrm{PC}, \mathrm{RCT}$ & $\begin{array}{l}\text { Healthy males and females } \\
\qquad(n=50)\end{array}$ & $30 \mathrm{~d}$ & $\begin{array}{c}\text { Intake of } 120 \mathrm{~mL} \text { of GB juice (equivalent to } \\
1632 \mathrm{mg} / \text { daily serving }(120 \mathrm{~mL}) \text { of goji } \\
\text { berry polyphenols }\end{array}$ & $\begin{array}{l}\text { Traditional Chinese diet rich } \\
\quad \text { in carbohydrate }\end{array}$ & $\begin{array}{l}\text { Increased serum levels of glutathione } \\
\text { peroxidase (GSH-Px) and superoxide } \\
\text { dismutase (SOD) with reduced level } \\
\text { of MDA }\end{array}$ & [166] \\
\hline 16 & $\mathrm{RCT}$ & $\begin{array}{l}\text { Metabolic syndrome patients } \\
\qquad(n=\mathrm{NS})\end{array}$ & $45 \mathrm{~d}$ & $14 \mathrm{~g}$ of GB with meals & Normal diet & $\begin{array}{l}\text { Reduction in transaminases, waist } \\
\text { circumference with improvements in } \\
\text { lipid profile, glutathione and } \\
\text { catalase level. }\end{array}$ & [167] \\
\hline 17 & $\mathrm{RCT}$ & $\begin{array}{l}\text { Male and female C57BL/b6N } \\
\text { mice }(n=56)\end{array}$ & $8 \mathrm{wk}$ & $\begin{array}{c}\text { GB polysaccharides } \\
\text { (1-10 } \mathrm{mg} / \mathrm{kg} \text { BW day) orally }\end{array}$ & NA & $\begin{array}{l}\text { Increased hepatic antioxidant enzymes, } \\
\text { y inhibited cytochrome P450 2E1, nitric } \\
\text { oxide metabolism and lipid peroxidation }\end{array}$ & [168] \\
\hline 18 & $\mathrm{DB}, \mathrm{CO}, \mathrm{RCT}$ & $\begin{array}{l}\text { healthy overweight men } \\
\qquad(n=\mathrm{NS})\end{array}$ & $\begin{array}{l}\text { Single meal } \\
\text { challenge }\end{array}$ & meal containing $25 \mathrm{~g}$ of dried GB fruit & $\begin{array}{l}\text { Ready-made meal with a fixed } \\
\text { macronutrient composition } \\
(30-40 \% \text { fat, } 40-50 \% \\
\text { carbohydrates, and } \\
13-16 \% \text { proteins })\end{array}$ & $\begin{array}{l}\text { No-single-dose-effect on substrate } \\
\text { oxidation and } \\
\text { prospandial-energy-expenditure }\end{array}$ & [169] \\
\hline
\end{tabular}


Table 1. Cont

\begin{tabular}{|c|c|c|c|c|c|c|c|}
\hline No. & Study Design & Study Subject & Duration & Berry Interventions & Intervention Diet & Significant Findings & Ref. \\
\hline \multicolumn{8}{|c|}{ (I) Acai berries (AB) (Animal studies) } \\
\hline 1 & $\mathrm{RCT}$ & $\begin{array}{l}\text { Male mice of the } \\
\text { C57BL/6 strain } \\
(n=\mathrm{NS})\end{array}$ & $12 \mathrm{wk}$ & $\begin{array}{l}\text { AB seed extracts } 300 \mathrm{mg} / \mathrm{kg} . \mathrm{BW} \text {.day by } \\
\text { intragastric gavage }\end{array}$ & $\operatorname{HFD}(60 \%$ calorie from fat $)$ & $\begin{array}{c}\text { Reduced expressions of lipogenic } \\
\text { proteins (SREBP-1c, pACC, ACC, } \\
\text { HMG-CoA reductase) with increased } \\
\text { expression of pAMPK, pACC/ACC, and } \\
\text { cholesterol transporters } \\
\text { (ABCG5 and ABCG8) }\end{array}$ & [170] \\
\hline 2 & $\mathrm{RCT}$ & Zebrafish $(n=70)$ & $5 \mathrm{wk}$ & $\begin{array}{l}\text { HC diet supplemented with } 10 \% \mathrm{w} / \mathrm{w} \text { of } \\
\text { AB puree powder }\end{array}$ & $\begin{array}{l}\text { high cholesterol (HC) diet } \\
(47.5 \% \text { crude protein, } 6.5 \% \\
\text { crude fat, } 4 \% \text { cholesterol, } 2.0 \% \\
\text { crude fiber, } 10.5 \% \text { crude ash) }\end{array}$ & $\begin{array}{c}\text { Reduced oxidative markers with lipid } \\
\text { lowering effects }\end{array}$ & [171] \\
\hline 3 & $\mathrm{RCT}$ & $\begin{array}{l}\text { Oxidatively damaged } \\
\text { sod1/sod1 mutant strains } \\
\text { Drosophila melanogaster } \\
\quad(n=120)\end{array}$ & $5-6 \mathrm{~d}$ & $\begin{array}{l}\text { AB supplemented sugar-yeast }(\mathrm{SY}) \\
\text { medium to a final concentration of } 0.25 \% \text {, } \\
0.5 \%, 1 \% \text { or } 2 \%(\mathrm{w} / \mathrm{v}) \text { of the food }\end{array}$ & SY medium & $\begin{array}{c}\text { Increased transcript level of } \\
\text { gluconeogenesis gene } \\
\text { phosphoenolpyruvate carboxykinase } \\
\text { (Pepck) with reduction in oxidative stress }\end{array}$ & [172] \\
\hline 4 & $\mathrm{RCT}$ & $\begin{array}{l}\text { ApoE-deficient }(\text { ApoE } 2 / 2) \\
\text { male mice }(n=23)\end{array}$ & $12 \mathrm{wk}$ & $\begin{array}{c}\text { AIN-93M diet formulated to contain } 2 \% \\
\text { F/D açai' pulp + exercise in progressive } \\
\text { treadmill for } 30 \text { min daily at a speed of } 12 \\
\mathrm{~m} / \mathrm{min}, 0 \% \text { incline }\end{array}$ & AIN-93M diet & $\begin{array}{l}\text { Hepatic superoxide dismutase activity, } \\
\text { mRNA expression of monocyte } \\
\text { chemotactic protein-1, percentages of } \\
\text { hepatic lipid droplets }\end{array}$ & [173] \\
\hline 5 & $\mathrm{RCT}$ & $\begin{array}{c}\text { STZ-induced diabetic Male } \\
\text { Wistar rats } \\
(n=\mathrm{NS})\end{array}$ & $45 \mathrm{~d}$ & $\begin{array}{c}\text { AB seed extracts } 200 \mathrm{mg} / \mathrm{kg} . \text { BW.day in } \\
\text { drinking water }\end{array}$ & NA & $\begin{array}{l}\text { Reduced oxidative damage by reducing } \\
\text { the expression of caspase-3, IL-6, TNF- } \alpha \\
\text { and MCP- } 1\end{array}$ & [174] \\
\hline 6 & $\mathrm{RCT}$ & Female Fischer rats $(n=32)$ & $6 \mathrm{wk}$ & $\begin{array}{l}\text { Hypercholesterolemic diet (25\% soy oil } \\
\text { and } 1 \% \text { cholesterol) supplemented with } 2 \% \\
\text { AB (dry wt/wt) }\end{array}$ & $\begin{array}{l}\text { Hypercholesterolemic diet }(25 \% \\
\text { soy oil and } 1 \% \text { cholesterol) }\end{array}$ & $\begin{array}{l}\text { Reduced expression of cholesterol } \\
\text { biosynthesis genes HMG CoA-R, EBP-2, } \\
\text { ApoB100, LDL-R, ABCG8, and CYP7A1 }\end{array}$ & [175] \\
\hline 7 & $\mathrm{RCT}$ & $\begin{array}{l}\text { STZ-induced diabetic Male } \\
\quad \text { Wistar rats }(n=40)\end{array}$ & $9 \mathrm{wk}$ & $\begin{array}{l}\mathrm{AB} \text { seed extracts } 200 \mathrm{mg} / \mathrm{kg} . \mathrm{BW} \text {.day by } \\
\text { intragastric gavage }\end{array}$ & HFD ( $55 \%$ calorie from fat) & $\begin{array}{l}\text { Hypoglycemic and hypolipidemic with } \\
\text { reduced expression of TNF- } \alpha \text { and } \\
\text { activating the insulin-signaling pathway } \\
\text { in muscle and adipose tissue }\end{array}$ & [176] \\
\hline 8 & $\mathrm{RCT}$ & $\begin{array}{l}\text { Diabetic female Fisher rats } \\
\qquad(n=\mathrm{NS})\end{array}$ & $30 \mathrm{~d}$ & $\begin{array}{l}\text { Standard AIN-93 diet supplemented with } \\
2 \%(w / w) \text { AB pulp }\end{array}$ & AIN-93 & $\begin{array}{l}\text { Modulate ROS production by } \\
\text { neutrophils and improve the liver } \\
\text { oxidant/antioxidant balance }\end{array}$ & [177] \\
\hline
\end{tabular}


Table 1. Cont.

\begin{tabular}{|c|c|c|c|c|c|c|c|}
\hline No. & Study Design & Study Subject & Duration & Berry Interventions & Intervention Diet & Significant Findings & Ref. \\
\hline \multicolumn{8}{|c|}{ (Human studies) } \\
\hline 9 & $\mathrm{CO}, \mathrm{DB}, \mathrm{RCT}$ & $\begin{array}{l}\text { Overweight healthy males } \\
\qquad(n=23)\end{array}$ & $\begin{array}{l}\text { Single day } \\
\text { meal } \\
\text { challenge }\end{array}$ & $\begin{array}{l}\text { Frozen AB pulp }(150 \mathrm{~g}) \text { was prepared in a } \\
\text { smoothie with } 50 \mathrm{~g} \text { banana }\end{array}$ & $\begin{array}{c}50 \mathrm{~g} \text { banana and matched for fat } \\
\text { with } 1.5 \mathrm{~g} \text { hexadecanoic acid } \\
\text { [palmitic acid (16:0)] and } 8.5 \mathrm{~g} \\
\text { sunflower oil [30\% } \\
\text { (9Z)-Octadec-9-enoic acid (oleic } \\
\text { acid [18:1]), 60\% } \\
\text { (9Z,12Z)-9,12-Octadecadienoic } \\
\text { acid (linoleic acid [18:2]), and } \\
10 \% \text { palmitic acid (16:0)], }\end{array}$ & $\begin{array}{l}\text { Lower incremental area under the curve } \\
\text { (iAUC) for total peroxide oxidative status } \\
\text { after açai and increased the iAUC } \\
\text { for insulin }\end{array}$ & [178] \\
\hline 10 & RCT & $\begin{array}{l}\text { Male Swiss mice } \\
\quad(n=32)\end{array}$ & $12 \mathrm{wk}$ & $\begin{array}{l}\text { A single daily dose freeze-dried AB pulp } \\
(3 \mathrm{~g} / \mathrm{kg}) \text { via gavage }\end{array}$ & $\begin{array}{l}\text { HFD ( } 32 \% \text { lard and } 1 \% \\
\text { cholesterol) }\end{array}$ & $\begin{array}{l}\text { Attenuated hepatic steatosis and reduced } \\
\text { lipid accumulation }\end{array}$ & [179] \\
\hline 11 & four-way $\mathrm{CO}$ & $\begin{array}{l}\text { Healthy men and women } \\
\qquad(n=11)\end{array}$ & $\begin{array}{l}\text { Single dose } \\
\text { study }\end{array}$ & $\begin{array}{l}100 \% \text { clarified AB juice/pulp } 7 \mathrm{~mL} / \mathrm{kg} \text { BW } \\
\text { of each study }\end{array}$ & NA & $\begin{array}{l}\text { Increased plasma antioxidant capacity } \\
\text { without affecting generation of reactive } \\
\text { oxygen species, and uric acid } \\
\text { concentrations in plasma }\end{array}$ & [180] \\
\hline 12 & $\begin{array}{l}\text { open label pilot } \\
\text { study }\end{array}$ & Overweight adults $(n=10)$ & $30 \mathrm{~d}$ & Intake of $100 \mathrm{~g}$ AB pulp twice daily & NA & $\begin{array}{l}\text { Postprandial increase in the AUC of } \\
\text { plasma glucose with reduced TC, LDL-C, } \\
\text { and LDL-C/HDL-C }\end{array}$ & [181] \\
\hline \multicolumn{8}{|c|}{ (I) Chokeberries (CB) (Animal studies) } \\
\hline 1 & $\mathrm{RCT}$ & $\begin{array}{l}\text { C57BL/6JmsSlc and KK-Ay } \\
\text { male mice }(\mathrm{N}=10, \mathrm{EACH} \\
\text { GROUP) }\end{array}$ & $4 \mathrm{wk}$ & СВ provided ad libitum & Normal chow diet & $\begin{array}{l}\text { Duction of glucose-dependent } \\
\text { insulinotropicpolypeptide (GIP) level }\end{array}$ & [182] \\
\hline 2 & $\mathrm{RCT}$ & $\begin{array}{l}\text { STZ-induced-diabetic-male } \\
\text { ICR mice }(n=32)\end{array}$ & $4 \mathrm{wk}$ & $\begin{array}{l}\text { CB extract (10-100 mg/kg.BW) daily } \\
\text { administered orally }\end{array}$ & NA & $\begin{array}{l}\text { Hypoglycemic, hypolipidemic, } \\
\text { antioxidative }\end{array}$ & [183] \\
\hline 3 & RCT & C57BL/6N mice $(n=20)$ & $12 \mathrm{wk}$ & $\begin{array}{l}\text { CB powder dissolved in water } \\
(50 \mathrm{mg} / \mathrm{kg} \text { daily })\end{array}$ & HFD (60 kcal\% Lard) & $\begin{array}{l}\text { Reduced the body and liver weight, lipid } \\
\text { accumulation, PPAR } \gamma 2 \text {, FAS, hepatic TG } \\
\text { and leptin. Serum transaminases, } \\
\text { indicators for liver antioxidant capacity } \\
\text { were significantly increased. }\end{array}$ & [184] \\
\hline 4 & $\mathrm{RCT}$ & Male C57BL/6J $(n=60)$ & $8 \mathrm{wk}$ & $\begin{array}{c}\mathrm{CB} \text { extracts }(100 \mathrm{mg} / \mathrm{kg} . \mathrm{BW}) \text { dissolved in } \\
0.5 \% \text { carboxymethyl cellulose }\end{array}$ & HFD (containing 60\% kcal fat) & $\begin{array}{l}\text { Attenuated weight-gain, increase in } \\
\text { serum TG, TC, LDL-C and better glucose } \\
\text { tolerance }\end{array}$ & [185] \\
\hline 5 & RCT & Male Wistar rats $(n=\mathrm{NS})$ & $6 \mathrm{wk}$ & $\begin{array}{l}\text { Aronia melanocarpa fruit juice (AMFJ) at } \\
\text { doses } 10 \text { and } 20 \mathrm{~mL} / \mathrm{kg}\end{array}$ & NA & Hypoglycemic, hypolipidemic & [186] \\
\hline 6 & $\mathrm{RCT}$ & Polish Merino lambs $(n=24)$ & $12 \mathrm{wk}$ & $\begin{array}{l}150-300 \mathrm{~g} \text { of chokeberry pomace per each } \\
\mathrm{kg} \text { of the complete feed mixture }\end{array}$ & Complete feed mixture & Hypoglycemic, hypolipidemic & [187] \\
\hline
\end{tabular}


Table 1. Cont

\begin{tabular}{|c|c|c|c|c|c|c|c|}
\hline No. & Study Design & Study Subject & Duration & Berry Interventions & Intervention Diet & Significant Findings & Ref. \\
\hline 7 & RCT & $\begin{array}{l}\text { Middle-aged non-medicated } \\
\text { subjects with MS }(n=38) \text { an } \\
\text { healthy volunteers }(n=14)\end{array}$ & $8 \mathrm{wk}$ & $\begin{array}{l}\text { CB extracts } 100 \mathrm{mg} / \mathrm{kg} . \mathrm{BW} \text { three } \\
\text { times daily }\end{array}$ & NA & $\begin{array}{l}\text { Beneficial changes in lipid profile, } \\
\text { coagulation parameters, inhibition of } \\
\text { platelet aggregation }\end{array}$ & [188] \\
\hline 8 & RCT & Male Wistar rats $(n=24)$ & $4 \mathrm{wk}$ & $\begin{array}{l}\text { Diet was supplemented by the extract from } \\
\text { CB fruits }(0.2 \% \mathrm{~W} / \mathrm{W}) \text { added at the expense } \\
\text { of corn starch }\end{array}$ & $\begin{array}{c}\text { Standard casein diet enriched } \\
\text { with } 0.5 \% \text { of cholesterol. Exp } \\
\text { group: the diets were modified } \\
\text { by } 8 \% \text { of lard and } 65 \% \text { of } \\
\text { fructose added at the expense } \\
\text { of soybean oil and } \\
\text { maize starch, }\end{array}$ & $\begin{array}{l}\text { Maltase and sucrase, e improvement of } \\
\text { antioxidant status, cholesterol-lowering, }\end{array}$ & [189] \\
\hline 9 & RCT & Male Wistar rats $(n=\mathrm{NS})$ & $4 \mathrm{wk}$ & CB juice $10 \mathrm{~mL} / \mathrm{kg}$. BW.day & NA & $\begin{array}{l}\text { Hypoglycemic, hypolipidemic, } \\
\text { antioxidative }\end{array}$ & [190] \\
\hline 10 & RCT & Male Wistar rats $(n=72)$ & $8 \mathrm{wk}$ & CB juice $50 \mathrm{~mL} / \mathrm{kg}$. BW.day & $\begin{array}{l}\text { High-carbohydrate, high-fat + } \\
\text { purple maize flour (HPM) }\end{array}$ & $\begin{array}{l}\text { Reduced Inflammatory cell infiltration, } \\
\text { visceral adiposity index, total body fat } \\
\text { mass, improved glucose tolerance }\end{array}$ & [191] \\
\hline 11 & RCT & Male Wistar rats $(n=36)$ & $6 \mathrm{wk}$ & CBE at 100 or $200 \mathrm{mg} / \mathrm{kg}$ BW.day & $\begin{array}{l}\text { Fructose rich diet containing } \\
\text { (g/kg diet): casein, 207; } \\
\text { DL-methionine, 3.0; fructose, } \\
\text { 600; lard, 50; cellulose, } 79 \cdot 8 ;\end{array}$ & $\begin{array}{l}\text { Elevated plasma adiponectin levels and } \\
\text { inhibited plasma TNF- } \alpha \text { and IL6. } \\
\text { Increased in the expression level of } \\
\text { glucose and lipid metabolizing genes }\end{array}$ & [192] \\
\hline 12 & $\mathrm{RCT}$ & Male Wistar albino rats $(n=60)$ & $4 \mathrm{wk}$ & $\begin{array}{l}\text { Standardized Aronia extract (SAE) } \\
0.45 \mathrm{~mL} / \mathrm{kg} . \mathrm{BW} \text { day) for } 4 \text { weeks }\end{array}$ & $\begin{array}{l}\text { HFD ( } 25 \% \text { fat, } 15 \% \text { protein, } 51 \% \\
\text { starch, and } 5 \% \text { fiber })\end{array}$ & $\begin{array}{l}\text { Reduced serum level of TC, TG, LDL-C, } \\
\text { with increased serum levels of SFA } \\
\text { and PUFA. }\end{array}$ & [193] \\
\hline \multicolumn{8}{|c|}{ (Human studies) } \\
\hline 13 & $\begin{array}{l}\text { CO open-label } \\
\text { trial }\end{array}$ & T2DM patients $(n=35)$ & $12 \mathrm{wk}$ & $\begin{array}{c}\text { Oral CB juice supplementation } \\
(150 \mathrm{~mL} / \text { day, three times a day for } 50 \mathrm{~mL})\end{array}$ & NA & $\begin{array}{l}\text { Significantly improved the renal } \\
\text { /hematological and lipid parameters (TG, } \\
\text { TC, LDL-C, LDL-C/HDL-C) in } \\
\text { diabetic patients }\end{array}$ & [194] \\
\hline 14 & $\mathrm{RCT}$ & $\begin{array}{l}\text { Healthy female volunteers } \\
\qquad(n=29)\end{array}$ & $12 \mathrm{wk}$ & $\begin{array}{l}100 \mathrm{~mL} \text { of polyphenol-rich organic CB juice } \\
\text { per day }\end{array}$ & NA & $\begin{array}{l}\text { Reduced TBARS, pro-oxidantantioxidant } \\
\text { balance, increase in paroxonase-1 activity }\end{array}$ & [195] \\
\hline 15 & RCT & $\begin{array}{l}\text { Apparently healthy women } \\
\qquad(n=25)\end{array}$ & $12 \mathrm{wk}$ & $\begin{array}{l}\text { Consume } 100 \mathrm{~mL} \text { of polyphenol-rich } \\
\text { organic } \mathrm{CB} \text { juice daily }\end{array}$ & NA & $\begin{array}{c}\text { Increased SOD and GPX activities, } \\
\text { C22:6n-3, PUFAs, total PUFAs and } \\
\text { unsaturation index and decrease in } \\
\text { n-6:n-3 ratio }\end{array}$ & [196] \\
\hline 16 & RCT & $\begin{array}{l}\text { Healthy volunteers and } \\
25 \text { patients with metabolic } \\
\text { syndrome }(n=22)\end{array}$ & $8 \mathrm{wk}$ & CB extract $(3 \times 100 \mathrm{mg} /$ day $)$ & NA & $\begin{array}{l}\text { Improvement in serum lipids, and } \\
\text { oxidative status (GSH-Px, SOD, TBARS) }\end{array}$ & [197] \\
\hline
\end{tabular}


Table 1. Cont.

\begin{tabular}{|c|c|c|c|c|c|c|c|}
\hline No. & Study Design & Study Subject & Duration & Berry Interventions & Intervention Diet & Significant Findings & Ref. \\
\hline 17 & RCT & Healthy subjects $(n=33)$ & $4 \mathrm{wk}$ & $\begin{array}{l}\text { Consume } 200 \mathrm{~mL} \text { of polyphenol-rich } \\
\text { organic CB juice daily (containing } \\
386 \pm 9.7 \mathrm{mg} \text { of total phenolics expressed } \\
\text { as gallic acid equivalents per } 100 \mathrm{~g} \text { ) }\end{array}$ & NA & $\begin{array}{l}\text { Positive effects on } \mathrm{BP} \text { and lipid status in } \\
\text { hypertensive subjects }\end{array}$ & [198] \\
\hline 18 & RCT & $\begin{array}{l}\text { Diabetic Wistar white male rats } \\
\qquad(n=48)\end{array}$ & 16 wk & $\begin{array}{c}\text { dose of polyphenols extracts } 0.040 \mathrm{~g} / \mathrm{kg} \mathrm{BW} \\
\text { every } 2 \text { day }\end{array}$ & NA & Reduced TNF- $\alpha$ and IFN- $\gamma$ levels & [199] \\
\hline 19 & RCT & $\begin{array}{l}\text { Healthy, non-smoking } \\
\text { volunteers }(n=11)\end{array}$ & $3 \mathrm{wk}$ & $\begin{array}{l}\text { CrB juice between meals ( } 250 \mathrm{~mL} \text { per day) } \\
\qquad(560 \mathrm{mg} \mathrm{GAE} / 100 \mathrm{~mL})\end{array}$ & NA & $\begin{array}{l}\text { Increased serum antioxidant capacity } \\
\text { with no significant change in the blood } \\
\text { lipid profile }\end{array}$ & [200] \\
\hline 20 & RCT & $\begin{array}{l}\text { Men with the diagnosed mild } \\
\text { hypercholesterolemia }(n=58)\end{array}$ & $6 \mathrm{wk}$ & $\begin{array}{l}\text { CB juice between meals ( } 250 \mathrm{~mL} \text { per day) } \\
\text { (560 mg GAE/100 mL) }\end{array}$ & NA & $\begin{array}{c}\text { Improved lipid profile with reduced } \\
\text { lipid peroxides (LPO), C-reactive high } \\
\text { sensitivity protein (hsCRP), } \\
\text { homocysteine, }\end{array}$ & [201] \\
\hline 21 & $\begin{array}{l}\text { 3-arm, DB, } \\
\text { parallel RCT }\end{array}$ & $\begin{array}{l}\text { Healthy male volunteers } \\
\qquad(n=66)\end{array}$ & $12 \mathrm{wk}$ & $\begin{array}{c}\text { CB extract" capsules (containing } 116 \mathrm{mg} \\
\text { total (poly)phenols). CB whole fruit" } \\
\text { capsules (containing the equivalent to } 10 \mathrm{~g} \\
\text { of the whole CB fruit, and } 12 \mathrm{mg} \text { of total } \\
\text { (poly)phenols) }\end{array}$ & NA & $\begin{array}{l}\text { Increased Anaerostipes, Bifidobacterium, } \\
\text { Faecalibacterium, and Clostridium genera }\end{array}$ & [202] \\
\hline \multicolumn{8}{|c|}{ (I) Black Currants (BCT) (Animal studies) } \\
\hline 1 & RCT & $\begin{array}{l}\text { Old male Sprague-Dawley } \\
\text { (SD) rats }(n=\mathrm{NS})\end{array}$ & $\begin{array}{l}\text { Single meal } \\
\text { challenge } \\
\text { test }\end{array}$ & BCE 5 mg/kg.BW (1 mg D3R/kg.BW) & $\begin{array}{l}\text { Normal diet with IP } \\
\text { administration of glucose } \\
\text { solution }(2 \mathrm{~g} / \mathrm{kg})\end{array}$ & $\begin{array}{l}\text { Improved hyperglycemic and } \\
\text { hypoinsulinemic condition }\end{array}$ & [203] \\
\hline 2 & RCT & Male KK-Ay $(n=16)$ & $7 \mathrm{wk}$ & $\begin{array}{c}\text { BC extracts ( } 2 \mathrm{~g} / \mathrm{Kg} \text {.diet) (equivalent to } \\
\text { delphinidine-3-glucoside (D3R) } \\
2 \mathrm{~g} / \mathrm{Kg} \text {.diet) }\end{array}$ & NA & $\begin{array}{l}\text { Improved glucose tolerance with } \\
\text { increased GLP-1 concentration, and } \\
\text { upregulation of AMPK- } \alpha \text { and } \\
\text { prohormone convertase } \\
\text { 1/3(GLP-1 precursor) }\end{array}$ & [204] \\
\hline 3 & $\mathrm{RCT}$ & Male C57BL/6J mice $(n=48)$ & $8 \mathrm{wk}$ & $\begin{array}{l}\text { Diet supplemented with } 1 \% \mathrm{BC} \text { powdered } \\
\text { extract ( } 32 \% \text { anthocyanins) }\end{array}$ & HFD (60 kcal\% fat diet) & $\begin{array}{l}\text { Protective effect of } \mathrm{BC} \text { anthocyanins } \\
\text { against obesity and associated } \\
\text { insulin resistance. }\end{array}$ & [205] \\
\hline 4 & RCT & Male C57BL/6J mice $(n=24)$ & $12 \mathrm{wk}$ & $\begin{array}{l}\mathrm{HF} / \mathrm{HC} \text { diet supplemented with } 0.1 \% \text { of } \\
\mathrm{BCE} \text { (containing } 25 \% \text { anthocyanins and } \\
40 \% \text { polyphenols) by weight }\end{array}$ & $\begin{array}{l}\text { AIN-93M high fat/high } \\
\text { cholesterol (HF/HC) diet }(16 \% \\
\text { fat, } 0.25 \% \text { cholesterol by weight; } \\
55.7 \%, 125.5 \% \text { and } 31.8 \% \text { energy } \\
\text { from carbohydrate, protein and } \\
\text { fat, respectively; } 4529 \mathrm{kcal} / \mathrm{Kg}\end{array}$ & $\begin{array}{l}\text { Reduced BW and adipocyte size of the } \\
\text { epididymal fat, energy expenditure and } \\
\text { mitochondrial biogenesis genes }\end{array}$ & [206] \\
\hline
\end{tabular}


Table 1. Cont

\begin{tabular}{|c|c|c|c|c|c|c|c|}
\hline No. & Study Design & Study Subject & Duration & Berry Interventions & Intervention Diet & Significant Findings & Ref. \\
\hline 5 & RCT & $\begin{array}{l}\text { Male New Zealand white } \\
\text { rabbits }(n=20)\end{array}$ & $4 \mathrm{wk}$ & $\begin{array}{l}\text { Diet supplemented with } 1.5 \% \text { BC } \\
\text { polyphenolic extract }\end{array}$ & $\begin{array}{l}\text { HFD }(10 \% \text { lard }) \text { was } 17 \% \text { from } \\
\text { protein, } 32 \% \text { from fat and } 51 \% \\
\text { from carbohydrates }\end{array}$ & $\begin{array}{c}\text { Reduced concentration of putrefactive } \\
\text { metabolites, } \beta \text {-glucuronidase activity, } \\
\text { ameliorated hyperlipidemia, and } \\
\text { antioxidative capacity }\end{array}$ & [207] \\
\hline 6 & RCT & $\begin{array}{l}\text { Sprague-Dawley male rats } \\
\qquad(n=40)\end{array}$ & $4 \mathrm{wk}$ & $\begin{array}{c}2 \mathrm{~mL} \text { of BC extract (containing } 30 \mathrm{mg} \text { BC } \\
/ \mathrm{kg} \mathrm{BW} \text { ) or } 2 \mathrm{~mL} \text { of CAM30 extract } \\
\text { (containing } 13.4 \mathrm{mg} \text { CAM30/kg body } \\
\text { weight), respectively, three times weekly } \\
\text { by oral gavage }\end{array}$ & NA & $\begin{array}{l}\text { Reduced } \beta \text {-glucuronidase activity and } \\
\text { undesirable bacteria in the caeca. } \\
\text { Increased lactobacilli and bifidobacterial } \\
\text { gut species }\end{array}$ & [208] \\
\hline 7 & RCT & $\begin{array}{l}\text { Male Sprague-Dawley (SD) } \\
\text { rats }(n=40)\end{array}$ & $8 \mathrm{wk}$ & $\begin{array}{l}\text { BC extract } 100-300 \mathrm{mg} / \mathrm{kg} . \text { BW.day } \\
\text { administered orally }\end{array}$ & $\begin{array}{l}\text { High-fructose (HF) diet }(60 \% \\
\text { fructose diet) }\end{array}$ & $\begin{array}{l}\text { Improvements in hypertension, } \\
\text { dyslipidemia, insulin resistance, } \\
\text { and obesity }\end{array}$ & [209] \\
\hline 8 & RCT & $\begin{array}{l}\text { Male Sprague-Dawley rats } \\
\qquad(n=128)\end{array}$ & $6 \mathrm{wk}$ & $\begin{array}{c}\text { Diets with dietary fiber and BC extracts } \\
\text { (Currantex } 30)(\text { containing total } \\
\text { anthocyanin } 32 \%(w / w))\end{array}$ & NA & $\begin{array}{l}\text { Increased intestinal population of SCFA } \\
\text { and total beneficial bacterial population }\end{array}$ & [210] \\
\hline \multicolumn{8}{|c|}{ (Human studies) } \\
\hline 9 & $\mathrm{RCT}$ & Healthy volunteers $(n=30)$ & $2 \mathrm{wk}$ & $\begin{array}{c}\text { BC extracts }(1500 \mathrm{mg} / \text { day; } \\
375 \mathrm{mg} \times 4 \text { capsules }) \\
\text { BC powder CAM30 }(672 \mathrm{mg} / \text { day; } \\
168 \mathrm{mg} \times 4 \text { capsules })\end{array}$ & NA & $\begin{array}{l}\text { Increased intestinal population of SCFA } \\
\text { and total beneficial bacterial population }\end{array}$ & [211] \\
\hline 10 & $\mathrm{DB}, \mathrm{CO}, \mathrm{RCT}$ & Healthy subjects $(n=26)$ & $\begin{array}{l}\text { Single meal } \\
\text { challenge } \\
\text { test }\end{array}$ & $\begin{array}{l}\text { Apple and BC polyphenol-rich drinks } \\
\text { (1200 mg apple polyphenols (AE), } \\
\text { or } 600 \mathrm{mg} \text { apple polyphenols }+600 \mathrm{mg} \mathrm{BC} \\
\text { anthocyanins (AE+BE)) }\end{array}$ & $\begin{array}{l}\text { Standardized } \\
\text { high-carbohydrate meal } 100 \mathrm{~g} \\
\text { of white bread }\end{array}$ & $\begin{array}{l}\text { Reduced Postprandial insulin, C-peptide } \\
\text { and GIP, GLUT and SGLT1-mediated } \\
\text { glucose transport }\end{array}$ & [212] \\
\hline 11 & $\mathrm{DB}, \mathrm{CO}, \mathrm{RCT}$ & Healthy subjects $(n=22)$ & $\begin{array}{l}\text { Single meal } \\
\text { challenge } \\
\text { test }\end{array}$ & $\begin{array}{l}\text { Low-sugar-BC drink containing } \\
\quad 300-600 \mathrm{mg} \text { anthocyanins }\end{array}$ & $\begin{array}{c}\text { Standardized } \\
\text { high-carbohydrate meal } 100 \mathrm{~g} \\
\text { of white bread }\end{array}$ & $\begin{array}{l}\text { Reduced postprandial insulinemia, } \\
\text { glycemia, and incretin secretion }\end{array}$ & [213] \\
\hline 12 & RCT & Healthy participants $(n=17)$ & $6 \mathrm{~d}$ & $\mathrm{BC}$ powder $6 \mathrm{~g} /$ day with water & NA & $\begin{array}{l}\text { Improved postprandial AUC of glucose } \\
\text { and insulin }\end{array}$ & [214] \\
\hline 13 & $\mathrm{DB}, \mathrm{CO}, \mathrm{RCT}$ & $\begin{array}{l}\text { Endurance-trained females } \\
\qquad(n=16)\end{array}$ & $7 \mathrm{~d}$ & BC extract $600 \mathrm{mg} /$ day & NA & Increased fat oxidation & [215] \\
\hline 14 & RCT & $\begin{array}{l}\text { Healthy sedentary male and } \\
\text { female participants }(n=40)\end{array}$ & $\begin{array}{l}\text { Single meal } \\
\text { challenge } \\
\text { test }\end{array}$ & BC juice $200 \mathrm{~mL} /$ participant & $\begin{array}{l}\text { standardized meal bar to } \\
\text { consume for breakfast at least } \\
1 \mathrm{~h} \text { prior to starting } \\
\text { the trial. }\end{array}$ & Supported positive affective responses & [216] \\
\hline 15 & $\begin{array}{c}\text { parallel, } \\
\text { four-arm, study } \\
\text { design + DB, CO } \\
\text { parallel trial }\end{array}$ & $\begin{array}{l}\text { Healthy individuals }(n=24) \\
\qquad(n=32)\end{array}$ & $\begin{array}{l}\text { A single } \\
\text { meal } \\
\text { challenge } \\
\text { study }\end{array}$ & $\begin{array}{c}\text { Two opaque gelatin capsules containing } \\
\text { BC anthocyanin }(3.2 \mathrm{mg} / \mathrm{kg} \text { total } \\
\text { anthocyanins })\end{array}$ & NA & $\begin{array}{l}\text { Dose-dependent increase in plasma } \\
\text { anthocyanins and recovery from } \\
\text { exercise-induced oxidative stress }\end{array}$ & [217] \\
\hline
\end{tabular}


Table 1. Cont.

\begin{tabular}{|c|c|c|c|c|c|c|c|}
\hline No. & Study Design & Study Subject & Duration & Berry Interventions & Intervention Diet & Significant Findings & Ref. \\
\hline \multicolumn{8}{|c|}{ (I) Maqui berries (MqB) (Animal studies) } \\
\hline 1 & $\begin{array}{l}\text { open exploratory } \\
\text { study }\end{array}$ & Pre-diabetic volunteers $(n=43)$ & $\begin{array}{l}\text { Single dose } \\
\text { study }\end{array}$ & $\begin{array}{c}\text { A single dose of Delphinol Capsules } \\
\text { bearing either 60, 120, or } 180 \mathrm{mg} \text { Delphinol } \\
\text { on each day with one-week } \\
\text { washout period }\end{array}$ & NA & $\begin{array}{l}\text { Dose dependently lowered basal } \\
\text { insulinemia and glycemia }\end{array}$ & [218] \\
\hline 2 & $\mathrm{RCT}$ & Male balb/c mice $(n=\mathrm{NS})$ & $7 \mathrm{~d}$ & MqB extracts $(25,50$ and $100 \mathrm{mg} / \mathrm{kg} . \mathrm{BW})$ & NA & Ameliorate the oxidative stress condition & [219] \\
\hline 3 & RCT & Male C57BL/BJ mice & $12 \mathrm{wk}$ & $\begin{array}{c}\text { MqB anthocyanins (ANC), Labrasol/water: } \\
66 / 34+\text { ANC (LAB + ANC) }\end{array}$ & $\operatorname{HFD}(60 \%$ calories from fat $)$ & $\begin{array}{c}\text { Decreased glucose production, } \\
\text { down-regulation of gluconeogenic } \\
\text { enzyme }\end{array}$ & [220] \\
\hline 4 & $\mathrm{DB}, \mathrm{CO}, \mathrm{RCT}$ & $\begin{array}{l}\text { Fifty overweight volunteer } \\
\text { smokers }(n=42)\end{array}$ & $4 \mathrm{wk}$ & $\begin{array}{l}3 \text { capsules of } 150 \mathrm{mg} \text { standardized maqui } \\
\text { berry extract containing } 54 \mathrm{mg} \text { of } \\
\text { anthocyanin daily (equivalent to } 162 \mathrm{mg} \\
\text { anthocyanins/day) }\end{array}$ & NA & $\begin{array}{l}\text { Reduced levels of Ox-LDL in the } \\
\text { anthocyanin group }\end{array}$ & [221] \\
\hline 5 & RCT & $\begin{array}{l}\text { Male C57BL/6Nhsd mice } \\
\qquad(n=18)\end{array}$ & $4 \mathrm{wk}$ & $\begin{array}{l}\text { MqB derived Delphinidine } \\
(15 \mathrm{mg} / \mathrm{kg} . \text { BW }) \text { daily }\end{array}$ & $\begin{array}{l}\text { High-fat diet and } \\
\text { high-carbohydrate drinking } \\
\text { water ( } 45 \% \text { kcal from fat) }\end{array}$ & $\begin{array}{l}\text { Reduced TG accumulation with no effect } \\
\text { on metabolic alterations related glucose } \\
\text { metabolism }\end{array}$ & [222] \\
\hline 6 & $\begin{array}{l}\text { Prospective } \\
\text { observational } \\
\text { study }\end{array}$ & $\begin{array}{l}\text { Middle-aged participants } \\
\qquad(n=21)\end{array}$ & $8 \mathrm{wk}$ & $\begin{array}{l}\text { Two tablets per day of an MCN (Eonlipid) } \\
\text { (containing maqui, } 300 \mathrm{mg} \text { in each tablet) }\end{array}$ & NA & $\begin{array}{l}\text { Improvement of most atherogenesis and } \\
\text { oxidative stress biomarkers }\end{array}$ & [223] \\
\hline 7 & $\mathrm{CO}, \mathrm{RCT}$ & Healthy male subjects $(n=11)$ & ONCS & $\begin{array}{l}\text { Intake of } 250 \mathrm{~mL} \text { of the MqB drink } \\
\text { containing an number of total polyphenols } \\
\sim 1000 \mu \mathrm{mol} \text { equivalents of gallic acid }\end{array}$ & $\begin{array}{c}\text { Meals containing food-grade } \\
\text { glucose and rice, containing } \\
50 \mathrm{~g} \text { of carbohydrates by each } \\
\text { meal }\end{array}$ & $\begin{array}{l}\text { Reduced glycemic indexed for } \\
\text { high-carbohydrate diets. }\end{array}$ & [224] \\
\hline 8 & RCT & $\begin{array}{l}\text { C57BL/6J littermates' male } \\
\text { mice }(n=23)\end{array}$ & $16 \mathrm{wk}$ & $\begin{array}{l}\text { HFD supplemented with } 4-5 \mathrm{mg} \text { of MqB } \\
\text { polyphenols/ } 10-15 \mathrm{kcal} \text { per day }\end{array}$ & $\operatorname{HFD}(45 \%$ calories from fat) & $\begin{array}{l}\text { Reduced body-weight-gain, improved } \\
\text { glucose tolerance and insulin resistance. } \\
\text { Differential expression of genes involved } \\
\text { in fatty acid oxidation, de novo } \\
\text { lipogenesis, thermogenesis, and } \\
\text { multilocular lipid droplet formation }\end{array}$ & [225] \\
\hline \multicolumn{8}{|c|}{$\begin{array}{l}\text { Note: Acai berry, AB; AB juice, ABJ; ATP-binding cassette sub-family G member 8, ABCG8; AMP-activated-proteins kinase- } \alpha \text {, AMPK- } \alpha \text {; AB extracts, ABE; ATP-binding cassette sub-family G } \\
\text { member 5, ABCG5; vascular cell adhesion molecule-1 VCAM-1; Apolipoprotein B, ApoB; Area under curve, AUC; Bilberry, BB; BB juice, BBJ; BB extracts, BBE; Black currant, BCT; BCT juice, } \\
\text { BCTJ, BCT extracts, BCTE; Blueberry, BlB; BlB juice BlBJ; BlB extracts, BlBE; body weight, BW; C-reactive high sensitivity protein hsCRP; catalase CAT; Cytochrome P450 Family 7 Subfamily } \\
\text { A Member 1, CYP7A1; Chokeberry, CB; cranberry, CrB; Cross-over, CO; cross-sectional, CS; cyanidin-3-O-glucoside, C3G; day, d; Double-blind, DB; Freeze-dried, F/D; High-fat-diet, HFD; } \\
\text { low-fat-diet, LFD; glucose transporter 1, GLT; glucagon-like-peptide 1, GLP1; Glutathione peroxidase GPx; glutathione reductase GSH-x; 3-hydroxy-3-methylglutaryl-CoA, HMG-CoA; } \\
\text { interferon alpha IFN- } \alpha \text {; Intercellular Adhesion Molecule 1, ICAM-1; interleukin, IL; Lingonberry, LB; Low-fat-diet, LFD; Low-Density Lipoprotein (LDL) Receptor (LDL-R); Monocyte } \\
\text { Chemoattractant Protein } 1 \text { (MCP-1); Mulberry, MB; Maqui berry, MqB; Ox-LDL, nonalcoholic fatty liver disease (NAFLD); oxidized low-density-lipoproteins; oxLDL-C; polyunsaturated } \\
\text { fatty acids, PUFA; thiobarbituric acid reactive substances (TBARS); total glyceraldehyde, TG; total cholesterol, TC; Tumor necrosis factor, TNF- } \alpha \text {; single-blinded, SB; Superoxide dismutase, } \\
\text { sOD; one-time-challenge-study, OTCS; placebo-controlled, PC; Peroxisome proliferator-activated receptor- } \alpha \text {, PPAR } \alpha \text {; phosphoenolpyruvate carboxykinase (Pepck); Raspberry, RB; } \\
\text { Randomized controlled trial, RCT; respiratory quotient (RQ), short-chain fatty acids, SCFA; sodium glucose transporter protein, SGLT; Sterol regulatory element-binding protein, SREBP-1c; } \\
\text { weeks, wk. }\end{array}$} \\
\hline
\end{tabular}


Table 2. A comprehensive list of potential health promoting individual anthocyanins and phenolic compounds with their quantities found in berries or berry products.

\begin{tabular}{|c|c|c|c|c|c|c|c|c|c|c|c|c|c|}
\hline Compounds & $\begin{array}{l}\text { Bilberry } \\
\text { (mg/100 } \\
\mathrm{g} \mathrm{fw})\end{array}$ & $\begin{array}{c}\text { Blueberry } \\
\text { (mg/100 } \\
\mathrm{g} \text { fw) }\end{array}$ & $\begin{array}{c}\text { Cranberry } \\
(\mathrm{mg} / 100 \\
\mathrm{g} \text { fw) }\end{array}$ & $\begin{array}{c}\text { Raspberry } \\
(\mathrm{mg} / 100 \\
\mathrm{g} \mathrm{dw})\end{array}$ & $\begin{array}{c}\text { Mulberry } \\
\text { (mg/100 } \\
\mathrm{g} \mathrm{fw})\end{array}$ & $\begin{array}{l}\text { Lingonberry } \\
\text { (mg/g DE) }\end{array}$ & $\begin{array}{l}\text { Blackberry } \\
\text { (mg/g DE) }\end{array}$ & $\begin{array}{c}\text { Strawberry } \\
(\mathrm{mg} / 100 \\
\mathrm{g} \mathrm{fw})\end{array}$ & $\begin{array}{c}\text { Goji Berry } \\
\text { (mg/100 } \\
\mathrm{g} \mathrm{dw})\end{array}$ & $\begin{array}{c}\text { Acai Berry } \\
\text { (mg/100 } \\
\mathrm{g} \mathrm{dw})\end{array}$ & $\begin{array}{c}\text { Black } \\
\text { Chokeberry } \\
(\mathrm{mg} / 100 \mathrm{~g} \\
\mathrm{dw})\end{array}$ & $\begin{array}{c}\text { Black } \\
\text { Currant } \\
(\mathrm{mg} / 100 \mathrm{~g} \\
\mathrm{fw})\end{array}$ & $\begin{array}{l}\text { Maqui Berry } \\
\text { (mg/100 g } \\
\text { fw) }\end{array}$ \\
\hline References & {$[38,51]$} & [10,35-39] & [226-229] & [230-232] & [233-235] & {$[236,237]$} & {$[131,238,239]$} & {$[240,241]$} & {$[62,123,242,243]$} & [244-246] & [247] & {$[248,249]$} & {$[219,250,251]$} \\
\hline Cyanidin & $18-290$ & - & - & - & - & - & - & $27-175$ & 27.5 & - & - & - & $22.8-26.0$ \\
\hline Delphinidin & $29-280$ & - & - & - & - & - & - & - & - & - & - & - & $105.0-120.3$ \\
\hline Quercetin & $1.5-8$ & 0.07 * & 104 & - & $0.3-10.04$ & - & - & $0.09-0.54$ & - & 39.02 & $37-400$ & - & - \\
\hline Myricetin & nd-3 & - & 69 & - & & - & - & $0.05-0.77$ & - & - & - & - & - \\
\hline$p$-Coumaric acid & $1-9$ & - & 0.25 & $67.03-2792.6$ & $0.3-4.2$ & 0.13 & - & 2.64 & $0.07-0.22$ & - & - & - & - \\
\hline$m$-Coumaric acid & $7-30$ & - & - & - & $0.3-14.2$ & - & 0.93 & - & - & - & - & - & - \\
\hline Sinapic acid & - & - & 0.211 & - & & - & - & 0.61 & - & - & - & - & - \\
\hline Gallic acid & - & - & - & $3-72.2$ & $3.8-8.6$ & - & - & $26.5-47.54$ & - & 701.6 & - & - & 75 \\
\hline Ascorbic acid & - & - & 0.011 & $2.4-5.34$ & & - & - & - & - & - & - & - & - \\
\hline Ferulic acid & - & - & 0.087 & - & $5.3-294$ & - & - & 0.95 & 753.6 & 2.46 & - & - & - \\
\hline Chlorogenic acid & - & $3.08^{*}$ & - & 177.4 & $4.3-22.3$ & - & - & $0.35-1.10$ & - & 37.65 & - & - & - \\
\hline Protocatechuic acid & - & - & - & - & 3 & - & - & - & - & - & - & - & - \\
\hline $\begin{array}{l}\text { 5-O-Caffeoylquinic } \\
\text { Acid }\end{array}$ & - & - & - & - & 283-1735 & - & $5.57-8.88$ & - & $8.4-37.9$ & - & $346-413$ & - & - \\
\hline $\begin{array}{l}\text { 1,3-di-O-Caffeoylquinic } \\
\text { Acid }\end{array}$ & - & - & - & - & $0.2-0.3$ & - & $0.15-0.22$ & - & $0.6-4.27$ & - & $13-508$ & - & - \\
\hline Caffeic acid & - & - & 0.15 & $2.41-5.31$ & $1.3-9.2$ & 0.26 & - & 0.52 & $0.76-1.52$ & 8.12 & - & - & - \\
\hline Protocatechuic acid & $4-8$ & - & - & - & & - & - & - & - & - & - & - & - \\
\hline Ellagic acid & - & - & 120 & 1151.7 & 23.9 & - & 2.012 & 2.72 & - & - & - & - & - \\
\hline Benzoic acid & - & - & 4.7 & - & - & 3.79 & - & - & - & - & - & - & - \\
\hline $\begin{array}{l}p \text {-Hydroxyphenylacetic } \\
\text { acid }\end{array}$ & - & - & 0.007 & - & $4.3-12.9$ & - & - & - & - & - & - & - & - \\
\hline $\begin{array}{l}\text { 2,3-Dihydroxybenzoic } \\
\text { acid }\end{array}$ & - & - & 0.003 & - & 12.9 & - & - & - & - & 28.18 & - & - & - \\
\hline $\begin{array}{l}\text { 2,4-Dihydroxy benzoic } \\
\text { acid }\end{array}$ & - & - & 0.04 & - & - & - & - & - & $0.13-0.51$ & 3.37 & - & - & - \\
\hline Vanillic acid & - & - & 0.05 & $3-4.41$ & - & - & - & $2.91-3.1$ & $4-6.37$ & 57.7 & - & - & - \\
\hline Trans-cinnamic acid & - & - & 0.02 & - & - & - & - & - & - & - & - & - & - \\
\hline $\begin{array}{l}\text { O-Hydroxycinnamic } \\
\text { acid }\end{array}$ & - & - & 0.089 & - & - & - & - & - & - & - & - & - & - \\
\hline$p$-Hydroxybenzoic acid & - & - & 0.021 & - & - & - & - & - & - & 172 & - & - & - \\
\hline Resveratrol & $1-12$ & - & - & - & - & 0.13 & - & - & - & - & - & - & - \\
\hline Epigallocatechin & - & - & 1.5 & - & 25.6 & - & - & - & - & - & - & - & - \\
\hline$(+/-)$-Catechins & $6-7$ & - & 4.5 & 129.3 & - & - & - & $19.56-135.19$ & 106.6 & 49.1 & 593 & - & - \\
\hline$(+/-)$ Epicatechin & $6-7$ & - & 4.5 & 791.7 & $0.2-24$ & - & - & 1.07 & - & 44.6 & 6767 & - & - \\
\hline Gallocatechin gallate & - & - & 0.4 & - & $10.2-63.7$ & - & - & - & - & - & - & - & - \\
\hline $\begin{array}{l}\text { Epigallocatechin } \\
\text { gallate }\end{array}$ & - & - & 1.9 & - & $4.5-8.4$ & - & - & 5.65 & - & - & - & - & - \\
\hline $\begin{array}{l}\text { Delphinidin } \\
\text { 3-galactoside }\end{array}$ & 167.1 & 23.4 & - & - & - & & - & - & - & - & - & $52^{* * *}$ & - \\
\hline $\begin{array}{l}\text { Delphinidin } \\
\text { 3-glucoside }\end{array}$ & 169.1 & 15.4 & - & - & - & & $26.8-29.40$ & - & - & - & - & $839^{* * *}$ & 389.9 \\
\hline
\end{tabular}


Table 2. Cont.

\begin{tabular}{|c|c|c|c|c|c|c|c|c|c|c|c|c|c|}
\hline Compounds & $\begin{array}{l}\text { Bilberry } \\
\text { (mg/100 } \\
\mathrm{g} \mathrm{fw})\end{array}$ & $\begin{array}{l}\text { Blueberry } \\
\text { (mg/100 } \\
\text { g fw) }\end{array}$ & $\begin{array}{l}\text { Cranberry } \\
(\mathrm{mg} / 100 \\
\mathrm{g} \mathrm{fw})\end{array}$ & $\begin{array}{c}\text { Raspberry } \\
\text { (mg/100 } \\
\text { gdw) }\end{array}$ & $\begin{array}{c}\text { Mulberry } \\
\text { (mg/100 } \\
\text { g fw) }\end{array}$ & $\begin{array}{l}\text { Lingonberry } \\
\text { (mg/g DE) }\end{array}$ & $\begin{array}{l}\text { Blackberry } \\
\text { (mg/g DE) }\end{array}$ & $\begin{array}{c}\text { Strawberry } \\
(\mathrm{mg} / 100 \\
\mathrm{g} \mathrm{fw})\end{array}$ & $\begin{array}{c}\text { Goji Berry } \\
\text { (mg/100 } \\
\text { g dw) }\end{array}$ & $\begin{array}{c}\text { Acai Berry } \\
(\mathrm{mg} / 100 \\
\mathrm{g} \mathrm{dw})\end{array}$ & $\begin{array}{c}\text { Black } \\
\text { Chokeberry } \\
\text { (mg/100 g } \\
\text { dw) }\end{array}$ & $\begin{array}{c}\text { Black } \\
\text { Currant } \\
\text { (mg/100 g } \\
\text { fw) }\end{array}$ & $\begin{array}{l}\text { Maqui Berry } \\
\text { (mg/100 g } \\
\text { fw) }\end{array}$ \\
\hline Cyanidin 3-galactoside & 122.6 & 4.2 & 2 & - & - & & - & - & - & - & $105-2407$ & - & - \\
\hline $\begin{array}{l}\text { Delphinidin } \\
\text { 3-arabinoside }\end{array}$ & 152.3 & - & - & - & - & & - & - & - & - & - & - & - \\
\hline Cyanidin 3-glucoside & 130.4 & 2.6 & 0.1 & $15.02-53.94$ & 4.72 & & - & - & - & 998.74 & $5-113$ & $327^{* *}$ & 679 \\
\hline $\begin{array}{l}\text { Petunidin } \\
\text { 3-galactoside }\end{array}$ & 50 & 11.7 & - & - & - & & - & - & - & - & - & $103^{* * *}$ & - \\
\hline Cyanidin 3-arabinoside & 110.6 & 3.5 & 1.4 & - & - & & - & - & - & - & $215-1148$ & - & - \\
\hline Petunidin 3-glucoside & 101.9 & 12.4 & - & - & - & & $10.02-15.25$ & - & - & 21 & - & - & - \\
\hline Peonidin 3-galactoside & 13.3 & 1.8 & 2.8 & - & - & & - & - & - & - & - & - & - \\
\hline $\begin{array}{c}\text { Petunidin } \\
\text { 3-arabinoside }\end{array}$ & 23.9 & 9.3 & - & - & - & & - & - & - & - & - & - & - \\
\hline Peonidin 3-glucoside & 56.7 & 2.1 & 0.3 & - & - & & $2.04-3.62$ & - & - & 193 & - & $71^{* * *}$ & - \\
\hline Malvidin 3-galactoside & 27.5 & 34.9 & - & - & - & & - & - & - & - & - & - & - \\
\hline Peonidin 3-arabinoside & 4.5 & 1 & 1.1 & - & - & & - & - & - & - & - & - & - \\
\hline Malvidin 3-glucoside & 67.7 & 31.2 & - & - & - & & $9.49-10.57$ & - & - & - & - & - & - \\
\hline Malvidin 3-arabinoside & 12.8 & 34.7 & - & - & - & & - & - & - & - & - & - & - \\
\hline $\begin{array}{l}\text { Quercetin-3- } \\
\text { galactoside }\end{array}$ & - & - & 70.4 & - & - & & - & - & - & - & - & - & - \\
\hline $\begin{array}{l}\text { Quercetin-3- } \alpha- \\
\text { arabinopyranoside }\end{array}$ & - & - & 34.4 & - & - & & - & - & - & - & - & - & - \\
\hline Quercetin-3-rhamnoside & - & - & 41.6 & - & - & & - & - & - & - & - & - & - \\
\hline Kaempferol-3-glucoside & - & - & 5.6 & - & - & & - & $5.12-17.67$ & - & - & - & - & - \\
\hline $\begin{array}{l}\text { Myricetin 3- } \alpha- \\
\text { arabinofuranoside }\end{array}$ & - & - & 37.5 & - & - & & - & - & - & - & - & - & - \\
\hline $\begin{array}{l}\text { Quercetin } \\
\text { 3-O-glucuronide }\end{array}$ & - & - & - & 717.57 & - & & - & $9.4-39$ & - & - & - & - & - \\
\hline Quercetin pentoside & - & - & - & 252 & - & & - & - & - & - & - & - & - \\
\hline $\begin{array}{l}\text { Cyanidin-3-O- } \\
\text { sophoroside }\end{array}$ & - & - & - & $43.27-800.3$ & - & & - & - & - & - & - & - & - \\
\hline $\begin{array}{l}\text { Cyanidin-3-O- } \\
\text { rutinoside }\end{array}$ & - & - & - & $5.49-104.58$ & 2.73 & & - & - & - & 433.98 & - & $1693^{* * *}$ & - \\
\hline $\begin{array}{l}\text { Pelargonidin-3- } \\
\text { glucoside }\end{array}$ & - & - & - & - & 0.14 & & - & - & - & - & - & - & - \\
\hline $\begin{array}{l}\text { Quercetin } \\
\text { 3-O-rutinoside }\end{array}$ & - & - & - & - & $192-398$ & & - & - & $0.9-23.2$ & - & - & $1.8-2.37$ & - \\
\hline $\begin{array}{l}\text { Quercetin } \\
\text { 3-O-galactoside }\end{array}$ & - & - & - & - & $0.2-345$ & & - & - & - & - & - & - & - \\
\hline $\begin{array}{c}\text { Quercetin } \\
\text { 3-O-glucoside }\end{array}$ & - & - & - & - & $72.4-345.7$ & & $0.23-0.88$ & $9.8-25.1$ & $16.9-90.9$ & 44-3756 & - & $1.5-2.0$ & - \\
\hline $\begin{array}{l}\text { Kaempferol } \\
\text { 3-O-glucoside }\end{array}$ & - & - & - & - & $35.5-478$ & & - & $5.96-14.39$ & $0.5-1.94$ & - & - & - & - \\
\hline $\begin{array}{l}\text { Pelargonidin } \\
\text { 3-O-rutinoside }\end{array}$ & - & - & - & - & $17.8-290$ & & - & - & - & - & - & - & - \\
\hline
\end{tabular}


Table 2. Cont

\begin{tabular}{|c|c|c|c|c|c|c|c|c|c|c|c|c|c|}
\hline Compounds & $\begin{array}{c}\text { Bilberry } \\
(\mathrm{mg} / 100 \\
\mathrm{g} \mathrm{fw})\end{array}$ & $\begin{array}{c}\text { Blueberry } \\
\text { (mg/100 } \\
\mathrm{g} \mathrm{fw})\end{array}$ & $\begin{array}{c}\text { Cranberry } \\
\text { (mg/100 } \\
\mathrm{g} \mathrm{fw})\end{array}$ & $\begin{array}{c}\text { Raspberry } \\
\text { (mg/100 } \\
\text { g dw) }\end{array}$ & $\begin{array}{c}\text { Mulberry } \\
\text { (mg/100 } \\
\mathrm{g} \mathrm{fw})\end{array}$ & $\begin{array}{l}\text { Lingonberry } \\
\text { (mg/g DE) }\end{array}$ & $\begin{array}{l}\text { Blackberry } \\
\text { (mg/g DE) }\end{array}$ & $\begin{array}{c}\text { Strawberry } \\
(\mathrm{mg} / 100 \\
\mathrm{g} \mathrm{fw})\end{array}$ & $\begin{array}{c}\text { Goji Berry } \\
\text { (mg/100 } \\
\text { g dw) }\end{array}$ & $\begin{array}{c}\text { Acai Berry } \\
\text { (mg/100 } \\
\mathrm{g} \mathrm{dw})\end{array}$ & $\begin{array}{c}\text { Black } \\
\text { Chokeberry } \\
(\mathrm{mg} / 100 \mathrm{~g} \\
\mathrm{dw})\end{array}$ & $\begin{array}{c}\text { Black } \\
\text { Currant } \\
\text { (mg/100 g } \\
\text { fw) }\end{array}$ & $\begin{array}{l}\text { Maqui Berry } \\
\text { (mg/100 g } \\
\text { fw) }\end{array}$ \\
\hline $\begin{array}{c}\text { Delphinidin-O- } \\
\text { (pentosyl)hexoside }\end{array}$ & - & - & - & - & - & - & $0.82-1.88$ & - & - & - & - & - & - \\
\hline $\begin{array}{l}\text { Delphinidin-O- } \\
\text { rhamnoside }\end{array}$ & - & - & - & - & - & - & 2.14 & - & - & - & - & - & - \\
\hline Malvidin-O-pentoside & - & - & - & - & - & - & $1.08-2.13$ & - & - & - & - & - & - \\
\hline $\begin{array}{l}\text { Malvidin-O- } \\
\text { rhamnoside }\end{array}$ & - & - & - & - & - & - & $0.13-0.63$ & - & - & - & - & - & - \\
\hline Caffeoylisocitrate & - & - & - & - & - & - & 0.35 & - & - & - & - & - & - \\
\hline $\begin{array}{c}\text { Caffeic } \\
\text { acid-O-hexoside }\end{array}$ & - & - & - & - & - & - & $0.4-0.56$ & - & - & - & - & - & - \\
\hline Myricetin-O-hexoside & - & - & - & - & - & - & $0.19-0.29$ & - & - & - & - & 29 & - \\
\hline $\begin{array}{l}\text { Pelargonidin-3- } \\
\text { glucoside }\end{array}$ & - & - & - & - & - & - & - & $17.82-20.85$ & - & - & - & - & - \\
\hline $\begin{array}{l}\text { Pelargonidin-3- } \\
\text { malonylglucoside }\end{array}$ & - & - & - & - & - & - & - & $5.51-8.16$ & - & - & - & - & - \\
\hline $\begin{array}{l}\text { Pelargonidin-3- } \\
\text { glucoside }\end{array}$ & - & - & - & - & - & - & - & $114-348$ & - & 17.58 & - & - & - \\
\hline $\begin{array}{l}\text { Pelargonidin-3- } \\
\text { rutinoside }\end{array}$ & - & - & - & - & - & - & - & $18-62$ & - & - & - & - & \\
\hline
\end{tabular}

Note: Atmospheric-pressure chemical ionization, APCI; Diode array detector, DAD; dry extracts, DE; dry weight, dw; Electron spray ionization, ESI; fresh weight, fw; Hexahydroxydiphenoyl HHDP; High pressure liquid chromatography, HPLC; Liquid chromatography, LC; Lycium barbarum glycoprotein, LbGp; Lycium barbarum polysaccharides, LBP/LBPC/LBPA/LBPF; Mass spectrometry, MS; Nuclear magnetic resonance, NMR; reverse phase, RP; photodiode array detector, PDA; Quadrupole Time-of-Flight Mass Spectrometry, QTOF-MS; Ultra High pressure liquid chromatography, UPLC. * mg compound/mg extract. ${ }^{* *} \mathrm{mg} / 100 \mathrm{~g}$ of sample $\mathrm{dw}$. ${ }^{* * *} \mathrm{nmol} / \mathrm{g}$. 


\section{Bilberries}

Bilberries (BBs, Vaccinium myrtillus) are rich in quercetin, anthocyanins, tannins, catechins, vitamins, and pectins [252]. However, the most important classes of compounds considered responsible for the therapeutic role of $\mathrm{BB} / \mathrm{BB}$ extracts (BBEE) are phenolic acids and anthocyanins. The majority of compounds belonging to these two classes are presented in Table 2. The phenolics of blueberries varied widely and comprised of $0.3 \%$ of fresh fruits, which usually ranged from 48 to $304 \mathrm{mg} / 100 \mathrm{~g}$ of fresh fruit. Among the phenolic acids, the most abundant phenolic acids were ascorbic acid, chlorogenic acids, and 3-caffeoylquinic acid followed by caffeic, ferulic, ellagic, and gallic acids. Among the free phenolic acids, chlorogenic acids and ascorbic acids are of prime importance with reference to their health promoting activities [39]. Additionally, at least 15 different BB anthocyanins have been identified including the antidiabetic anthocyanin aglycones, which constituted $>70 \%$ of the total anthocyanin of BB (Table 2) [17,38,39]. BB anthocyanins showed excellent in vitro $\alpha$-amylase and $\alpha$-glucosidase inhibitory activities, reducing or preventing intestinal glucose absorption, and redirecting lipoprotein metabolism regulator enzymatic activities [43]. BB anthocyanins also inhibited advanced glycation end-product (AGE) formation, a severe diabetic complication. The main bioactive compounds considered responsible for inhibiting AGE activity were chlorogenic acid, quercetin-3-galactoside, quercetin-3-arabinoside, quercetin-3-glucoside, quercetin glycoside, quercetin-3-rhamnoside, myricetin glycoside 4, myricetin, and procyanidin b2 biomarkers [253]. BB polyphenols regulate hexose transport via GLUT2 and Na-glucose co-transporter 1 (SGLT-1), which assists glucose uptake. In other studies, GLUT2-mediated hexose transport was impeded by BB-derived flavones [48,254]. Cermak et al. [255] also reported that quercetin-3-O-glucoside and quercetin-4-O-glucoside decreased intestinal hexose absorption by inhibiting SGLUT1 in pig jejunum brush-border-membrane vesicles.

In one of the in vivo studies, supplementation with bilberry extract (BBE) reduced fasting blood sugars (FBS), total glyceraldehyde (TG), TC, and LDL-C levels. BB ingestion increased islet of Langerhans size and minimized retinopathy prognosis. BBE ingestion improved insulin sensitivity and hypoglycemia by upregulating AMPK, which upregulated GLUT4, PPAR- $\alpha$, ACOX, and carnitine palmitoyltransferase- 1 and ACPT-1A, which is synonymous to the suppression of glucose production and increased insulin sensitivity [15]. In another crossover study, the lyophilized BBE showed an $18 \%$ decrease in (incremental rise of) plasma glucose levels in overweight/obese diabetic humans, accompanied by decreased plasma insulin levels [48]. Recently, Alnajjar et al. [49] also reported that BBE anthocyanins reduced plasma glucose, oral glucose tolerance test (OGTT), TC, high-density lipoprotein cholesterol (HDL-C), LDL-C, TG, and inflammatory adipokine [leptin, TNF- $\alpha$, and high-sensitivity CRP (hs-CRP)] levels, without affecting the plasma Trolox equivalent antioxidant capacity (TEAC). The anti-inflammatory role of BB was also witnessed when BB juice (BBJ) consumption in healthy adults also reduced NF- $\mathrm{B}$-regulated inflammatory mediator expression (CRP, IL-6, IL-15, and monokine induced by gamma-interferon) and increased plasma levels of quercetin (by 32-51\%) and $p$-coumaric acid [51]. Later on, Kolehmainen et al. [53] examined the anti-inflammatory mechanism associated with $\mathrm{BB}$ consumption and reported the regulation of cytoplasmic ribosomal protein expression and the toll-like receptor (TLR) signaling and $\beta$-cell receptor signaling pathways, with decreased proinflammatory macrophage and monocyte functional gene expression including $C$ - $C$ chemokine receptor 2 and monocyte-to-macrophage differentiation. Kim et al. [127] also reported that daily BBE consumption reduced vascular permeability by reducing vascular endothelial growth factor levels in diabetic rats, in addition to restoring tight junction protein expression including claudin-5, zonula occludens-1, and occludin [127].

An accumulated number of evidence has also suggested that $\mathrm{BB}(\mathrm{E})$ intake is also helpful in relieving the oxidative stress and oxidative stress-related complications in obese and (pre)-diabetic subjects (Table 1). BBE administration alleviated stress-induced liver damage by decreasing plasma alanine aminotransferase (ALT), malondialdehyde (MDA), and nitric oxide (NO) levels and increasing glutathione (GSH) and vitamin C levels [45]. Capillary albumin filtration (CAF) is an early diabetic complication, associated with neuropathy and hypertension. BB anthocyanins 
prevented experimentally-induced-CAF, improving vision and retinopathy, and remarkable CAF reductions were observed among diabetic patients $[46,48,256]$. The suggested mechanism for inhibiting CAF involves BB anthocyanosides, which reduced aldose reductase activity and acted as strong antioxidants or pro-reductants, inhibiting AMP and guanosine monophosphate phosphodiesterase by scavenging superoxide anions [256]. Albumin retention (AR) was assessed by the isotopic CAF test in STZ-induced diabetic rats after anthocyanoside-rich BBE administration [46], and BBE treatment was found to reduce and maintain reduced AR (14\% to $1.3 \%)$ and low-frequency/high-frequency $(\mathrm{LF} / \mathrm{HF})$ ratio values in diabetic rats, without toxic effects [47]. BB-derived phenols increased the population of beneficial SCOA-producing gut bacteria (Lactobacillus spp. and Bifidobacterium spp.) and reduced bacterial metabolic syndrome biomarker genera including Enterobacteria. The dysbiosis symbolic Firmicutes/Bacteroidetes ratio, IR, and obesity-led-dysbiosis also decreased following BB consumption [49]. BB added to a fermented oatmeal drink caused a high glucose response, with a significantly reduced insulin index (Table 1) [50].

\section{Cranberries}

Cranberries (CrBs, Vaccinium macrocarpon) have also been intensively investigated for their proclaimed favorable cardiometabolic and dysmetabolic syndrome effects, likely due to phytochemicals such as oligosaccharides, procyanidins, and anthocyanins. A comprehensive list of potential well-known antioxidative, antidiabetic, and anti-inflammatory compounds found in $\mathrm{CrB}$ (products) or $\mathrm{CrB}$ extracts $(\mathrm{CrBE})$ used in clinical or non-clinical interventional studies are listed in Table 2 [226-228]. The purified fractions of procyanidins were more antidiabetic potent than the anthocyanin and oligosaccharide fractions [257]. With respect to individual compounds, quercetin-3-galactoside, 5-caffeoylquinic acid, and quercetin-3-rhamnoside were the major compounds comprising $75-77 \%$ of total flavonols of cranberry whilst 4-caffeoylquinic acid, 3-caffeoylquinic acid, quercetin-3-arabinopyranoside, myricetin3-galactoside, quercetin, quercetin-3-arabinofuranoside, and quercetin-3-benzoylgalactoside were found in the least amounts. Many authors have initially described the in vitro antidiabetic/antiglycation activities of cranberry extracts or its products [226,257]. Barrett et al. [227] isolated ellagitannins and proanthocyanidins and demonstrated their dose-dependent inhibition of $\alpha$-amylase and glucoamylase activities. CrB powder from stress-adapted portions of cranberry juice (CrB-JSB) showed increased $\alpha$-amylase and glucoamylase activities compared with CrB powder, and CrB-JSB $(200 \mathrm{mg} / \mathrm{mL})$ also showed anti-hypertensive properties by inhibiting the angiotensin I-converting enzyme (ACE-1) activity [228]. Podsedek et al. [258] found that CrB extracts inhibited pancreatic lipase activities more potently than other berries, but digestive enzyme inhibitory activities were less potent. Purified $\mathrm{CrB}$ proanthocyanidins and oligosaccharides also reduced the levels of $\mathrm{HbAC} 1$ levels from $7.05 \%$ to $5.75,5.55$, and $5.45 \%$ in the hemoglobin-glucose assay, whereas the recommended $\mathrm{HbAC} 1$ value should be below $7 \%$, according to the American Diabetes Association. Reduced glucose-induced AGE formation during middle glycation stages was also observed during the human serum albumin (HSA)-methylglyoxal and HSA-glucose assays [257]. CrB-derived phenolic-rich extracts decreased fluorescent AGE generation by almost $60 \%$, which was more effective than the other berry anti-AGE activities of raspberries, apples, grapes, and strawberries. The $\mathrm{CrB}$ anthocyanin and procyanidin fractions also decreased fluorescent AGE generation in an arginine-methylglyoxal model by 53.3 to $56.8 \%$ [226]. The $\mathrm{CrB}$ oligosaccharide-rich fraction showed concentration-dependent anti-glycation activity, which reduced AGE formations by 53.3 to $56.8 \%$, respectively, almost as strongly as the reference compound [259].

The hypoglycemic, hypo-insulinemic, and hypolipidemic properties of $\mathrm{CrB}$ or its byproducts have also been reported in many clinical interventions (Table 1) [5,56-60,260]. Low-calorie dried cranberry (LCDC, $40 \mathrm{~g}$ ) consumption after HFD reduced hyperglycemic and hyperlipidemic conditions, halted increases in IR/HOMA-IR and inflammatory biomarkers (TNF- $\alpha$ IL-6, IL-2, IL-10, IL-18, malondialdehyde-MDA) in adipose tissue, and lowered plasma lipid oxidation and oxidative stress biomarker levels in the treated group [56]. After testing LCDC, sweetened, dried CrBs (SWDC) 
consumed by non-insulinemic diabetic patients also reduced plasma glucose levels when compared with white bread (WB) and unsweetened dried CrBs (USCB) [260]. The plasma insulin peak following SWDC consumption appeared earlier than the insulin peaks for WB or USCB consumption and was significantly lower than those for WB and USCB. Bread consumption induced higher insulin and postprandial glucose responses, which could be diminished by incorporating CrBs [58-60,260]. CrB extracts (CrBEs) also halted visceral adiposity and weight gain in HFD-fed C57BL/6J mice, and improved HFD-induced hypercholesterolemia, hypertriglyceridemia, antioxidant defense mechanisms, and hepatic oxidative stress and normalized the NF- $\mathrm{B} / \mathrm{I} \kappa \mathrm{B}$ ratio [54]. Long-term $\mathrm{CrBE}$ consumption effects were also investigated [55,63], and the addition of CrBE to normal chow delayed age-related basal plasma insulin concentration declines [63]. CrBE supplementation also improved glucose responsiveness and increased insulin concentrations (7.6\%) in rats, without significant HOMA-IR changes. CrBEs also induced duodenal homeobox 1 and insulin expression within islets, which enhanced insulin release, suggesting insulinotropic effect of cranberry intervention [55]. CrBEs showed the anti-obesity effect by inducing the LDL receptor expression, resulting in increased hepatic cholesterol uptake and promoted cholesterol binding to bile acids, causing increased fecal cholesterol excretion [57]

$\mathrm{CrBJ}$ consumption was also examined in randomized clinical studies (Table 1). Healthy adults who consumed $\mathrm{CrB}$ juice (CrBJ) also showed reduced proinflammatory CRP levels [61]. Daily CrBJ supplementation for 60 days increased paraoxonase-1 (PON-1) and apolipoprotein (Apo)A-I expression (dysfunctioning of PON-1 and apoA-I results in glycation in T2DM patients) accompanied by decreased blood glucose and ApoB levels in T2DM patients. CrBJ inhibited GLUT-4-mediated gastric glucose uptake and aldose reductase, $\alpha$-amylase, and $\alpha$-glucosidase activities and protected LDL-C against oxidation [60,64,228]. Moreover, both routine-calorie CrBJ (RCCJ) and high-calorie $\mathrm{CrBJ}$ (HCCJ) are enriched in hexoses and sugars, which could limit their use by diabetic individuals. Therefore, low-calorie CrBJ (LCCJ) was examined in glycemic and insulinemic T2DM patients by Wilson et al. [58,59] and Novotny et al. [65]. LCCJ consumption did not affect LDL-C, HDL-C, or TC levels; however, ApoA-I, ApoA-II, ApoB, and TG levels were reduced in the treated group. Individuals with higher baseline TG or HOMA-IR values experienced more pronounced drops in TG and HOMA-IR than others [65]. Serum HbA1c levels were reduced by $11.4 \%$ and $6.02 \%$ following RCCJ and RCCJ enriched with omega-3 fatty acid consumption. Omega-3 fatty acid-enriched RCCJ also increased HDL-C levels by $21.1 \%$ compared with the baseline [63]. Additionally, folic acid consumption combined with LCCJ decreased plasma homocysteine levels and increased adiponectin and folic acid levels without any change in inflammatory biomarker levels (IL-6, IL-10, IL-18, and TNF- $\alpha$ ) [66].

In summary, $\mathrm{CrB}$ consumption exerted antimetabolic syndromic effects by downregulating GLUT2 and GLUT4 expression and increasing hepatic cholesterol uptake. Diet-induced weight gain and low-grade inflammation were counteracted by the prevention of TG accumulation and strengthened antioxidative defense mechanisms. The other proposed possible mechanisms of action of $\mathrm{CrB}$, or its products, consumption include reduction and inhibition of ACE-I activity and oxidative stress, accompanied by improvements in endothelium-dependent vasodilation. Furthermore, CrB-derived bioactive compounds including quercetin, inhibited microsomal TG transfer protein (MTP), preventing ApoB-containing lipoprotein assembly. Quercetin also lowered proinflammatory CRP expression in a transgenic mouse model and decreased cytokine-induced CRP expression in Hep3 $\beta$ cells and Chang liver cells $[68,69,261]$, which was analogous to weight lossand polyunsaturated fatty acid (PUFA)-rich Mediterranean diet-induced CRP suppression [65,261]. Additionally, $\mathrm{CrB}$ consumption has beneficial effects on the gut microbiome. HFD reduced Bacteroidetes and increased Firmicutes populations in $\mathrm{C} 57 \mathrm{Bl} / 6 \mathrm{~J}$ mice, which was reversed by CrBEs intake. CrBEs also increased the Akkermansia gut population, which may prevent HFD-induced increases in circulating pro-inflammatory lipopolysaccharides (LPS) [54]. 


\section{Raspberries}

Raspberries (RBs), especially red RBs (Rubus idaeus L.), are rich in fiber and potent therapeutic phytochemicals that have rendered raspberries as a functional food for metabolic syndrome [199]. The phytochemicals of raspberries provide the healthy and protective affects to its consumers by influencing the cell signaling pathways that affect transporters, receptors, cellular events, and gene expression. These health promoting RB phytochemicals belong to ellagitannins and anthocyanins (Table 2) [262]. Among these two classes, RB anthocyanins are major contributors to health promoting bioactivities. The anthocyanins of RB are cyanidin-based, but with dissimilar glycosidic units. The pelargonidin-based anthocyanins are only found in RB and strawberries with a sophoroside unit attachment unique to raspberries. Ellagitannins are hydrolyzable tannins that represent another major RB phytochemical group, which are hexahydroxydiphenoyl esters with quinic acid or glucose cores. Glucose cores can attach to galloyl groups, and further arrangements within hexahydroxydiphenoyl molecules yield the ellagic acids. Numerous in vitro studies have described that RB extracts (RBE) reduced lipid oxidation, LDL-oxidation, ROS generation, and DNA damage, associated with upregulated CAT and SOD enzymatic antioxidant activities [73]. Hypoglycemic studies revealed that RBEs inhibited $\alpha$-amylase, with mixed effects on $\alpha$-glucosidase, and aglycones and anthocyanin promoted GSIS from pancreatic cells $[263,264]$

Fresh RB extracts (RBEs) and freeze-dried RB powder have also been employed for in vivo evaluation (Table 1), in which oxidative stress was found to be relieved as decreased protein and lipid oxidation and damage was seen $[74,75]$. RB freeze-dried powder fed to obese and diabetic mice reduced ROS levels in erythrocytes by $0.87 \%$ when compared to the controls, indicating the ROS-neutralizing role of RB powder bioactive constituents during homeostasis. The RB intervention reduced ROS levels by increasing the glutathione peroxidase (GPx)/SOD ratio (2\%) and GPx activity $(2.13 \%)$ when compared to the placebo controls. Upregulated GPx activity also inhibited lipid peroxidation and protected against diabetes by delaying perturbed metabolism development [76]. RB juice (RBJ) given to hypercholesterolemic golden Syrian hamsters reduced plasma LDL-C levels and increased hepatic GSHPx and SOD activities by $30 \%$ and $25 \%$, respectively [72]. Polyphenol-rich black RBs have also been combined with HCD foods for sustainable postprandial glycemic control, reducing plasma free fatty acid (FFA) and oxidative stress marker levels. RBs, combined with HCD, blunted postprandial insulinemia and ex vivo LDL-oxidation during the postprandial state, hindering glucose uptake (Table 1) [91]. Purified hydrolyzable RB tannin supplementation in rat gastritis models also demonstrated increased endogenous antioxidant defense system components and decreased inflammatory biomarkers and conditions. RB ellagic acid suppressed the specific immunoglobulin antibody response in cytotoxic cells without affecting other immunoglobulin parameters. Reduced lipid peroxidation, neutrophil infiltration, and iNOS overexpression were observed in ex vivo gastritis and Crohn's disease models $[85,86]$. A recent study showed that RBE consumption mitigated carcinogenic acrylamide-induced liver toxicity in male Wistar rats. RB treatment increased plasma antioxidants enzyme levels and reduced acrylamide-induced hepatic ALT, aspartate aminotransferase (AST), alkaline phosphatase (ALP), lactate dehydrogenase (LDH), and gamma-glutamyltransferase ( $\gamma$-GT) activities [265].

Limited human clinical trials have been performed with RBs, but the antidiabetic effects of RBEs and purified compounds have been examined in diabetic rat models (Table 1$)[87,88]$. Numerous anthocyanin and polyphenolic components have been hypothesized to affect starch digestion, altering postprandial glucose levels [263]. RB anthocyanin also enhanced insulin sensitivity, upregulated adiponectin expression, downregulated inflammatory cytokines, and altered AMPK phosphorylation, which is a T2DM therapeutic target [264]. A clinical trial examined RB intake with a HCD and reported no postprandial insulin and glucose response alterations [92,93]. In another study following HC-bar consumption, RB intake increased postprandial glucose levels, without changing peak glucose concentrations, and diminished postprandial insulinemia [91]. RB effects on IR and the underlying mechanisms in skeletal muscles were studied by Zhao et al. [87]. AMPK inactivation led to skin lipid 
accumulation and insulin sensitivity loss. This study found that AMPK- $\alpha 1$ is important for AMPK activation, and dietary RB powder inclusion increased insulin sensitivity by upregulating cytochrome $C$ protein in AMPK- $\alpha 1^{+/+}$rats [87]. The supplementation of $5 \% \mathrm{RB}$ with HFD improved insulin sensitivity by increasing IRS-1 phosphorylation at Tyr 612 and increasing the p-Akt/Akt ratio. RB intake also attenuated nod-like receptor pyrin containing 3 (NLRP3) inflammasome activation, which is a major contributor to metabolic syndrome. NLPR3 activation, combined with caspase 1, forms caspase 1p20 and caspase 1 p10. Caspase 1 p20 activation releases IL-1 $\beta$ and IL-18. RB consumption downregulated NLPR3, caspase 1p20, IL-1 $\beta$, and IL-18 expression in HFD-fed mice [88]. Recently, Zou et al. [266] also reported that $5 \%$ RB powder supplementation with HFD suppressed TNF- $\alpha$, L- 6 , IL-1 $\beta$, and NF- $\kappa$ B p65 expression and increased GLUT4 expression and IRS-1 and Akt phosphorylation. RB powder also increased mitochondrial biogenesis genes (PGC-1 $\alpha$ and Nrf1) and mitochondrial abundance markers (cytochrome c, citrate synthase, and cytochrome c oxidase subunit IV) [266].

The health-promoting effect of raspberry supplementation on the glycerophospholipids metabolism is also evident (Table 1). The addition of $10 \%$ freeze-dried RB to an isocaloric diet increased plasma HDL-C (1.5\%) and insulin sensitivity and decreased abdominal fat (38\%), blood TG, cholesterol, ROS (19\%), and LDL-C (0.3\%). Similarly, RB-derived cyanidin-3-glucoside upregulated GLUT4 expression, without affecting insulin sensitizer adiponectin [89]. Ellagic acid, which is unique to RB, increased insulin secretion and decreased FBS, HbA1c, and glycated urinary albumin levels. RB inclusion in HFD/HCD diminished impaired insulin tolerance and inflammatory cytokines. RB seed flour, combined with a HCD, downregulated the lipogenic gene expression of lipoprotein lipase (LPL), stearoyl CoA desaturase-1 (SCD-1), and diacylglycerol acyltransferases 2 (DGAT2) and gluconeogenesis promoting genes including PEPCK, G6Pase, sterol regulatory element-binding protein 1c (SREBP-1c), and carbohydrate response element-binding protein (ChREBP) (Table 1) [90]. RB ketones also prevented HFD/HCD-induced BW gains, reduced visceral and adipose tissue, reduced hepatic TG contents, and increased norepinephrine-induced lipolysis in white adipocytes, suppressing lipid accumulation by enhancing lipolysis and fatty acid oxidation [267]. RB supplementation in diabetic patients substantially lowered postprandial glucose levels, without affecting plasma insulin levels after a fatty meal challenge [94]. RB consumption also reduced TG levels [71]. Conflicting results regarding $\mathrm{RB}$ interventions and effects on metabolic syndrome biomarkers have been reported. Noratto et al. [76] found an insignificant difference in the weight gain between diabetic mice fed with and without RBs. Similarly, Kirakosyan et al. [77] and Norrato et al. [76] reported no RB intervention effects on LDL-C, fasting blood insulin, IKB $\alpha$, and PPAR- $\gamma$ levels. Contrasting results may be due to higher baseline weights of the subjects. However, Kirakosyan et al. showed that RB intake reduced glucose metabolisms and insulin signaling mRNA levels including MAP2K1, glycogen synthase (GYS1), hexokinase, I $\mathrm{B} \beta$, phosphatidylinositol-4,5-bisphosphate 3-kinase, mechanistic target of rapamycin (mTOR), Chuk (involved in innate immunity), C-X-C chemokine receptor type 4 (involved in inflammation), LPL, GYS1, MAP2K1(involved in apoptosis), nicotinamide phosphoribosyltransferase, ApoE, PPAR- $\gamma$, and PPAR- $\alpha$ (involved in glucose and lipid dynamics) (Table 1) [77].

RB intake also increased gut Lactobacillus, which is a healthy gut marker, and increased beneficial gut intestinal SCFAs, which are colonic epithelial cell substrates and improve gut health [78]. RB consumption increased SCFA-producing bacterial populations including Bacteroides, Butyricimonas, Ruminococcus, Akkermansia, Clostridium butyricum, Mucispirillum, Oscillibacter, Ruminococcaceae, and Lachnospiraceae, which improved metabolic syndromic conditions during metformin T2DM treatment [268]. Furthermore, RB consumption time- and dose-dependently increased the gut microbial population of Anaerostipes, Ruminococcus, Akkermansia, Coprobacillus, Allobaculum, Anaerovorax, Dorea, Asaccharobacter, Anaerotruncus, Coprobacillus, Desulfovibrio, Victivallis, and Mucispirilum, and decreased the microbial population of Acetivibrio, Anaerotruncus, Bifidobacterium, Lactococcus, Prabacteroides, Streptococcus, Turicibacter, and Acetivibrio. Increased beneficial microbial communities as above-mentioned can reduce inflammation, obesity, metabolic syndrome, and dysbiosis [79]. $\mathrm{Su}$ et al. [80] reported that RB-derived pelargonidin-3-O-glucoside increased the gut population of 
Prevotella and improved the Bacteroidetes/Firmicutes ratio. Another more recent report concluded that there was a favorable higher population of Akkermansia muciniphila and Bacteroidetes/Firmicutes ratios in pathogenic free mice fed on black RB powder [81]. Conclusively, RB consumption showed antidiabetic effects, inhibiting glucosidase and amylase activities, strengthening the endogenous antioxidant defense system, reducing inflammatory biomarkers, activating AMPK, GLUT2/GLUT4, IRS-1 phosphorylation, downregulating lipogenesis and gluconeogenesis genes, and increasing epithelial mucus barrier protecting and SCOA-producing bacterial populations (Table 1) [83,84].

\section{Mulberries}

Mulberries (MBs, Morus alba/Morus rubra) are rich in cyanidin-3-glucoside, cyanidin-3-rutinoside, and pelargonidin-3-glucoside, and other anthocyanins comprising $78 \%$ of the MB polyphenolic compounds (Table 2) [269]. These purified anthocyanins from MB showed excellent glucose-lowering properties in HepG2 cells, increasing PPAR- $\alpha$ and AMPK phosphorylation (activation) and the $\mathrm{p}$-mTOR/mTOR ratio (synonymous with the activation of insulin receptors and insulin-like growth factor 1 receptors). During metabolic syndrome, IRS-1 inactivation increases the p-p38/p38 ratio (subfamily of MAPK, which requires inflammatory cytokines for activation) and reduces PGC- $1 \alpha$ expression (a regulator of energy homeostasis and mitochondrial biogenesis), which were abolished or reversed with MB anthocyanins treatment [269]. In addition to anthocyanins, polyphenol-rich MB methanolic extracts also showed excellent $\alpha$-glucosidase inhibitory activities due to quercetin 3-O-rutinoside, chlorogenic acid, and cyanidin 3-O-glucoside [235]. Cyanidin glycosides in MBs also reportedly possess potent anti- $\alpha$-glucosidase activity, which inhibit the enzyme by affecting $\alpha$-glucosidase $\alpha$-helix contents via cyanidin-3-glucoside (C3G) and cyanidin-3-rutinoside (C3R) domain matching [270]. HepG2 cells treated with the five most abundant MB polyphenols including C3G, 1-deoxynojirimycin, resveratrol, C3R, and oxyresveratrol showed improved glucose consumption and postprandial glucose disposal through increased glucokinase activity [271]. Another study found that 1,5-dicaffeoylquinic and dihydroquercetin acid protected cells against glucotoxicity [29]. MB extracts (MBEs) upregulated PGC-1 $\alpha(38 \%)$ and FOXO1 (40\%) (regulator of PEPCK and G6Pase enzymes) and downregulated PEPCK (79\%) and G6Pase (37\%) expression in IR model cells. MBEs also upregulated AKT2 (crucial for IRS activation and hence increasing insulin sensitivity) and glycogen synthase kinase (GSK) $3 \beta$ levels, with significantly increased $p$-AKT/AKT ratios (hence reduced IR) and increased GSK3 $\beta$ phosphorylation and glycogen synthase 2 (GSY2) activation [272].

In in vivo studies, MB polyphenols and polysaccharides reduced ROS levels and enhanced reductant enzymatic activities including GPx, SOD, and CAT while reducing IL- 8 , TNF- $\alpha$, COX-2, and IL-6 release in STZ-induced diabetic mice (Table 1) [273]. MB anthocyanins also attenuated HFD-induced decreased hepatic SOD and GPx activities [95]. Yan et al. [269] reported that MB anthocyanins alleviated hypoglycemia by inhibiting ROS generation, promoting AMPK phosphorylation, activating tuberous sclerosis 2, (reducing the mTOR and ACC signaling), reducing p38-MAPK and PGC-1 $\alpha$ expression, and increasing mitochondria and matrix metalloprotease (MMP) abundance in diabetic mice (Table 1) [97]. MB wine consumption by diabetic mice also reversed glycemic status, with reduced oxidative stress markers, proteinuria, non-esterified fatty acid contents, and lipid peroxidation and improved antioxidant defense systems [97]. MB-derived and purified cyanidin-3-O- $\beta$-D-glucopyranoside intervention also circumvented diabetic cytopathy by reducing oxidative stress markers of DNA modification including 8-hydroxy-2-deoxyguanosine and increasing the axonal transport of nerve growth factor [98].

The oral MBE supplementation also improved insulin signaling by decreased GSK3 $\beta$, and increased GSY2, AKT, increasing p-AKT/AKT ratios in skeletal, hepatic, and adipocytes tissues of diabetic mice [272]. Oral MB fruit intake in diabetic mice also improved insulin sensitivity by upregulating (up to $3 \%$ ) the IRS-1, p-IRS01/IRS-1, p-AMPK/AMPK, CCAAT-enhancer-binding proteins (C/EBP), sterol regulatory element-binding protein 1 (SREBP-1c), and PGC-1 $\alpha$ [269,274]. Ren et al. [99] further reported that MB consumption normalized glucose metabolism by 
abolishing protein-tyrosine phosphatase $1 \mathrm{~B}$ expression and activating the phosphoinositide-3-kinase (PI3K)/AKT pathway. MB anthocyanin-induced p38-AMPK-PGC-1 $\alpha$ pathway upregulation increased thermogenesis gene activity. Anthocyanin components also downregulated lipogenesis genes including hydroxymethylglutaryl coenzyme A reductase (HMG-CA-R), SREBP-1c, and FAS [100] and activated scavenger receptor class B type 1 and ATP-binding cassette transporter (ABCA1), which transfer cholesterol.

MBEs combined with HFD demonstrated excellent anti-obesity and hypolipidemic properties. MBE supplementation reduced BW gains by $41.3 \%$ in HFD-fed diabetic male C57BL/6 mice. Serum TG, TC, HDL-C, and LDL-C levels in HFD + MBE-fed mice were lower than those in HFD-fed diabetic mice, but higher than the MBE-fed controls. Liver injury parameters (ALT and AST) were reduced in HFD + MBE-fed mice, with reduced adipose and hepatic liver lipid droplet sizes [101]. MB fruit consumption lowered TG, TC, LDL-C, and FFA levels in other studies (Table 1) [102,103]. MB-derived anthocyanin consumption decreased serum levels of inflammatory markers (IL-6, IL- $1 \alpha$, iNOS, TNF- $\alpha$, IFN- $\gamma$, and NF- $k B$ ), thiobarbituric-acid-reactive substances (TBARS) (a lipid oxidation marker), hyperlipidemic markers (TC, glucose, TG, and leptin), insulin, and hepatic AST, ALP, and ALT levels, downregulated FAS, and increased heme oxygenase-1 (HO-1) (a cytoprotective enzyme) and antioxidant enzyme levels in HFD-fed male C57BL/6 mice (Table 1) [95,104]. Aqueous MBEs employed the hypolipidemic and hypoglycemic effects by activating the AMPK, increasing the p-AMPK/AMPK ratio (hence improving mitochondrial biogenesis), and downregulated FAS, acetyl coenzyme A carboxylase (ACC), glycerol-3-phosphate acyltransferase (GPAT), and SREBP-1 [104]. MBEs in HFD-fed male Sprague-Dawley rats prevented non-alcoholic fatty liver disease (NAFLD) by downregulating lipid/cholesterol homeostasis-related genes (FAS, ACC, GPAT, and SREBP-1) and suppressing the lipid oxidation biomarkers MDA and 4-hydroxynonenal [105,106]. Hu et al. [275] demonstrated that MBE increased nuclear factor erythroid-2-related factor 2 (Nrf2) phosphorylation and nuclear translocation, activating the Nrf2/antioxidant response element signaling pathway, which increased quinone oxidoreductase $1, \mathrm{HO}-1$, and $\mathrm{NAD}(\mathrm{P}) \mathrm{H}$ expression and promoted antioxidant enzymatic activities, thus protecting hepatocytes against palmitic acid-induced lipo-toxicity and oxidative stress.

Gut microbiota regulates dietary energy harvesting, glucose homeostasis, and lipid metabolism, especially in brown adipose tissues (BAdT). Mitochondria-rich BAdT activation can increase energy expenditure following MB-induced UCP1 upregulation and oxidative phosphorylation downregulation, releasing energy as heat. $\mathrm{MB}$ powder consumption reversed HFD-induced gut microbiome changes, increasing the Bacteroidetes/Firmicutes ratio and Bacteroidetes populations (Porphyromonadaceae, Parabacteroide, S24-7, Prevotellaceae, Alloprevotella, Rikenellaceae, Alistipes, Rikenella) and decreasing the Proteobacteria (Alphaproteobacteria, Brevundimonas, Devosia, Rhodobacteraceae, Polymorphobacter, Deltaproteobacteria, Desulfovibrio, Arenimonas), and Firmicutes (Clostridia, Lachnospiraceae, Eubacterium, Coprococcus, Ruminococcaceae, Oscillibacter, Ruminiclostridium) populations $[107,108]$. At the genus-level, MB fruit supplementation promoted SCOA/SCFA-producing and IMBD-restoration-supportive genera Lactobacillus, Bacteroidales, Bacteroides, Allobaculum, and Akkermansia growth, and suppressed Corynebacterium, Staphylococcus, Aerococcus, Jeotgalicoccus, Facklamia, and Enterococcus growth. Allobaculum and Lactobacillus protect against metabolic syndrome, and both genera increased in diabetic rats after MB intake [108]. Approximately 60 metabolites were identified in MB including flavonols, phenolic acids, flavonoids, lignans, and organic acids (Table 2) [234]. In short, MB fruit consumption upregulated/activated glucose-consumption-related pathways and insulin-sensitivity-related pathways (p-AKT/AKT ratio, glucokinase, PGC-1 $\alpha$, FOXO1, IRS-1, p-IRS-1/IRS-1, p-AMPK/AMPK, C/EBP, and Bacteroidetes/Firmicutes ratio) and downregulated lipogenesis-related pathways (FAS, ACC, GPAT, and SREBP-1) in skeletal, hepatic, and adipocyte tissues.

\section{Lingonberries}

Lingonberry (LB, Vaccinium vitis-idaea) alleviates metabolic syndrome including frequent urination and fatigue. In in vitro studies, LB extracts (LBEs) increased glucose uptake in C2C12 skeletal 
muscle cells by modulating AMPK activity [276]. LB polysaccharides inhibited $\alpha$-glucosidase activity (by 118-136\%) more strongly than the referenced acarbose [277]. In in vitro digestibility assays, LB polyphenols $(7 \% w / v)$ were added to white rice, which significantly reduced glucose release [278]. Ethanolic LBEs demonstrated antiglycation activity, with AGE inhibition majorly mediated by LB cyanidin-3-galactoside, quercetin-3-galactoside, and (+)-catechin [279]. In J774 macrophages, LBEs significantly inhibited LPS-modulated NO production, without substantial effects on COX-2 or iNOS expression. Proinflammatory cytokine (IL-6, IL-1 $\beta$, and TNF- $\alpha$ ) expression was reduced by TNF- $\alpha$ downregulation, IKB receptor degradation inhibition, and reduced extracellular signal-related kinase 1/2 phosphorylation [280]. However, in RAW 264.7 macrophages and activated 3T3-L1 adipocytes, LBEs mitigated oxidative stress by suppressing COX-2, iNOS, TNF- $\alpha \alpha$, IL-6, MCP-1, and IL-1 $\beta$ expression [281].

In in vivo studies, LB consumption also improved hyperinsulinemic, hyperglycemic, and dyslipidemic conditions (Table 1) [111]. LBE consumption reduced blood glucose levels (17-25\%), obesity-induced hepatic steatosis (50-60\%), and plasma TG, TC, and LDL-C levels (12-18\%) associated with increased GLUT4 expression and AMPK and Akt phosphorylation, increasing glucose metabolism and hepatic fatty acid oxidation [111]. LB juice (LBJ) improved low-grade inflammation and endothelial function by increasing $\mathrm{NO}$ availability, which is necessary for the inhibition of adhesion molecules, MCP-1, ACE-1, COX-2, and other pro-inflammatory markers [112]. The LB-rich Okinawan-based Nordic diet improved anthropometric (BW, body mass index (BMI), and waist circumference) and metabolic (HOMA-IR, IR, FBS, TG, CRP, TC, and HDL-C) parameters [119]. Linderborg et al. [120] demonstrated that $\mathrm{LB}$ powder consumption compensated for additional glucose and lipid consumption. LBJ intake prevented HFD-induced BW gains in C57BL/6JBomTac mice. LB supplementation reduced FBS, fasting insulin, and HOMA-IR levels (Table 1) [113,114]. Hepatic lipid accumulation and liver function parameters (ALT, TG, and cholesterol) decreased after LB supplementation, more strongly than other berries $[113,114]$. In a recent hyperlipidic and hypercaloric meals challenge study, the LB supplementation halted increased cholesterolemia and decreased the glycemic response, $\mathrm{CRP}$, and postprandial endotoxemia [121]. In an atherosclerosis $\mathrm{ApoE}^{-/-}$mouse model, whole LB consumption upregulated bile acid synthesis gene Cyp7a1, increased the cecal propionic-acid-producing bacteria proportions, and decreased triglyceridemia and atherosclerosis [115]. The insulinemic and glycemic response following oat bread consumption was also checked. The LB polysaccharide and fiber consumption, following bread consumption, reduced glucose and CRP responses [122]. Whole LB and LB nectar intake reduced postprandial glucose and insulin levels after $35 \mathrm{~g}$ sucrose intake, and insulin levels increased more rapidly following LB than after glucose intake. Postprandial glucose levels were also reduced following LBJ consumption. Insulin and FFA changes after LBJ consumption were similar to those observed after whole fruit consumption (Table 1) [113,114,123].

Urinary metabolomics revealed that a LBJ-containing diet increased 4-hydroxyhippuric acid and hippuric acid excretion, whereas 4-deoxythreonic acid, 3-hydroxybutanoic acid, dimethylamine, creatinine, and citric acid excretion reduced, likely due to high polyphenolic compound and benzoic acid contents in LBJ (Table 2) [124,282]. Plasma lipidomics data showed that LB consumption increased health-promoting lyso-phosphatidylethanolamines, (LPE) (16:0), lysophosphatidylcholine (LPC) (20:5), (16:1), and (22:5), and phosphatidylcholines (PC) (33:2), (32:2), (35:6), (34:4), (36:6), and (36:5), whereas obesity and diabetes symbolic sphingomyelins (SM) (34:1), (33:1), (40:3), and (38:2) were reduced. Quinate levels also increased, and plasma alanine and glucose levels decreased significantly [116]. LBE and powder supplementation of HFD downregulated the expression levels of macrophage marker endothelial growth factor-like module containing mucin-like, hormone receptor-like 1 (EMR1), and LPS-sensing TLR4 (member of the toll-like receptor family activation of which results in signaling the NF- $\mathrm{kB}$ pathway and inflammatory cytokine production) and upregulated tight junction-associated occluding (an integral membrane protein whose modulation is associated with cellular proliferation, differentiation, signal transduction, and migration) and proglucagon (a precursor of glucagon from $\alpha$-pancreatic cells). The HFD-fed control microbiome showed the upregulation of the ATP-binding 
cassette $(\mathrm{ABC})$ transporter, cell motility, membrane transporter, bacterial chemotaxis, bacterial motility, the two-component system, flagellar assembly, transcription, and signal transduction genes, compared with the LB-treated group [283]. LB consumption enriched genes associated with lipid metabolism, nutrient transport, energy, nucleotides, and amino acids (Table 1) [113,114,117]. At the phyla level, LB supplementation affected the diversity and population of Firmicutes, Bacteroidetes, Proteobacteria, and Verrucomicrobia. The relative abundance of Bacteroidetes increased, and the relative abundance of Firmicutes decreased significantly, reducing the obesity and diabetes symbolic Firmicutes/Bacteroidetes ratio [113,114,117]. At the genus level, HFD increased Firmicutes genera populations including Lachnospiraceae, Oscillospira, and Ruminococcus. The abundance of Bacteroidetes increased following LB supplementation, due to unknown members of the S24-7 family. LB supplementation increased Parabacteriodes, Odoribacter, and Akkermansia populations. The principal component analysis confirmed LB extract-induced gut microbial profile variations. HFD increased the population density of the genera Oscillospira and Ruminococcus and the Lachnospiraceae family, microbes associated with diabetes pathogenesis progression [284], which was prevented by LB fruit/powder/extract consumption [285]. Akkermansia population increases were associated with the abundance of Akkermansia muciniphila species, which are known beneficial gut microbacteria that counteract HFD-induced adipose tissue inflammation, endotoxemia, BW gain, and IR in C57BL/6 mice [286]. Liquid chromatography (LC)-tandem mass spectrometry (MS/MS)-based LB fingerprinting identified several bioactive compounds responsible for antioxidative, antidiabetic, and anti-inflammatory properties. These bioactive compounds primarily belong to anthocyanidins, flavonols, glycosides, catechins, and different conjugates of ferulic and caffeoyl acid (Table 2). Depending on aglycon weight, cyaniding-containing compounds were the major bioactive compounds followed by proanthocyanidins, which represent phenolic compounds in LB $[236,237]$.

\section{Blackberries}

Blackberries (Rubus grandifolius L and Rubus fruticosus L.) are consumed fresh or as juices, jams, and liquors. Blackberries are enriched in health-promoting compounds (Table 2) belonging to flavanals, flavanones, flavonols (kaempferol and quercetin glycosides), anthocyanins, hydroxycinnamic acids, and caffeic acid conjugates. The high-performance liquid chromatography (HPLC)-electrospray ionization (ESI)-mass spectrometry (MS)-based Rubus grandifolius L. metabolic profiling revealed 50 phytochemicals including anthocyanins, hydroxycinnamic acids, flavonols, flavanones, and ellagitannins (Table 2) [131,238,239]. These blackberry-derived compounds offered an antidiabetic and anti-obesity role by inhibiting digestive enzymes ( $\alpha$ - and $\beta$-glucosidase, aldose reductase, lipase, and $\alpha$-amylase) and exhibiting anti-glycation abilities. The blackberry $\alpha$-glucosidase and $\alpha$-amylase inhibitory activity was superior to the reference compounds, Acarbose and 1-Deoxynojirimycin (1-DNJ) [287]. Anthocyanins are considered to be the primary mediator of blackberry extract anti-digestive activities, and glycosides are the primary inhibitors of $\alpha$-glucosidase activity. The interaction between glycosides and enzymes is considered to be competitive, suggesting that glycosides bind to enzymatic active sites [288]. Cytidine glycosides from leaf and fruit R. grandifolius extracts reduced aldose reductase activity, which is responsible for AGE accumulation in diabetic patients via dicarbonyl activity [289]. The recorded anti-glycation activity of BB fruit extracts was $\mathrm{IC}_{50}=1.87 \mathrm{mg} / \mathrm{mL}$, and ellagitannins and flavonols were the most prominent anti-glycation agents [131,238,239]. HepG2 cells incubated with gut microbial-fermented blackberry metabolites (GMBB) and gastrointestinal-digested BB slurry (GIDBB) showed improved glucose uptake. Increased HepG2 uptake also increased glycogen synthesis. GIDBB and GMBB also maintained the desired cellular redox status by neutralizing ROS and restoring the mitochondrial membrane potential. GIDBB and GMBB supplementation restored glutathione levels, strengthening the oxidative defense system [290].

In in vivo studies, blackberry-derived purified anthocyanin-enriched and ellagitannin-enriched fractions decreased lipid peroxidation markers (TBARS and MDA) and increased hepatic and 
brain antioxidant enzyme activities (CAT, GSH, SOD, and GPx) [125]. Similarly, blackberry extract consumption attenuated the HFD-induced effects in an obesity-prone mouse model and prevented the increase in metabolic and lipidemic parameters, while reinforcing endogenous and exogenous antioxidant enzyme systems (Table 1) [126]. LPL activity, plasma glucose, insulin, and acyl-carnitines were also upregulated after blackberry consumption. Antioxidative enzyme system reinforcement correlated with the anti-inflammatory and anti-dyslipidemia potential of blackberry extracts [127]. The glycemic and lipidemic-controlling mechanisms of blackberry extracts were mediated through the downregulation of lipogenesis factors (FAS, SCD-1, microsomal triglyceride transfer protein, diglycerides acyltransferase, and adipose triglyceride lipase), energy coupling/uncoupling proteins (UCP-1, UCP-2, and UCP-3), pro-inflammatory cytokines (PPAR- $\alpha$, Nrf2, IL-6, and TNF- $\alpha$ ), and fatty acid $\beta$-oxidation genes (CPT-1a and ACOX-1) (Table 1) [127], which were maintained by long-term and chronic blackberry extract consumption. Additionally, the increases in total monounsaturated fatty acid contents of adipocytes, plasma brain-derived neurotrophic factor levels, and pro-inflammatory leptin levels in HFD-fed controls were counteracted by blackberry extract consumption [128]. Human clinical trials were also run, in which healthy human subjects were given BB fruits in addition to HFD, resulting in reduced fat accumulation and increased fat oxidation. Blackberry consumption lowered postprandial glucose and lipid levels by activating AMPK and BAdTs. [291]. Pulpy blackberry juice consumption by dyslipidemic patients decreased ApoB and hs-CRP, increased ApoA-1 and HDL-C, and left other lipid parameters unaffected [134]. In healthy subjects, blackberry juice increased exogenous and endogenous antioxidant enzymes. Cyanidin, ascorbate, total ellagic acid, urate, and R-tocopherol contributed to increased plasma and urine antioxidant capacities [125,133]. Daily blackberry consumption reduced dyslipidemia and insulinemic parameters in diabetic and obese adults [132]. Blackberry polyphenolic compounds inhibit digestive enzyme activities, physically interacting with hexose absorption transporters and modulating transporter expression at the genomic level [292]. Blackberry compounds may also modulate peripheral glucose use, damaged pancreatic cell regeneration, and enhance blood glucose withdrawal by increasing insulin sensitivity (Table 1) [109,129].

Blackberry juice was also examined in STZ-induced-diabetic male Sprague-Dawley and hamster rats (Table 1) [129]. Blackberry juice significantly reduced food and water intake, reducing the BWs of both control and diabetic rats [129]. Blackberry nectar supplementation of a cholesterolemic diet reduced hyperlipidemic parameters and hepatic lipid peroxidation [181]. Blackberry juice consumption effectively reduced triacylglycerols $(-43.5 \%)$, glucose $(-48.6 \%)$, and cholesterol $(-28.6 \%)$ levels without side effects. Blackberry juice consumption limited lipid peroxidation in the plasma $(-7.5 \%)$ and kidneys $(-19.5 \%)$. Similarly, alcohol-free fermented blackberry juice (AFBBJ) was used to supplement HFD in obese C57BL/6J mice [270], which significantly reduced fat-mass gain and FBS and decreased plasma TG, TC, LDL-C, and HOMA-IR levels, while increasing $\beta$-cell function (HOMA- $\beta$ ) [22]. Liver function tests revealed no change in ALT, but AST increased in AFBBJ-treated mice. Genomic sequencing approaches revealed pancreatic gene upregulation, responsible for amino acid and glucose metabolism and insulin secretion regulation [22].

The intestinal bioavailability of blackberry polyphenols and resulting impact on gut microflora have also been recently investigated. The low-absorption and cecal accumulation of BB polyphenols were the main reasons for positive health effects. The cecal microbial fermentation of blackberry polyphenols generates antidiabetic and antioxidative blackberry metabolites including C3G, 2,4,6-trihydroxybenzoic acid, coumarin, and caffeic acid. The increased cecal glycoside concentration and secondary metabolites improved glucose consumption (Table 1) [290]. The increased cecal SCFA concentration suggested an increase in SCFA-producing bacteria; however, the relative abundance of different bacterial groups was not reported [130]. Blackberry treatment altered the gut microfloral composition by increasing cecal Bacteriodetes over Firmicutes. Lactobacillus johnsonii was abundant in both blackberry-treated and control groups, whereas Lachnospiraceae dominated the blackberry group, promoting glycoside metabolism. However, Clostridiales, Enterococcus faecalis, and Bifidobacterium pseudolongum were more dominant in the control groups [131]. 


\section{Strawberries}

Strawberry (Fragaria $\times$ ananassa) consumption has been associated with decreased risk and occurrence of metabolic syndrome, cancer, diabetes, chronic inflammation, and hypertension. The credit of these health-promoting activities goes to its rich phytochemical contents (Table 2). Many studies analytically analyzed the crude and fractionated phytochemical contents of strawberry and found strawberry rich in antioxidative, anti-obesity, antiglycation, anti-inflammatory, and antidiabetic compounds from flavanols, flavonols, anthocyanins, hydroxycinnamic acid derivatives, hydroxybenzoic acid derivatives, ellagic acid and ellagic acid glycosides, and ellagitannins (Table 2). The most surplus glucose-lowering acid moieties were malonic and $p$-coumaric acid and the most identified flavonols of strawberry were derivatives of kaempferol and quercetin glycosides. The red-coloration-granting and anti-oxidative anthocyanins of strawberries were mostly the derivatives of pelargonidin and cyanidin [240]. The hydrolysis of ellagitannins gave rise to the most important antidiabetic phytochemical called ellagic acid, which comprised more than $50 \%$ of the total polyphemolic components of strawberry. The level of ellagic acid is about 3-10 times higher in the strawberry than other berries, fruits, and nuts. It is one of the constituents due to which strawberry can regarded as a functional food [293]. In in vitro studies, strawberry ethanolic extracts inhibited pancreatic lipase activity more strongly than reference orlistat. Aqueous and ethanolic strawberry extracts inhibited adipocyte cell division and inhibited inflammatory mediator ( $\beta$-hexosaminidase and histamine) release by 61.8 to $80 \%$ [294]. Strawberry polyphenolic compounds interact with glucose transporters such as SGLTI and GLUT2 and attenuate glucose uptake due to polyphenol compound competition for transporter active sites [295]. HPLC-diode array detector (DAD)-MS analysis and statistical correlations showed the contribution of pelargonidin-3-O-glucoside to glucose uptake inhibition. Strawberry extracts effectively inhibited uptake and transport of glucose up to $5 \%$ in HepG2 cultures [295]. Da Silva Pinto et al. [296] showed that the strawberry extract $\alpha$-glucosidase inhibitory activity was superior to the $\alpha$-amylase inhibitory activity. Strawberry-derived ellagitannin consumption $(>50 \mathrm{mg} / \mathrm{mL}$ ) sufficiently inhibited ACE activity [296]. Methanolic strawberry extracts activated p-AMPK/AMPK expression in HepG2 cells, resulting in fatty acid and cholesterol regulatory gene inactivation and phosphorylation including HMG-CoA-R and ACC. Activated p-AMPK/AMPK expression increased LDL receptor expression including PGC- $1 \alpha$ and sirtuin 1 (a NAD ${ }^{+}$-dependent deacetylase that inhibit hepatic lipogenesis, stimulating FA $\beta$-oxidation, and maintaining cholesterol and bile acid levels) in HepG2 cells [297].

Numerous in vivo studies have also cited the health promoting activities of strawberry or its byproducts in animal models and human clinical trials. The intake of aqueous, alcoholic, and hydro-alcoholic strawberry extracts improved the serum glucose level, liver function (decreased serum glutamic pyruvic transaminase, serum glutamic oxaloacetic transaminase, alkaline phosphatase), lipid profile (decreased LDL-C, LDL-C/HDL-C, and LDL-C/TC ratio), and lipid oxidation markers (decreased MDA and CAT) [136,137]. Genes associated with glucose, cholesterol, and lipid metabolism [FAS, ACC, CPT-1A, malonyl-CoA, acyltransferase, ACC- $\alpha$ (ACACA), and acyl-CoA synthetase long-chain family member 1] were also downregulated by strawberry treatment [135]. Paquette et al. [143] used the hyperinsulinemic-euglycemic clamp methodology to examine improved insulin sensitivity and secretion after strawberry extract consumption, but did not detect improvements in fasting insulin and glucose concentrations. In animal studies, HFD supplementation with strawberry prevented weight gain without influencing food and water intake. Strawberry beverage consumption protected against postprandial lipemia by reducing TG (14\%), TC (5\%), and LDL-C levels (5\%) in hyperlipidemic patients following HFD [144]. Sugar-rich strawberry jam consumption also attenuated glycemic index and postprandial glucose level increases in diabetic human subjects $[145,298]$. Strawberry jam consumption showed favorable lipid and sugar metabolism results, even compared with low-sugar strawberry jam [146]. Strawberry consumption with HCD also controlled postprandial glucose levels, affected glucose and insulin responses, and GLP-1 expression. Regular strawberry beverage and juice consumption decreased blood pressure, TC, and the TC/HDL-C ratio in 
diabetic patients. T2DM and CVD risk factors were also ameliorated (Table 1) $[66,114,123,147]$. Strawberry extracts reduced IL-6 and plasminogen activator inhibitor 1 (PAI-1) (a risk factor for atherosclerosis) levels in obese individuals after HFD/HCD, without influencing TNF- $\alpha, \mathrm{CRP}$, platelet aggregation, or fasting insulin and glucose levels [148]. In another similar study, the postprandial insulin level and inflammatory response (hs-CRP and IL-6) were reduced with increased plasma pelargonidin sulfate and pelargonidin-3-O-glucosidein levels after strawberry powder consumption with high-carbohydrate, moderate-fat meals [149]. In another recent study, strawberry-blueberry powder, consumed with a HFD/HCD, reduced BW gains (12.7\%), visceral fat mass (18\%), retroperitoneal and subcutaneous white adipose tissues (up to 10.45-16.5\%), postprandial insulin and glucose levels, IR, and inflammatory markers (MCP-1, TNF- $\alpha$, IL6, CRP, and PPAR- $\alpha$ ), in male Wistar rats and C57BBL/6J mice (Table 1) [19,138]. Strawberry-blueberry powder exerted anti-adipogenic effects by regulating lipid metabolizing genes including PPAR- $\alpha$ and $C / E B P \alpha$. Inflammatory and lipogenesis-related gene expression were reduced including TNF- $\alpha, \mathrm{IL6}$, and $\mathrm{C} / \mathrm{EBP} \alpha$, adipogenesis-driver transcription factors (PPAR- $\gamma$ ), adiponectin, adipocyte fatty acid-binding protein, SREBF1, leptin, SCD-1, and FAS [138]. In another dose-response checking study, the intake of strawberry against the Western-type-meal reduced the oxidized low-density lipoproteins and post-meal insulin demand in insulin resistant patients [150].

Oxidative stress is a leading cause of metabolic syndrome and diabetes. Strawberry powder supplementation in an isoenergetic diet containing the oxidative-inducing antibiotic drug doxorubicin reversed doxorubicin-induced decreases in the antioxidants retinol and $\alpha$-tocopherol and upregulated liver antioxidant enzymes including GPx, CAT, GSH, SOD, and GST (Table 1). Plasma hepatic stress biomarker levels including protein carbonyls and hydroperoxide were reduced by strawberry intake $[139,152]$. Strawberry-based foods containing carbohydrate, fat, and lipids increased total antioxidant levels (1.26 to $1.45 \mathrm{mmol} / \mathrm{l}$ ) of the subjects while decreasing $\mathrm{HbA1C}$ (from 7.00 to $6.72 \%$ ) levels. The plasma hs-CRP and MDA levels also decreased from 3.36 to $2.76 \mathrm{nmol} / \mathrm{mL}$ and 3.36 to $2.76 \mathrm{nmol} / \mathrm{mL}$, respectively [153]. Strawberry powder intake prevented HFD- and stress-induced decreases in $\gamma$-aminobutyric acid levels and reduced oxidative stress and lipid oxidation markers, in male Wistar rats [140]. Fresh strawberry consumption reduced linseed oil-induced DNA damage and plasma oxidative marker levels and increased the plasma antioxidant status of pigs [299].

Strawberry intake effects on gut microbial ecology in diabetic subjects increased phylogenetic species richness ( $\alpha$-diversity) and global microbial composition ( $\beta$-diversity) variations at the genus and operational taxonomic unit levels. Proteobacteria, Actinobacteria, and Verrucomicrobia were significantly altered after the strawberry intervention. Strawberry intake significantly increased the abundance of beneficial Bacteroides and Actinobacteria and decreased Akkermansia, Verrucomicrobia, Dehalobacterium, and Dorea (Firmicutes). At the genus level, the abundance of SCOA-producing Lactobacillus and "prebiotic-effect-giving" Bifidobacterium increased, whereas Dehalobacterium, Dorea, SMB53, and Turicibacter remained unaltered [141]. Additionally, a specific relationship between ingested flavonoids and microbial community patterns was identified [151]. Dietary flavanol and flavanone intake were positively associated with Eggerthela lenta. Flavonols and flavanol monomer intake was positively associated with Adlercreutzia equolifaciens (involved in phytochemical degradation) and inversely associated with Flavonifractor plauti (Gram-negative poorly understood) populations [151]. Whole strawberry powder intake increased the $\alpha$-diversity of colonic inflammatory CD-1 mice, increasing Bifidobacterium and Lactobacillus and reducing pro-inflammatory Akkermansia, Dorea, and Bilophila [142]. The polyphenolic compounds that affected gut microbiota compositions in strawberry fruit extracts were flavanols, flavonols anthocyanins, hydroxycinnamic acid derivatives, hydroxybenzoic acid derivatives, ellagic acid, ellagic acid glycosides, and ellagitannins (Table 2) [240,241]. 


\section{Goji Berries}

Goji berry (GB, Lycium Barbarum) is a functional food and alternative therapeutic tool for T2DM treatment [155]. The major GB therapeutic phytochemicals include polysaccharides (5-8\%), carotenoids $(0.03-0.5 \%)$, and phenolic compounds (traces). The compounds belonging to these classes have been listed in Table $2[123,242]$. The GB is considered the best source of dipalmitin zeaxanthin carotenoids. These carotenoids showed effective protection against diabetic-induced-retinopathy [300]. The in vitro hypoglycemic tests showed the inhibitory capability of GB carotenoids was 9.6 to $82.6 \%$ and 5.7 to $15.3 \%$ for $\alpha$-glucosidase and $\alpha$-amylase enzymes, respectively [242]. In GB polyphenolic compounds, phenolic acids $(24.7 \%)$ and flavonoids $(75.3 \%)$ are major phytochemical classes. The major therapeutic flavonoids in GB are squercetin-3-O-rutinoside (from 7.1 to $232.7 \mathrm{mg} / \mathrm{kg}$ ) and quercetin-3-O-hexoside (from 169.1 to $1107.7 \mathrm{mg} / \mathrm{kg})$ whereas phenolic compounds include caffeoylquinic acid $(0.34 \mu \mathrm{g} / \mathrm{g})$, caffeic acid $(3.73 \mu \mathrm{g} / \mathrm{g}), p$-coumaric acid $(6.06 \mu \mathrm{g} / \mathrm{g})$, chlorogenic acid $(12.4 \mu \mathrm{g} / \mathrm{g})$, kaempferol-3-O-rutinoside $(11.3 \mu \mathrm{g} / \mathrm{g})$, quercetin-diglucoside $(66.0 \mu \mathrm{g} / \mathrm{g})$, and rutin $(42.0 \mu \mathrm{g} / \mathrm{g})$ [242]. As GB polysaccharides (GBPS) are major contributors of health-endowing activities and have been vastly investigated, this section will primarily focus on GBPS. GBPS are considered to be therapeutic in alternative medicine with immunomodulation, antioxidant, neuroprotection, anti-tumor, antidiabetic, radioprotection, anti-osteoporosis, hepatoprotection, and anti-fatigue activities. The GBPS biological activities depend on their molecular weight, chemical structure, and chain conformation [154,301]. The GBPS are among a few plant-based bioactive compounds that have shown simultaneous hypoglycemic and hypolipidemic properties. Due to hypoglycemic and anti-hyperlipidemic properties, GBPS may be a potent T2DM inhibitor, delaying disease prognosis, even after disease development. Antidiabetic assays showed impressive lipid and glucose reducing effects [155,302]. Acidic GBPS treatment in rat insulinoma cells decreased oxidative stress biomarkers and increased antioxidant enzyme systems. GBPS treatment of IR alloxan-treated-HepG2 cells protected against oxidative stress and improved cell survival and proliferation [302]. Similarly, the GBPS was further checked for possibly hampering glucose uptake in the gut and intestine. The GBPS intensively reduced glucose absorption in a dose-dependent manner by competing for intestinal absorption [303]. Rat insulinoma cells incubated with GBPS rescued damaged pancreatic cells, improved the survival rate, and encouraged insulin secretion. The IR cell model was supplemented with purified GBPS, which upregulated glucose consumption. GBPS was easily translocated and transported across the Caco-2 intestinal cell membrane through the SGLT-1 transporter, producing a hypoglycemic effect. Therefore, GBPS is a plant-based bioactive compound that shows simultaneous hypoglycemic and hypolipidemic properties [303]. Purified GBPS fractions showed dose-dependent hypoglycemic activities, resulting in increased glucose uptake [156,303]. Besides GBPS, GB carotenoids have also shown antidiabetic and $\alpha$-glucosidase and $\alpha$-amylase enzyme inhibitory activities [242].

The hypolipidemic effects of GB intake have been studied by in vivo approaches (Table 1), but human clinical trials for GB have been limited, with most studies performed using small sample sizes in China. GB consumption effectively reduced serum lipid peroxide species in diabetic patients. Reductions in waist circumference, TG, transaminase, and TC levels were reported in metabolic syndrome patients following routine GB intake. Lipid profile improvements were accompanied by increased GSH and CAT enzymatic activities [167]. GB anthocyanins reduced BW gain (17.4 to $38.7 \%$ ) by increasing fecal fatty acid contents and downregulating IL-6, TNF- $\alpha$, IFN- $\gamma$, NF- $\mathrm{kB}$, and iNOS gene expression [157]. GBPS decoction treatment of alloxan-induced, diabetic, obese rabbits effectively reduced blood glucose levels. GBPS substantially decreased serum TG $(-4.27 \%)$, TC ( $-3.5 \%)$, LDL-C levels, and increased HDL-C serum levels (0.78) [154]. The hypoglycemic and hypolipidemic effects of GBPS were later confirmed by the works of Zhao et al. [158]. Supplementation of HFD with GBPS decreased HOMA-IR, fasting and postprandial insulin and glucose levels, serum TG, TC, and LDL-C levels, and weight gain [158].

The oxidative stress relieving effect of GBPS was also checked (Table 1). The effect of GBPS treatment on the kidneys of STZ-induced diabetic rats increased kidney antioxidant enzymes including 
CAT, SOD, GBPx, GST, and GSH [170]. The supplementation of GB in the form of GB milkshakes increased plasma zeaxanthin and antioxidant levels by 57 and $26 \%$, respectively. GB juice (GBJ) also increased GSH peroxidase (GSH-Px) and SOD by $9.87 \%$ and $8.7 \%$, respectively and decreased MDA levels by $5.95 \%$ [166]. GBPS intake also protected against glaucoma, which was confirmed in retinal ganglion cells, and disrupted intraocular pressure [159]. GBPS administration to C57BL/6 mice reversed oxidative stress, dyslipidemia, and diabetic changes. GBPS administration downregulated nitrotyrosine and MDA expression and increased antioxidant enzymes such as CAT, GPx, and $\mathrm{Cu} / \mathrm{Zn}$ SOD. GBPS intake also diminished pro-inflammatory biomarkers including TNF- $\alpha$, IL-1 $\beta$, iNOS, and COX-2. Following pro-inflammatory marker reduction, liver injury biomarkers, called chemokines, were also reduced. The liver regeneration process was also observed following GB intake, enhancing liver regeneration biomarkers [168,304].

With respect to hypoglycemic effect specifically (Table 1), Zhao et al. [160] confirmed the antidiabetic characteristics of GBPS, which increased GLUT-4 expression in the skeletal muscle plasma membrane. Purified GBPS in pancreatic cells increased glucose uptake and metabolism, insulin secretion, and proliferation. The enhanced glucose metabolism mechanism was associated with increased hepatic hexokinase and pyruvate kinase expression/activity (Table 1) [26,161]. GBPS may block the ATP-sensitive $\mathrm{K}^{+}$channel, activate glycogen synthetase and insulin-like growth factor, enhance peripheral glucose utilization, or inhibit glucagon releasing factors in pancreatic $\alpha$-cells [197]. In a recent single meal challenge study, increased glucose and lipid consumption were observed in GB-treated patients, associated with increased respiratory quotients, oxygen usage, and carbon dioxide release. However, no single-dose effects on substrate oxidation and postprandial-energy-expenditure were reported [169]. Du et al. [162] compared GBPS with metformin and reported similar normalization effects on blood glucose and insulin levels. This study also reported reduced IL-2, IL-6, TNF- $\alpha$, intercellular adhesion molecule-1 (ICAM-1), MCP-1, and blood urea/nitrogen levels, inhibited albuminuria, and reversed histopathological alterations. GBPS treatment in HFD/HCD-fed rats also demonstrated hypoglycemic and hypolipidemic effects [115]. Ni et al. [163] examined the potential neuroprotective effects of aqueous GB extracts. Retinal apoptosis causes photoreceptor degradation and diabetic retinopathy (DN), and GB carotenoid supplementation in rats hampered caspase-2-induced apoptosis, protecting photoreceptors [163]. Prolonged or chronic hyperglycemia downregulates luteolin and zeaxanthin-metabolizing gene expression, causing retinopathy. GB carotenoids protected against diabetes-induced retinopathy. GB supplementation upregulated carotenoid metabolism genes and retina biogenesis in STZ-induced diabetic rats [300]. GB also contains taurine, a non-essential amino acid, and GB-derived taurine enhanced PPAR- $\gamma$ activity and elevated cAMP levels, hampering the prognosis of DN with reversal of epithelial barrier impairments [300].

GBPS, polyphenol, and carotenoid effects on the gut microbiome were also studied (Table 1). Fermentation and simulated digestion experiments revealed that GBPS was digested and degraded only in the distal gut, releasing monosaccharides and promoting beneficial SCOA-producing bacterial growth. Monosaccharides with side chains are more susceptible to degradation than monosaccharides with linked backbones. GBPS greatly increased SCFA-producing gut microbiota and increased Bacteroidetes (including Prevotella and Bacteroides) and Actinobacteria (containing Collinsella and Bifidobacterium) populations, whereas Megamonas and Megasphaera (Firmicutes) populations were decreased. Furthermore, SCOA/SCFA-producing, prebiotic-effect-giving, proteolytic microflora such as Bacteroides, Phascolarctobacterium, Bifidobacterium, Prevotella, Clostridium XlVb, Oscillibacter Collinsella, and Lactococcus were prominent following GBPS treatment [305]. In another study, dietary GB supplementation also increased health-promoting secondary metabolite and SCOA-producing Actinobacteria, Lachnospiraceae, Clostridium XIVb, Sporobacter, Pseudoflavonifractor, Butyriccicoccus, Anaerotruncus, Anaerosporobacter, and Ruminococcaceae populations without affecting Akkemansia, Mucispirillum, Bacteriodes, and Desulfovirio. Butyryl-Coenzyme A CoA transferase is an important butyrate gene, and GBPS supplementation increased its expression in butyrate-producing bacteria such as the Clostridium cluster XIVa group including Lachnospiraceae, Faecailbacterium prausnitzii, and 
Ruminococcaceae [164]. The GBPS prebiotic effects increased the populations of Firmicutes, Akkermansia, Proteobacteria, Lactobacillus, and Prevotellaceae [165].

\section{Acai Berries}

Acai berry ( $\mathrm{AB}$, Euterpe oleracea) is native to South America and has high phytochemical contents. The dominant antidiabetic phenolic acid constituents in $A B$ include ferulic acid, anthocyanin-3-glycosides, $p$-hydroxybenzoic acid, epicatechin, protocatechuic acid, gallic acid, ellagic acid, catechin, $p$-coumaric acid, vanillic acid, and gallotannins (Table 2) [246]. Anthocyanin and flavonoids are prominent therapeutic polyphenols including C3G and C3R [244,245]. AB juice (ABJ) is richer in polyphenols and flavonoids than other berry juices, resulting in increased antioxidant capacities [200]. In in vitro studies, the isotonic ABJ pancreatic lipase inhibitory activity was significantly positively correlated with anthocyanin contents. Isotonic $\mathrm{ABJ}$ also reduced adipogenesis and lipid accumulation in 3T3-L1 adipocytes and inhibited $\alpha$-glucosidase activity [306]. Isotonic ABJ also inhibited Cu-mediated LDL oxidation and oxidized or acetylated LDL uptake. AB puree also showed antiglycation activities at a concentration $0.1 \mathrm{mg} / \mathrm{mL}$, which was $89 \%$ stronger than the control [171]. Polyphenols in ABJ affect adipogenesis, preventing obesity, weight gain, inflammation, and diabetes [307].

In in vivo studies, $A B$ fruit proved to be a very useful therapeutic agent for circumventing oxidative stress, and controlling dyslipidemic and metabolic syndrome conditions (Table 1). The supplementation of $A B$ fruit effectively prevented protein oxidation as increased protein sulfhydryl groups were observed, with decreased protein oxidation biomarker carbonyl proteins. A single AB pulp dose enhanced plasma antioxidant capacity 7 -fold $3 \mathrm{~h}$ after its consumption. Plasma anthocyanins reached maximum levels $2.2 \mathrm{~h}$ after $\mathrm{AB}$ pulp consumption [179,180]. In another in vivo study, $\mathrm{AB}$ pulp supplementation in oxidatively damaged mutant Drosophila melanogaster, in combination with HFD, reversed HFD-induced oxidative stress damage and prolonged the lifespan expectancy by $22 \%$ [172,308]. $\mathrm{AB}$ supplementation with exercise improved hepatic oxidation status by reducing inflammatory MCP-1 expression, SOD activity, redox-sensitive signaling pathway activation, ROS generation, and ROS stress [173]. To elucidate the antidiabetic and antioxidative molecular mechanism of $A B$, AB-mediated transcript-level changes were examined in 12 genes associated with JNK, nutrient sensing, and insulin-like signaling pathways [309]. PEPCK genes, involved in glyceroneogenesis and gluconeogenesis, were reduced in the $\mathrm{AB}$ pulp group. Cholesterolemic diet consumption decreased lethal/essential or life gene (lefl2) expression, which was reversed by AB fruit consumption. Two JNK targets, metallothionein $\mathrm{A}$, and glutathione $\mathrm{S}$ transferase $\mathrm{D} 1$, which have antioxidant activities, were upregulated after $\mathrm{AB}$ consumption without affecting the remaining JNK downstream target genes (Ferritin 1 heavy chain homolog, Ice, Heat shock protein 68, and Puckered). Moreover, AB ingestion promoted longevity by intensifying stress response pathway activity and suppressing PEPCK genes $[172,309]$. Treatment with AB seed extracts also reduced blood pressure, the hypertension biomarker renin, and DN biomarker levels (creatinine, urea, creatin, and albumin). Diabetes onset leads to oxidative stress and hypertension, decreasing the number of glomeruli per area per kidney, a major $\mathrm{DN}$ marker. AB seed extracts reduced kidney volume expansion and prevented a decrease in the number of glomeruli per area per kidney [174]. AB seed extracts substantially reduced renal injury (resulting in reduced urea and creatine excretion), hampering renal fibrosis progression. The diabetes-induced glomerular filtration barrier injury markers, podocin and nephrin, decreased in diabetic male Wistar rats, whereas $\mathrm{AB}$ seed extract treatment restored these levels. $\mathrm{AB}$ seed extract treatment also reduced renal proinflammatory cytokines and oxidative stress biomarkers, reinforcing the anti-oxidative defense system [174]. The effects of exercise and AB seed-rendered extract consumption in STZ and HFD-induced diabetic rats reduced HbA1C, glycemia, serum insulin, HOMA-IR, serum TG, TC, LDL-C, and HDL-C levels [176]. Insulin signaling components (insulin receptors, pAKT, and AKT) in skeletal muscles were upregulated following $\mathrm{AB}$ seed extract consumption and exercise [176]. Reduced adiponectin levels are observed in T2DM, associated with deregulated sugar and lipid 
metabolism, and AB seed extracts reversed this effect. AB seeds induced increased GLUT-4 expression and glucose uptake due to AMPK activation [176] and increased GLP-1 and incretin levels with reduced leptin and inflammatory cytokine expression, which were not observed in HFD-fed rats treated with exercise alone. Increased GLP-1 and incretin expression promotes insulin secretion, suppressing gastric emptying, and glucagon synthase [176,310]. The same research group then used the $\mathrm{AB}$ seed extracts to check the anti-obesity features in the C57BL/6 mice strain fed on HFD. HFD supplemented with the AB seed extract prevented weight gain in mice [311]. Adiponectin levels, which are responsible for lipid metabolism, decreased in HFD-fed mice and were restored by AB seed extract supplementation. AB seed extracts increased glucose and lipid metabolizing protein expression including $\mathrm{pAMPK} / \mathrm{AMPK}, \mathrm{pACC} / \mathrm{ACC}, \mathrm{HMG}-\mathrm{CoA}$, and various transporters including ATP-binding cassette sub-family G member 5-ABCG5 and ATP-binding cassette sub-family G member 8-ABCG8, while reducing SREBP-1c expression. Similarly, protein and lipid oxidation products including carbonyl proteins and MDA were reduced by strengthening the anti-oxidative enzyme system [311].

Regarding glucose-lowering effect, recently, the human AB fruit consumption with normal meals decreased FBS and mean plasma insulin levels after one month. Plasma TG, TC, and LDL-C levels, and the LDL-C/HDL-C ratio also decreased, with increased plasma HDL-C levels [181]. The AB consumption with HFD enhanced fecal cholesterol contents, with no influence on low-grade-inflammation biomarkers [113]. Freeze-dried AB fruit pulp reversed the HFD-induced alterations in PEPCK expression [312]. Aqueous ethanolic AB extracts restored mitochondrial complex I function by modulating NADH:ubiquinone oxidoreductase core unit 7 and 8 expression. NLRP3 (a component of inflammasome) and caspase 1/caspase 3/caspase 8 (Interleukin-1 converting enzyme family, which initiates inflammatory response) were downregulated in oxidative-agent-treated macrophages [313]. AB supplementation also interfered with hepatic cholesterolemic metabolism. $\mathrm{AB}$ attenuated the high-cholesterol diet effects by reducing weight gain, TC and LDL-C levels, and key regulatory gene expression associated with the cholesterol biosynthesis pathway including HMG CoA-R, EBP-2, ApoB100, LDL-R, ABCG8, and CYP7A1 [175]. Intensive feeding with freeze-dried AB pulp attenuated HFD-induced hepatic steatosis by improving IR, adiponectin expression, adiponectin receptor 2, SREBP-1c, PPAR- $\alpha$, and its target gene, CPT. Fat accumulating gene expression including UCP-2 and fatty acid translocase were reduced by AB treatment [179]. Both lipid accumulation and oxidation were reduced in zebrafish fed with a high-cholesterol diet, and reduced serum TC, LDL-C, and MDA levels were observed in AB-treated zebrafish [171]. Aside from lipid oxidation inhibition, the $\mathrm{AB}$ intake also prevented amino acid oxidation after $\mathrm{HCD}$, reducing protein carbonyls and sulfhydryl groups, which are important protein damage biomarkers. Reduced arylesterase and PON activities and reduced hepatic ALT, AST, and ALP levels demonstrated improved hepatic operation [175]. AB powder also improved anti-inflammatory mechanisms after HFD by improving glucose intolerance and reducing IL- 6 and TNF- $\alpha$ concentrations in epididymal adipose tissue [312].

A comprehensive study examining $\mathrm{AB}$ intake on the gut microflora is currently lacking. Simulated digestion studies examining $\mathrm{AB}$ polyphenols inhibited the growth of symbiotic and saccharolytic Bacteroides, Prevotella, and Clostridium histolyticum. AB polyphenols showed favorable effects on the intestinal SCFA bacteria population including LAB [178]. Guergoletto et al. [177] noted increased intestinal populations of obesity-protecting bacteria (i.e., Bifidobacterium spp., Eubacterium rectale-Clostridium coccoides group, Bacteroides spp-Prevotella group, and FOS-Raftilose P95). However, AB polyphenols showed no considerable effects on Enterococcus spp and C. histolyticum [177].

Conclusively, AB exerted antidiabetic, anti-obesity, antioxidative, and anti-inflammatory actions by reducing the expression of PPAR- $\gamma$ and its modulators (C/EBP- $\$, C / E B P-\delta$, and other C/EBP family members, Kruppel-like factor, and SREBP1C) Moreover, decreased expression level of transcriptomic factors such as C/EBPß $(-0.41 \%), \mathrm{C} / \mathrm{EBP} \alpha(-0.66 \%)$, Kruppel like factor $(-0.83 \%)$, and SREBP1C $(-0.24 \%)$ were also seen $[125,133]$. AB also reduced the expression levels of lipogenic genes FAS $(-0.5 \%)$, aP2 $(-0.7 \%)$, LPL $(-0.7 \%)$, and FATP1 $(-0.55 \%)$. Low-grade-inflammation biomarkers including leptin and total PAI decreased with increasing anti-inflammatory and anti-adipogenic 
adiponectin levels $[170,172,309,314]$. The expression levels of the pro-inflammatory factors NF- $\mathrm{KB}$, TNF- $\alpha$, MCP-1 (-0.81\%), IL-6 (-0.48\%), IL-8 (-0.05\%), IL-1 $\beta \beta(-0.03 \%)$, and INF- $\beta(-0.49 \%)$ were also reduced. TNF- $\alpha$ activates NF- $\mathrm{KB}$ and interleukins (IL-2 and IL-6), which was prevented by ABJ polyphenols $[170,313,314]$.

\section{Chokeberries}

Chokeberries (black chokeberry (BCB), Aronia melanocarpa, red chokeberry (RCB), Aronia arbutifolia) can be consumed as whole fruit, jam, wine, juice, syrup, tea, soft spreads, chili starters, salsa, beer, extracts, gummies, ice cream, and tinctures. CB consumption was used to treat colds in America and to treat hyperglycemia, metabolic syndrome, and hypertension in Europe and Russia. In in vitro bioassays, $\mathrm{CB}$ extract (CBE) showed significant $\alpha$-glucosidase inhibitory activity compared with the referenced antidiabetic drug acarbose. Purified anthocyanins (cyanidin 3-galactoside, cyanidin 3-arabinoside, cyanidin 3-glucoside, and cyanidin 3-xyloside) were the strongest antidiabetic compounds compared with isolated dimeric and trimeric procyanidins. BCB juice (BCBJ) also inhibited $\alpha$-glucosidase, dipeptidyl peptidase (DPP) IV, and ACE activities by 75, 35, and 95\% in a dose-dependent manner, respectively [182]. BCB fermentation and digestion increase polyphenol bioaccessibility. Fermented and digested Aronia kefir showed stronger $\alpha$-glucosidase $\left(\mathrm{IC}_{50}=152.53 \pm 15.24 \mathrm{mg} \mathrm{kefir} / \mathrm{mL}\right)$ and pancreatic $\alpha$-amylase inhibitory ( $\mathrm{IC}_{50}=146.52 \pm 5.37 \mathrm{mg}$ kefir $/ \mathrm{mL}$ ) activities than non-fermented Aronia $\left(\mathrm{IC}_{50}=365.16 \pm 37048.84 \mathrm{mg}\right.$ and $196.21 \pm 5.50 \mathrm{mg}$, respectively) [315]. BCBJ relieved oxidative stress in $\beta$ TC 3 cells by restoring the anti-oxidative enzyme pool and insulin secretion, as comprehensively explained in Figure 2 [316]. The oxidative-stress-induced reduction in insulin secretion was restored by the BCB extract (BCBE) treatment under basal glucose conditions [316]. BCBE treatment of pancreatic cells nullified cytokine (IL-1 $\beta$ and IFN- $\gamma$ )-induced effects and decreased oxidative stress production [183]. BCBE pretreatment $(0.001,0.01,0.1$, or $1 \mathrm{mg} / \mathrm{mL})$ of diabetic hepatic cells line RINm5F) reduced cytokine-induced-oxidative stress from 19.3-0.39 $\mu \mathrm{M}$ to 14.9-0.35 $\mu \mathrm{M}$ [183]. Similarly, BCBE pretreatment of HAECs nullified the TNF- $\alpha$-induced ICAM-1 and VCAM-1 expression by 35 and $45 \%$, respectively, in a dose-dependent manner. BCBEs also prevented NF- $\mathrm{B}$ p 65 phosphorylation, which activates the pro-inflammatory transcription factor NF- $\mathrm{B}[317,318]$.

Addressing the anti-inflammatory potential of CB, in in vivo clinical studies, Kardum et al. [195,196] administered CBJ to patients with pharmacologically incurable grade I hypertension and high blood pressure, resulting in decreased systolic/diastolic blood pressure, with a stronger effect associated with long-term consumption. CBEs also reduced systolic/diastolic blood pressure [197], particularly in congenital heart disease patients [198]. Following hypertension, inflammation is another diabetes complication and numerous studies have cited the anti-inflammatory potential of BCB or its juice consumption. Increased PPAR- $\gamma 2$ expression was attenuated by BCBEs, reducing downstream lipid metabolizing PPAR- $\gamma 2$ target expression such as PGE receptor and LPL, decreasing intracellular lipid droplet accumulation [184]. Regular BCBJ consumption improved chronic inflammatory conditions, lowering IFN- $\gamma$ and TNF- $\alpha$ levels $[195,196,198]$. The immunomodulatory effects of BCB intake have also been discussed in the literature in STZ-induced male Wistar rats. DM causes immune imbalances because damaged pancreatic cells trigger macrophage and $\mathrm{T}$ lymphocyte infiltration, which lesion $\beta$-cells. BCB consumption by STZ-induced male Wistar rats reduced fibrinogen, TNF- $\alpha$, and IFN- $\gamma$ levels, which returned to their normal values $72 \mathrm{~h}$ post-administration of BCB [199]. 


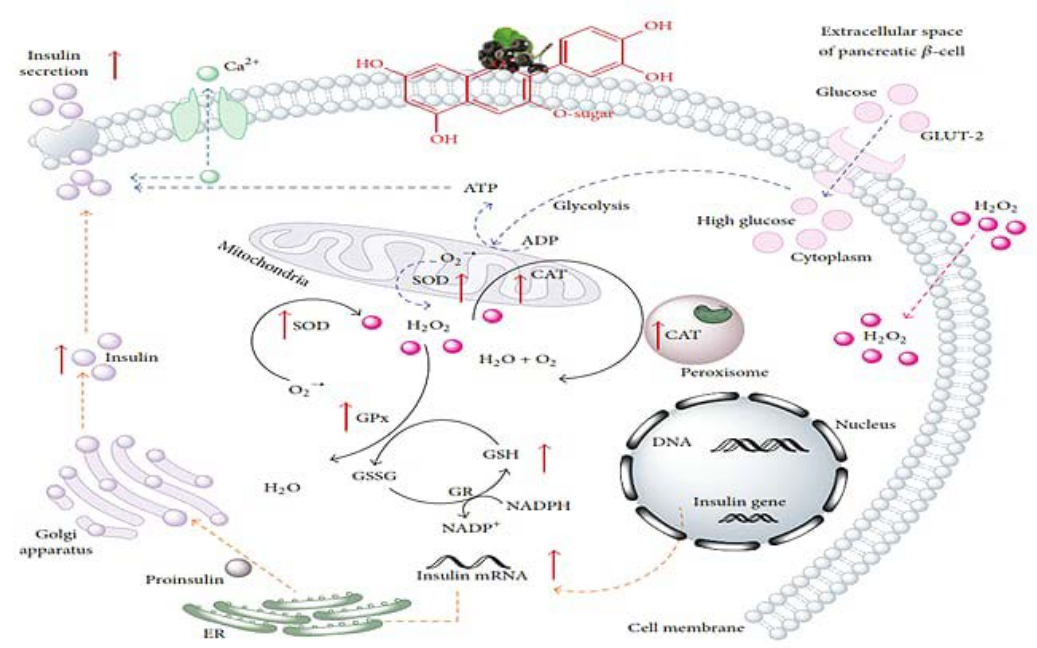

Figure 2. Schematic presentation of chokeberry anthocyanin-induced insulin secretion and antioxidant enzyme pathways in pancreatic $\beta$-cells under high-glucose-induced stress conditions. Glucose is transported across the cell membrane via glucose transporter (i.e., GLUT-2), followed by glycolysis and pyruvate production. Afterward, pyruvate is used for the generation of ATP in mitochondria. Here, in connection with the electron transport chain, radicals, like superoxide anion $\left(\mathrm{O}_{2}{ }^{\bullet-}\right)$, are also produced and simultaneously neutralized by the enzymatic antioxidant SOD. SOD converts the $\mathrm{O}_{2}{ }^{\bullet-}$ into harmless $\mathrm{O}_{2}$ and another radical $\mathrm{H}_{2} \mathrm{O}_{2}$. In addition to $\mathrm{H}_{2} \mathrm{O}_{2}$ diffusion through the cell membrane, $\mathrm{H}_{2} \mathrm{O}_{2}$ is also scavenged by CAT and GPx resulting in water and oxygen production. Chokeberry-derived anthocyanins strengthen this inherent enzymatic antioxidant system (i.e., SOD, CAT, and GPx), which can more actively neutralize the radicals generated during glucose metabolism. $\mathrm{H}_{2} \mathrm{O}_{2}$-stimulated reduction of GSH is also ameliorated by chokeberry anthocyanins. Chokeberry anthocyanins also replenish the pool of insulin by increasing the insulin gene expression. Proinsulin, a precursor of insulin, folded in the endoplasmic reticulum, is transported to the Golgi apparatus. Chokeberry anthocyanins can also influence the opening of the voltage-gated $\mathrm{Ca}^{2+}$ channels, leading to an increased fusion of insulin granules with the cell membrane (Source: Rugina et al. [316]).

Regarding hypoglycemic response, BCBJ consumption also modulated circulating lipid levels including TG, TC, and LDL-C in mild hypertensive patients (Table 1) [185,198]. BCBJ consumption also reduced serum TG, TC, and LDL-C levels in hypercholesterolemic healthy subjects [128]. Long-term BCB consumption was recommended for desirable hypoglycemic and hypolipidemic effects [128,185,198]. Valcheva-Kuzmanova et al. [186] demonstrated up to 39\% reduced postprandial serum TG levels in STZ-induced diabetic rats after BCBJ consumption and reported encouraging results for both diabetic and healthy rats. However, Lipińska and Jóźwik [187] showed pronounced hypolipidemic effects only in diabetic Polish Merino lambs including significantly decreased serum LDL-C and increased HDL-C levels, without significant effects on serum TC levels. In addition to preventing increased plasma glucose, homocysteine, and fibrinogen levels, reduced serum lipid levels (TG, TC, and LDL-C) were observed in STZ-induced diabetic rats [201]. Hepatic steatosis and NAFLD were prevented by BCB treatment in HFD-fed diabetic C57BL/6N mice. Daily BCBE administration prevented increased body, liver, and epididymis weights [188]. Several possible mechanisms have been proposed in the literature referring to the lipid-lowering property of BCB consumption. The BCB hypoglycemic effect may be associated with increased cynidine-induced lipid metabolism, reduced catechin-induced cholesterol absorption, and the flavonoid-influenced downregulation of cholesterol synthesis enzymes including HMG-CA-R, cholesterol acyltransferase, and acyl-CoA $[185,188]$.

The anti-oxidative, anti-obesity, and anti-diabetic potential of BCB was checked in the various diabetic model mice (Table 1), where BCB increased serum insulin secretion with reduced pro-inflammatory cytokine expression (MAPKs, NF-кB, COX-2, and iNOS) in a dose-dependent manner [183]. Jurgoński et al. [189] fed BCBE to high-fructose-diet-fed STZ-induced diabetic rats and 
showed increased maltase and sucrase activity, and decreased lactase production in the small intestinal mucosal membrane. Daily BCBJ consumption lowered postprandial glucose levels after OGTT, regardless of gender, and reduced ACE, $\alpha$-glucosidase, and DPP IV activities in a dose-dependent manner [182]. Valcheva-Kuzmanova et al. [186] showed lower postprandial glucose levels (up to 44\%) in STZ-induced diabetic rats after BCB consumption, and Lipińska and Jóźwik [187] demonstrated a pronounced FBS decrease in BCB-treated Polish merino lambs. Postprandial OGTT results for BCB-treated mice decreased, with improved intraperitoneal ITT results [185]. Similarly, consumption by STZ-induced diabetic mice reduced serum TBARS levels and mitigated lipid peroxidation (by 29-50\%) and kidney hypertrophy [190]. Following $\mathrm{CCl}_{4}$ administration, the decreased concentration of CAT, GPx, and GR were increased by $117 \%, 56 \%$ and $44 \%$, respectively, after the intake of BCBJ. Protein carbonyls, protein oxidation biomarkers, decreased by $22 \%$ after BCBJ consumption in male Wistar rats [190]. BCBJ consumption by the KK-Ay and C57BL/6JmsSlc mice reduced BW, white adipose tissue weight, $\alpha$-glucosidase and DPP IV activity, and blood TG levels. Mesenteric, epididymal, subcutaneous, and retroperitoneal white adipose tissue weights were reduced by 26\%, 27\%, 48\%, and 38\% compared with those in control animals [39]. Bhaswant et al. [191] administered BCBJ to male Wister HFD- and HCD-fed rats and observed reduced BW gain and feed conversion efficiency. Total body fat mass, BMI, abdominal fat (epididymal, omental fat pads, and retroperitoneal), and visceral adiposity index reductions were more pronounced in Wistar rats fed with BCBJ than in those fed with biofunctional purple maize flour. BCBJ consumption also reduced liver injury biomarkers (ALP, AST, and ALT), although these levels remained within the normal range [191]. In another study, male Wistar rats were fed high-fructose diets containing BCBE, resulting in increased plasma HDL-C and adiponectin levels [192]. IRS-1/2 and PI3K regulatory subunit protein expression increased by 2.3-, 1.8-, and 1.5-times, respectively, along with inhibiting the phosphatase and tensin homolog (Pten) $(-0.61 \%)$ expression. The expression level of glucose uptake, transportation (GLUT1 and GLUT4) and gluconeogenesis (GYS) was uplifted by 1.5 times compared to high-fructose fed control rats. BCB consumption inhibited lipogenesis and lipid accumulation by reducing fatty acid-binding protein, FAS, and LPL (lipogenesis protein) by $0.6-0.7 \%$. Improved glucose and lipid metabolism and increased glucose and lipid regulatory metabolizing protein expression (adiponectin and PPAR- $\gamma$ ) were also observed [192]. Cynidine 3, 5-diglucoide was identified as a DPP IV inhibitor. DPP IV cleaves incertins including GLP-I and glucose-dependent-insulinotropic polypeptide at their N-terminal regions, resulting in decreased insulin secretion $[182,186]$. Cyanidin glycosides including 3-galactoside, 3-glucoside, cyanidin 3-O- $\beta$-glucoside3-arabinoside, and 3-xyloside enhance glucose uptake and GLUT4 translocation. Diabetes-associated hyperlipidemic complications were improved by regulating the FOXO1-mediated adipose TG lipase transcription [185].

BCB contains high levels of anthocyanins (1958.18 mg/100 g FW), proanthocyanidins (522-1002 mg/100 g FW), and hydroxycinnamic acids (187.9 mg/100 g FW) including chlorogenic acid and neochlorogenic acid [212,247]. Cynidine-3-O-glucoside, cynidine-3-O-galactoside, cynidine-3-O-xyloside, and cynidine-3-O-arabinoside are the primary antidiabetic and anti-oxidative anthocyanin compounds in BCBJ (Table 2). No studies have examined the CB consumption effects on gut microflora in diabetic/obese individuals, although $\mathrm{CB}$ consumption has been examined in healthy individuals [202]. Chronic BC capsule treatment influenced the intestinal diversity of health promoting and SCOA-producing Anaerostipes, Bifidobacterium, Faecalibacterium, and Clostridium genera. CBE capsules increased the relative abundance of Anaerostipes, whereas whole CB capsules increased Bacteroides and Clostridium XiV populations. Correlation analysis between gut microbial genera and plasma polyphenolic contents revealed that Prevotella, Dialister, Desulfovibrio, and Bifidobacteria were responsible for the increased levels of nine, eight, seven, and six health promoting plasma CB metabolites, respectively, including derivatives of benzoic acid, hippuric acid, phenylacetic acid, cinnamic acid, caffeic acid, flavonols, (iso)ferulic acid, benzaldehydes, and pyrogallol [202]. 


\section{Black Currants}

Black currant (BCT, Ribes nigrum L.) is cultivated primarily in Europe, New Zealand, and Australia. $\mathrm{BCT}$ is a rich source of anthocyanins that represent $95 \%$ of polyphenolic compounds, with the remaining $5 \%$ including other minor polyphenol classes. Delphinidin-3-rutinoside (D3R) is the major $\mathrm{BCT}$ antidiabetic anthocyanin compound that improves glucose tolerance. In BCT nectar, cynidine and delphinidin rutinosides are the dominating anthocyanins, followed by glucoside compounds [93,319]. A full list of other therapeutic BCT compounds are presented in Table 2. GLP-1 and AMPK are the primary BCT polyphenolic compound targets. BCT extract (BCTE) consumption increased GLP-1 secretion. GLP-1, an incretin, promotes pancreatic $\beta$-cell division and glucose-dependent insulin release $[212,213,289]$. BCTEs contain approximately $70 \%$ anthocyanins (especially rutinosides and glucosides of delphinidin and cyanidin) and are considered to be effective $\alpha$-glucosidase inhibitors [289]. Apple and BCT juice (BCTJ) treatment in human Caco-2 cells reduced sodium-independent and total glucose uptake by 46 and 51\%, respectively. In oocytes, apple and BCTJ-derived phloretin and phlorizin effectively reduced glucose uptake by 58 and $85 \%$, respectively [213]. The BCT polysaccharide BCP-I also showed remarkable antiglycation activities due to its inhibitory effects on Amadori products [320]. BCT powder incorporation into high-glycemic-indexed food decreased glucose release and increased antioxidant capacities [321].

In addition to in vitro studies, glucose and lipid lowering effect of $\mathrm{BC}$ extracts or its screened anthocyanins have also been investigated enormously in various in vivo studies (Table 1). The intake of major BCT anthocyanin consumption, in combination with intraperitoneal glucose administration, prevented increased serum glucose concentrations with the simultaneous increase in serum insulin levels [203]. Improved hyperglycemia and hypoinsulinemia are caused by the GLP-activation-induced increase in insulin secretion. BCT powder, administered for six days before OGTT, improved postprandial plasma insulin and glucose levels in healthy human subjects [214]. BCTE consumed with a normal diet by KK-Ay mice induced hypoglycemia and modulated basal GLP-1 concentrations without affecting plasma insulin levels, food intake, or BW [204]. Proglucagon cleaving agent proprotein convertase subtilisin/Kexin type 1, which processes proglucagon into GLP-1, increased. BCTE treatments also increased AMPK phosphorylation in skeletal muscles, upregulating insulin-independent glucose uptake pathways by increasing downstream target expression including GLUT-4 and the translocating plasma membrane [204]. Previously, Esposito et al. [205] also conducted an anti-diabetic study using $1 \%$ BCT powder, which decreased rat BWs, irrespective of dietary fat contents. Microbiological fecal analyses showed increased fecal anthocyanin contents, especially in lean animals. These results suggested that gut microflora more actively transform polyphenolic metabolites in lean animals rather than in obese animals. BCT supplementation reversed the postprandial glucose levels associated with HFD; however, the postprandial glucose level continued to rise due to gut microbiota disruption. Similarly, BCT improved HFD-induced insulin, but the gut microflora disruption increased IR. These results signified the importance of gut microflora during the BCT polyphenol metabolization and biotransformation [205]. The supplementation of 0.1\% BCTE in HFD reduced retroperitoneal and epididymal adipose fat. BCTE hypolipidemic characteristics were verified by upregulated lipogenic/lipid metabolizing genes in adipocytes including UCP-2, UCP-3, mitochondrial transcription factor A (TFAM), PPAR- $\alpha$, SREBP-1c, FAS, and SCD-1, and fatty acid oxidation genes including CPT- $1 \alpha$ and $1 \beta$ [206]. Repressed inflammatory marker expression in macrophages has also been reported. Reduced IKKE (an enzyme complex that is involved in propagating the cellular response to inflammation) and TANK-binding kinase 1 (a member of IKK subfamily, which activates in response to lipopolysaccharides) expression was observed in the BCT-treated group, compared with upregulation in the HFD group [206,212]. BCTJ/nectar waste extract (pomace) was much richer in anthocyanins than in BCT pulp. Phytochemically, BCT pomace extracts are rich in D3G, D3R, cyanidin-3-rutinoside, glycosides, and flavonol aglycones. HFD supplemented with BCT pomace extracts did not affect food intake or BW. Fat in the diet increases small intestinal digesta viscosity, whereas BCT pomace polyphenolic extracts made this digesta more acidic [207]. The polyphenolic-rich 
BCTE also reduced cecal tissue mass and increased ammonia contents. HFD reduced bacterial glycolytic enzyme activities such as $\alpha$ - and $\beta$-galactosidases and $\alpha$ - and $\beta$-glucosidases, which were restored by BCT pomace extract. BCT supplementation reduced $\beta$-glucuronidase activity, which is associated with reduced pressure on the intestinal detoxification mechanism [208]. BCT supplementation reduced the cecal putrefactive SFCA concentration, regardless of diet $[207,208]$. BCTE consumption increased mean fat oxidation during prolonged cycling exercise by endurance-trained females with reduced mean carbohydrate oxidation [215]. However, the opposite outcome was observed when BCTJ was consumed before exercise, without significant effects on blood lactate, glucose, and MDA levels [216].

In addition to HFD, the high-fructose-diet or HCD were also involved in the hyperglycemic, hyperlipidemic, and metabolic syndrome conditions. BCTE administration with high-fructose-diet prevented increases in liver weight, BW, and epididymal fat pad weight. OGTT results improved, with decreased p-AMPK and IRS-1 levels in the BCTE-treated group. BCTE supplementation also decreased high-fructose-diet-induced hyperglycemic marker expression and reduced atherosclerosis risk by diminishing ICAM-1, VCAM-1, E-selectin, endothelin, and eNOS expression levels in aortic tissues [209]. Consumption of an anthocyanin-rich sugar-free BCT drink with a normal-carbohydrate diet delayed the glycemic and insulinemic response with reduced incretin and GLP-1 expression [212,213]. The consumption of BB, BCT, CrB, and strawberries restricted post-meal blood insulin and glucose fluctuations induced by HFD/HCD. LB combined with BCT (whole or nectar) ameliorated postprandial insulinemic and glycemic control and response $[62,123,267]$. The irreversible hydrolysis of sucrose into fructose and glucose under high temperature and low $\mathrm{pH}$ conditions produces invertase sugars. BCT nectar, sweetened with invertase sugars, reduced postprandial blood glucose levels and the maximal blood glucose level by 33 and $87 \%$, respectively. The nectar $x$ time interaction also revealed lower insulin secretion at 15 and $30 \mathrm{~min}$ of post-nectar-consumption and expulsion of insulin from the baseline was cut by $13 \%$ compared to the reference $[62,123,267]$.

Regarding oxidative stress and diabetes-related complications, ample amounts of evidence have suggested that anthocyanins from BC exert anti-hypertensive, anti-inflammatory, anti-fibrotic, and anti-hepatic steatosis effects by limiting lipogenesis and gluconeogenesis (Table 2) [217]. BCT-derived purified extracts administered to hepatic steatosis model C57BL/6J mice did not prevent BW loss, but serum ALT and AST levels increased. BCT anthocyanin supplementation decreased hepatic TG and TC accumulation [304]. Histological analysis showed that microvascular steatosis, inflammatory cell infiltration, and hepatocyte ballooning were reduced by (up to $50 \%$ ) BCT anthocyanins. Hepatic stellate cells produce collagen during fibrogenesis. Reduced $\alpha$-smooth muscle actin and upregulated carbamoyl phosphate synthase 1 suggest hepatic stellate cell inhibition, inhibiting fibrosis and non-alcoholic hepatic steatosis. BCTE treatment increased mitochondrial biogenesis and decreased the AMPK/pAMPK ratio and pivotal mitochondrial biogenesis regulators including PGC- $1 \alpha$ and $\beta$, Nrf- 1 and -2 , and TFAM. Mitochondrial fatty acid $\beta$-oxidation occurs due to mitochondrial oxidative phosphorylation, which was reversed through effects on PPAR- $\alpha$, CPT-1, and medium-chain acyl CoA dehydrogenase expression $[62,93,123,217,304,319]$.

\section{Maqui Berries}

Maqui berries (MB) (Aristotelia chilensis) have recently gained attention due to their high content of polyphenolic compounds. The stated phytochemical composition of MB was $138 \pm 0.4 \mathrm{mg} / 100 \mathrm{~g}$ fresh weight with 35\% relative abundance of delphinidin [218]. Di Lorenzo et al. [219] analyzed the MqB composition (Table 2). MqB is rich in anthocyanins including $84 \%$ diglycosylated and $16 \%$ monoglycosylated anthocyanins $[251,322,323]$. The in vitro sugar hydrolyzing enzymes inhibitory activities of MB extracts were reported by Rubiliar and his colleagues. Rubiliar et al. [324] reported $\alpha$-amylase and $\alpha$-glucosidase inhibitory activities, resulting in decreased postprandial glucose levels and improved glucose tolerance [324]. Crude and purified MqB extracts (MqBEs) reduced MDA production and minimized oxidative damage [250]. An isotonic soft drink containing lyophilized MqB, acai, and blackthorn berry powders [152] demonstrated pancreatic lipase and $\alpha$-glucosidase inhibitory activities, 
which were superior to the control, acai-, and blackthorn-based beverages. Likewise, the in vitro anti-diabetic assay showed the inhibition of $\alpha$-glucosidase activity by $90 \%$ compared to the lemon juice control $(80 \%)$, whilst the recorded inhibitory $\alpha$-glucosidase activity of tested commercial isotonic drinks was around 50\% [306]. The MqBE anti-diabetic and anti-lipidemic potentials were further examined in RAW264.7 mouse monocytes and 3T3-L1 mouse pre-adipocytes [220,325]. MBEs reduced adipocyte formation by promoting MMP-2 and MMP-9 (endopeptidases). GST treatment decreased GSH, SOD, and CAT expression, which was reversed by MqBE treatment in macrophages. LPS treatment increased IL-6, MCP-1, TNF- $\alpha$, and galectin-3 with decreased adiponectin expression, which was countered and reversed by MqBEs in macrophages [220]. Furthermore, a dose of 100 and $180 \mu \mathrm{M}$ MqB delphinidin inhibited sodium palmitate-induced-TG-accumulation by 50 and 59\%, respectively, in Hep2G cells [222].

With respect to in vivo antidiabetic and anti-obesity potential of MqB (Table 1), Rojo et al. [220] fed C57BL/BJ mice anthocyanin-rich MqBEs, which significantly decreased plasma glucose levels following glucose ingestion. Anthocyanin-rich MqBEs also reduced G6Pase and increased insulin sensitivity. Glucose uptake was upregulated in L6 skeletal muscle cells, without toxic effects [220]. Delphinidin 3-sambubioside-5-glucoside, a signature MqB biomarker, showed an equivalent capacity to metformin for normalizing blood glucose levels [326]. Lipid accumulation was inhibited by 4-11\% by MqBE treatment in 3T3-L1 mice; however, lipogenesis was inhibited by 6-38\% during adipocyte differentiation. The lipogenesis inhibitor protein, preadipocyte factor 1 , was upregulated in MqB-treated 3T3-L1 mice. MqB supplementation also exerted an anti-inflammatory response by reducing ROS expression by 9.8 to $61.8 \%$. The expression of COX-2 and production of PGE2 was also evaluated in the RAW 264.7 macrophages to understand the anti-inflammatory mechanism of MqB. MqB inhibited PGE2 expression and reduced COX-2 expression (by 16.2-62\%), inhibiting LPS-induced iNOS/NO production and COX-2/PGE2 pathway activation in macrophages [218,326]. MqB delphinidin anthocyanins inhibited glucose uptake and transport from the rat duodenum by inhibiting SGLT-1. The inclusion of MqB-derived 35\% anthocyanins and 25\% delphinidin glycosides in a rice-chicken diet effectively reduced postprandial glucose levels. Purified delphinidin anthocyanin supplementation with a normal diet reduced fasting glucose and insulin levels [218,326]. MqB anthocyanins, in capsular form $(3 \times 150 \mathrm{mg}$ per day), decreased oxidized LDL-C and 8-iso-prostaglandin F2 $\alpha$, a urinary excretion oxidative stress marker [221,326]. Furthermore, MqB-derived-delphinidin treatment effectively increased AMPK phosphorylation. Gene expression analysis showed that sodium palmitate exposure upregulated lipid accumulating genes such as SREBF1, CPT1-A, patatin-like phospholipase domain containing 2, and FASN, which were reduced by delphinidin treatment. Delphinidin supplementation limited weight gain in HFD-fed C57BL/6Nhsd mice, but not increased liver weight. Glucose homeostasis variations induced by HFD/HCD were also minimized by delphinidin treatment [222]. Hidalgo et al. [327] showed that delphinidin supplementation in rat jejunum tissues/cells reduced the short circuit current generated by glucose addition to an Ussing chamber. Delphinidin halted 3-O-methyl-glucose incorporation in the mouse intestine, with effects similar to the inhibition of electrogenic glucose transportation by SGLT-1 [328]. In response to delphinidin treatment and FFA1 activation, the G $\alpha$ q/11 subunit was coupled with inositol trisphosphate, propionyl 1-carnitine, and diacylglycerol upregulation, which modulates intracellular $\mathrm{Ca}^{2+}$ from the endoplasmic reticulum. In previous studies, delphinidin treatment also caused intracellular $\mathrm{Ca}^{2+}$ release and prevented 3-O-methyl-glucose uptake by FFA1 activation. Therefore, delphinidin may represent a new ligand class that can reduce intestinal glucose uptake through FFA1 activation and increased cAMP expression [327].

MqB juice (MqBJ) consumption limited oxidation in human subjects (Table 2). The copper-triggered LDL-C oxidation time lag increased with MqBJ consumption because anthocyanins chelate copper. LDL-C oxidation time is proportional to the MqBJ anti-oxidative capacity. $\mathrm{H}_{2} \mathrm{O}_{2}$ treatment-induced increased oxidative stress was reduced by MqBJ treatment in human umbilical vein endothelial cells [329]. A pilot study showed that the daily MqBE consumption with folic acid and berberine 
effectively reduced TC, LDL-C, oxidized cholesterol glycemia, free radical levels, and increased serum antioxidant capacity. Furthermore, the insulinemia, microalbuminuria, HDL, CRP, and TG values increased. MqB treatment counteracted hyperlipidemia, hyperglycemia, and ROS production in metabolic syndrome patients. An MqB polyphenol-based-nutraceutical reversed low-grade-inflammation, oxidative stress, and atherosclerogenesis in pre-diabetic patients [223]. $\mathrm{MqBE}$ and purified anthocyanin consumption showed positive outcomes for post-stroke stress and depression in diabetic mice. MqBEs and anthocyanins can mitigate anhedonia in humans. Anhedonic mice consumed less sucrose with increased water intake, which was mitigated by MqBE or purified anthocyanins in a dose-dependent-manner. Stroke and stress biomarkers such as TBARS, SOD, CAT, and GSH levels decreased following MqBE/anthocyanin treatment in stroke model mice [219].

\section{Conclusions}

This review aimed to collect and discuss scientific evidence regarding the positive role of berry consumption on the prevention of diabetes and its complications. Available human, animal, and in vitro studies were collected and comprehensively presented. This review demonstrated that berry product consumption represents a reliable and effective method for preventing and managing metabolic hyperglycemic and hyperlipidemic conditions. Variations in postprandial glucose and insulin levels could be reversed and normalized in diabetic subjects following post-meal berry consumption as supplements for HFD/HCD. Berry anthocyanins promoted glucose uptake and metabolism by activating pAMPK/AMPK, GLUT-4, and SGLUT-1, and inhibited weight gain and pro-inflammatory responses, downregulating lipogenesis genes (adipogenic transcription factors and PPAR- $\gamma 2$ ) and pro-inflammatory cytokine production. Berry consumption also showed glucose-lowering and insulin sensitivity improvements, which are closely associated with hypoinsulinemia, insulin signaling activation (in adipose and skeletal muscles), the adiponectin-AMPK pathway, and GLP-1 upregulation. Regarding the relation of gut microbial ecosystem and DM, berry intake not only counteracted the deleterious HFD/HCD effects, but also favored the population of health promoting fermentative, SCOA/SCFA-producing, obesity-preventing, glycolytic, proteolytic, and secondary metabolites metabolizing microflora. The primary potential health-promoting classes of bioactive compounds found in berries include glycosides, glucosides, catechins, epicatechins, proanthocyanidins, cynidines, delphinidins, quercetin, myricetin, malvidins, petunidin, flavanols, flavonols, caffeic acids, chlorogenic acids, phenolic acids, ferulic acids, $p$-coumaric acids, vanillic acids, ellagic acids, hydroxycinnamic acid derivatives, and polysaccharides. Based on the reviewed papers, to obtain these health-endowing effects, the daily recommended dose of whole berry varies from 200 to $400 \mathrm{~g}$ of berry intake for a $70 \mathrm{~kg}$ BW middle aged person.

Author Contributions: M.C. supervised the work. A.H. planned, wrote, and drafted the manuscript. M.G. assisted with the literature search and the interpretation of data in tabular form. A.K., M.C., and E.A.-P. reviewed and edited the article. All authors have contributed to either the planning of the study or the writing of the article. No author has any potential competing interests to declare. All authors have read and agreed to the published version of the manuscript.

Funding: This research received funding from the European Union's Horizon 2020 Research and Innovation Program under the Marie Skłodowska-Curie grant agreement No. 754432 and the Polish Ministry of Science and Higher Education from financial resources for science in 2018-2023 granted for the implementation of an international co-financed project. The APC was funded by European Union's Horizon 2020 Research and Innovation Program (Research project number: SUB/1/DN/20/009/1196) and Medical University of Bialystok's strategy of excellence project (Research project number: ANZ-0600-SDUB/2/19).

Conflicts of Interest: The authors declare no conflict of interest.

\section{Abbreviations}

1-Deoxynojirimycin (1-DNJ); Acai berry (AB); acetyl coenzyme A carboxylase (ACC); acyl-CoA oxidase (ACOX); adhesion molecules nuclear factor $(\mathrm{I} \kappa \mathrm{B} \alpha)$; advanced glycation end-product (AGE); albumin retention (AR); alcohol-free fermented blackberry juice (AFBBJ); alkaline phosphatase (ALP); aminotransferase (ALT); AMP-activated protein kinase (AMPK); angiotensin I-converting enzyme (ACE-1); apolipoprotein A (Apo)A-I; 
aspartate aminotransferase (AST); ATP-binding cassette (ABC); ATP-binding cassette transporter (ABCA1) Bilberry juice (BBJ); BB/BB extracts, (BBEE); Bilberries, (BBs); Black currant (BCT); BlB extracts (BlBEs); BlB juice (BlBJ); Blueberries (BlBs); body weights (BW); brown adipose tissues (BAdT); capillary albumin filtration (CAF); carbohydrate response element-binding protein (ChREBP); cardiovascular disease (CVD); carnitine palmitoyl transferase-1 (CPT-1); Cranberries (CrBs); cranberries juice (CrB-JSB); CrB extracts (CrBE); CrB extracts (CrBEs); CrB juice (CrBJ); C-reactive protein (CRP); Diabetes mellitus (DM); diabetic retinopathy (DN); diacylglycerol acyltransferases 2 (DGAT2); fasting blood sugars (FBS); fatty acid synthase (FAS); Food and Agriculture Organization of the United Nations (FAO); forkhead box O1 (FOXO1); free fatty acid (FFA); gamma-glutamyltransferase ( $\gamma$-GT); gastrointestinal-digested BB slurry (GIDBB); GB polysaccharides (GBPS); glucagon-like peptide-1 (GLP-1); glucose tolerance test (GTT); glucose transporter (GLUT4); glucose transporter 2 (GLUT-2); glucose-6-phosphatase, (G6Pase); glucose-stimulated insulin secretion (GSIS); glutathione (GSH); glycerol-3-phosphate acyltransferase (GPAT); glycogen synthase (GYS1); glycogen synthase 2 (GSY2); Goji berry (GB); high-carbohydrate diets, (HCD); high-fat diets, (HFD); high-sensitivity CRP (hs-CRP); Human aortic endothelial cells, (HAECs); human serum albumin (HSA); inducible nitric oxide synthase (iNOS); insulin receptor substrate-1/2 (IRS-1/IRS-2); insulin resistance, (IR); intercellular adhesion molecule-1 (ICAM-1); intestinal mucosal barrier dysfunction, (IMBD); lactate dehydrogenase (LDH); Lingonberry (LB); lipopolysaccharides (LPS); Low-calorie dried cranberry, (LCDC); low-density lipoprotein cholesterol (LDL-C); lysophosphatidylcholine (LPC); lyso-phosphatidylethanolamines, (LPE); malondialdehyde (MDA); manganese superoxide dismutase, (Mn-SOD); Maqui berries (MB); microbial-fermented blackberry metabolites (GMBB); microsomal TG transfer protein (MTP); mitochondrial transcription factor A (TFAM); monocyte chemo-attractant protein-1 (MCP-1); Mulberries (MBs); Na-glucose co-transporter 1 (SGLT-1); nitric oxide (NO); nitric oxides (NOs); nod-like receptor pyrin containing 3 (NLRP3); non-alcoholic fatty liver disease (NAFLD); paraoxonase-1 (PON-1); peroxisome proliferator response element (PPRE); peroxisome proliferator-activated receptors $\gamma$ (PPAR- $\gamma$ ); phosphatidylcholines (PC); polyunsaturated fatty acid (PUFA); PPAR- $\gamma$ coactivator $1 \alpha$ (PGC-1 $\alpha)$; proinflammatory nuclear factor (NF)- $\mathrm{KB}$; Raspberries (RBs); RB extracts (RBE); reactive oxygen species (ROS); short-chain fatty acids, (SCFA); short-chain organic acids, (SCOA); soluble vascular cell adhesion molecule-1 (sVCAM-1); sphingomyelins (SM); sterol regulatory element-binding protein 1c (SREBP-1c); streptozotocin (STZ); Toll-like receptors, (TLR); total cholesterol (TC); total glyceraldehyde (TG); Trolox equivalent antioxidant capacity (TEAC); Type 1 diabetes mellitus, (T1DM); type 2 diabetes mellitus, (T2DM); unsweetened dried CrBs (USCB); white bread (WB); World Health Organization, (WHO).

\section{References}

1. World Health Organization (WHO). Organisation for Economic Co-Operation and Development (OECD); World Health Organization: Geneva, Switzerland, 2016.

2. Nathan, D.M.; Buse, J.B.; Davidson, M.B.; Heine, R.J.; Holman, R.R.; Sherwin, R.; Zinman, B. Management of Hyperglycemia in Type 2 Diabetes: A consensus algorithm for the initiation and adjustment of therapy: A consensus statement from the American Diabetes Association and the European Association for the Study of Diabetes. Diabetes Care 2006, 29, 1963-1972. [CrossRef]

3. Enwere, O.; Salako, B.; Falade, C. Prescription and cost consideration at a diabetic clinic in Ibadan, Nigeria: A report. Ann. Ib. Postgrad. Med. 2010, 4. [CrossRef]

4. Evans, D.B.; Etienne, C. Health systems financing and the path to universal coverage. Bull. World Health Organ. 2010, 88, 402. [CrossRef] [PubMed]

5. Wang, D.D.; Hu, F.B. Precision nutrition for prevention and management of type 2 diabetes. Lancet Diabetes Endocrinol. 2018, 6, 416-426. [CrossRef]

6. Ley, S.H.; Hamdy, O.; Mohan, V.; Hu, F.B. Prevention and management of type 2 diabetes: Dietary components and nutritional strategies. Lancet 2014, 383, 1999-2007. [CrossRef]

7. Mursu, J.; Virtanen, J.; Tuomainen, T.P.; Nurmi, T.; Voutilainen, S. Intake of fruit, berries, and vegetables and risk of type 2 diabetes in Finnish men: The Kuopio Ischaemic Heart Disease Risk Factor Study. Am. J. Clin. Nutr. 2013, 99, 328-333. [CrossRef]

8. Moher, D. Preferred reporting items for systematic reviews and meta-analyses: The PRISMA statement. PLoS ONE Med. 2009, 6, e1000097.

9. Barberis, A.; Garbetta, A.; Cardinali, A.; Bazzu, G.; D'Antuono, I.; Rocchitta, G.; Fadda, A.; Linsalata, V.; D'Hallewin, G.; Serra, P.A.; et al. Real-time monitoring of glucose and phenols intestinal absorption through an integrated Caco-2TC7cells/biosensors telemetric device: Hypoglycemic effect of fruit phytochemicals. Biosens. Bioelectron. 2017, 88, 159-166. [CrossRef]

10. Martineau, L.C.; Couture, A.; Spoor, D.; Benhaddou-Andaloussi, A.; Harris, C.; Meddah, B.; LeDuc, C.; Burt, A.; Vuong, T.; Le, P.M.; et al. Anti-diabetic properties of the Canadian lowbush blueberry Vaccinium angustifolium Ait. Phytomedicine 2006, 13, 612-623. [CrossRef] 
11. DeFuria, J.; Bennett, G.; Strissel, K.J.; Perfield, J.W.; Milbury, P.E.; Greenberg, A.S.; Obin, M.S. Dietary blueberry attenuates whole-body insulin resistance in high fat-fed mice by reducing adipocyte death and its inflammatory sequelae. J. Nutr. 2009, 139, 1510-1516. [CrossRef]

12. Wu, T.; Tang, Q.; Gao, Z.; Yu, Z.; Song, H.; Zheng, X.; Chen, W. Blueberry and mulberry juice prevent obesity development in C57BL/6 Mice. PLoS ONE 2013, 8, e77585. [CrossRef] [PubMed]

13. Grace, M.H.; Ribnicky, D.M.; Kuhn, P.; Poulev, A.; Logendra, S.; Yousef, G.G.; Raskin, I.; Lila, M.A. Hypoglycemic activity of a novel anthocyanin-rich formulation from lowbush blueberry, Vaccinium angustifolium Aiton. Phytomedicine 2009, 16, 406-415. [CrossRef]

14. Huang, W.; Yao, L.; He, X.; Wang, L.; Li, M.; Yang, Y.; Wan, C. Hypoglycemic activity and constituents analysis of blueberry (Vaccinium corymbosum) fruit extracts. Diabetes Metab. Syndr. Obes. Targets Ther. 2018, 11, 357-366. [CrossRef] [PubMed]

15. Takikawa, M.; Inoue, S.; Horio, F.; Tsuda, T. Dietary anthocyanin-rich bilberry extract ameliorates hyperglycemia and insulin sensitivity via Activation of AMP-activated protein kinase in diabetic mice. J. Nutr. 2010, 140, 527-533. [CrossRef] [PubMed]

16. Nair, A.R.; Elks, C.M.; Vila, J.; Del Piero, F.; Paulsen, D.B.; Francis, J. A Blueberry-enriched diet improves renal function and reduces oxidative stress in metabolic syndrome animals: Potential mechanism of TLR4-MAPK signaling pathway. PLoS ONE 2014, 9, e111976. [CrossRef] [PubMed]

17. Nakajima, J.I.; Tanaka, I.; Seo, S.; Yamazaki, M.; Saito, K. LC/PDA/ESI-MS profiling and radical scavenging activity of anthocyanins in various berries. J. Biomed. Biotechnol. 2004, 2004, 241-247. [CrossRef]

18. Mykkänen, O.T.; Huotari, A.; Herzig, K.-H.; Dunlop, T.W.; Mykkänen, H.; Kirjavainen, P.V. Wild blueberries (Vaccinium myrtillus) alleviate inflammation and hypertension associated with developing obesity in mice fed with a high-fat Diet. PLoS ONE 2014, 9, e114790. [CrossRef]

19. Prior, R.L.; Wu, X.; Gu, L.; Hager, T.J.; Hager, A.; Howard, L.R. Whole berries versus berry anthocyanins: Interactions with dietary fat levels in the C57BL/6J mouse model of obesity. J. Agric. Food Chem. 2008, 56, 647-653. [CrossRef]

20. Basu, A.; Du, M.; Leyva, M.J.; Sanchez, K.; Betts, N.M.; Wu, M.; Aston, C.E.; Lyons, T.J. Blueberries decrease cardiovascular risk factors in obese men and women with metabolic syndrome. J. Nutr. 2010, 140, 1582-1587. [CrossRef]

21. Stull, A.J.; Cash, K.C.; Champagne, C.M.; Gupta, A.K.; Boston, R.C.; Beyl, R.A.; Johnson, W.D.; Cefalu, W.T. Blueberries improve endothelial function, but not blood pressure, in adults with metabolic syndrome: A randomized, double-blind, placebo-controlled clinical trial. Nutrients 2015, 7, 4107-4123. [CrossRef]

22. Johnson, M.H.; Wallig, M.; Luna-Vital, D.A.; De Mejia, E.G. Alcohol-free fermented blueberry-blackberry beverage phenolic extract attenuates diet-induced obesity and blood glucose in C57BL/6J mice. J. Nutr. Biochem. 2016, 31, 45-59. [CrossRef] [PubMed]

23. Vuong, T.; Benhaddou-Andaloussi, A.; Brault, A.; Harbilas, D.; Martineau, L.C.; Vallerand, D.; Ramassamy, C.; Matar, C.; Haddad, P.S. Antiobesity and antidiabetic effects of biotransformed blueberry juice in KKAy mice. Int. J. Obes. 2009, 33, 1166-1173. [CrossRef] [PubMed]

24. Bell, L.; Lamport, D.J.; Butler, L.T.; Williams, C. A study of glycaemic effects following acute anthocyanin-rich blueberry supplementation in healthy young adults. Food Funct. 2017, 8, 3104-3110. [CrossRef]

25. Vendrame, S.; Daugherty, A.; Kristo, A.S.; Riso, P.; Klimis-Zacas, D. Wild blueberry (Vaccinium angustifolium) consumption improves inflammatory status in the obese Zucker rat model of the metabolic syndrome. J. Nutr. Biochem. 2013, 24, 1508-1512. [CrossRef] [PubMed]

26. Zhu, J.; Liu, W.; Yu, J.; Zou, S.; Wang, J.; Yao, W.; Gao, X. Characterization and hypoglycemic effect of a polysaccharide extracted from the fruit of Lycium barbarum L. Carbohydr. Polym. 2013, 98, 8-16. [CrossRef]

27. Bharat, D.; Cavalcanti, R.R.M.; Petersen, C.; Begaye, N.; Cutler, B.R.; Costa, M.M.A.; Ramos, R.K.L.G.; Ferreira, M.R.; Li, Y.; Bharath, L.P.; et al. Blueberry metabolites attenuate lipotoxicity-induced endothelial dysfunction. Mol. Nutr. Food Res. 2017, 62, 1700601. [CrossRef]

28. Bloedon, T.; Vendrame, S.; Bolton, J.; Lehnhard, R.; Riso, P.; Klimis-Zacas, D. The effect of wild blueberry (Vaccinium angustifolium) consumption on oxidative stress, inflammation, and DNA damage associated with exercise. Comp. Exerc. Physiol. 2015, 11, 173-181. [CrossRef]

29. Li, F.; Zhang, B.; Chen, G.; Fu, X. The novel contributors of anti-diabetic potential in mulberry polyphenols revealed by UHPLC-HR-ESI-TOF-MS/MS. Food Res. Int. 2017, 100, 873-884. [CrossRef] 
30. Ren, G.; Rimando, A.M.; Mathews, S.T. AMPK activation by pterostilbene contributes to suppression of hepatic gluconeogenic gene expression and glucose production in H4IIE cells. Biochem. Biophys. Res. Commun. 2018, 498, 640-645. [CrossRef]

31. Lee, S.I.; Keirsey, K.; Kirkland, R.I.; Grunewald, Z.; Fischer, J.G.; De La Serre, C.B. Blueberry supplementation influences the gut microbiota, inflammation, and insulin resistance in high-fat-diet-fed rats. J. Nutr. 2018, 148, 209-219. [CrossRef]

32. Vendrame, S.; Guglielmetti, S.; Riso, P.; Arioli, S.; Klimis-Zacas, D.; Porrini, M. Six-week consumption of a wild blueberry powder drink increases bifidobacteria in the human gut. J. Agric. Food Chem. 2011, 59, 12815-12820. [CrossRef] [PubMed]

33. Polewski, M.A.; Esquivel-Alvarado, D.; Wedde, N.S.; Kruger, C.G.; Reed, J.D. Isolation and characterization of blueberry polyphenolic components and their effects on gut barrier dysfunction. J. Agric. Food Chem. 2019, 68, 2940-2947. [CrossRef] [PubMed]

34. Rodríguez-Daza, M.-C.; Daoust, L.; Boutkrabt, L.; Pilon, G.; Varin, T.; Dudonné, S.; Levy, É.; Marette, A.; Roy, D.; Desjardins, Y. Wild blueberry proanthocyanidins shape distinct gut microbiota profile and influence glucose homeostasis and intestinal phenotypes in high-fat high-sucrose fed mice. Sci. Rep. 2020, 10, 1-16. [CrossRef] [PubMed]

35. Yang, H.; Pang, W.; Lu, H.; Cheng, D.; Yan, X.; Cheng, Y.; Jiang, Y. Comparison of metabolic profiling of cyanidin-3-o-galactoside and extracts from blueberry in aged mice. J. Agric. Food Chem. 2011, 59, 2069-2076. [CrossRef]

36. Harris, C.; Burt, A.J.; Saleem, A.; Le, P.M.; Martineau, L.C.; Haddad, P.S.; Bennett, S.A.L.; Arnason, J.T. A single HPLC-PAD-APCI/MS method for the quantitative comparison of phenolic compounds found in leaf, stem, root and fruit extracts of Vaccinium angustifolium. Phytochem. Anal. 2007, 18, 161-169. [CrossRef]

37. Lätti, A.K.; Kainulainen, P.S.; Hayirlioglu-Ayaz, S.; Ayaz, F.A.; Riihinen, K.R. Characterization of anthocyanins in caucasian blueberries (Vaccinium arctostaphylos L.) native to Turkey. J. Agric. Food Chem. 2009, 57, 5244-5249. [CrossRef]

38. Može, S.; Polak, T.; Gašperlin, L.; Koron, D.; Vanzo, A.; Ulrih, N.P.; Abram, V. Phenolics in slovenian bilberries (Vaccinium myrtillus L.) and blueberries (Vaccinium corymbosum L.). J. Agric. Food Chem. 2011, 59, 6998-7004. [CrossRef]

39. Shi, M.; Loftus, H.; McAinch, A.J.; Su, X.Q. Blueberry as a source of bioactive compounds for the treatment of obesity, type 2 diabetes and chronic inflammation. J. Funct. Foods 2017, 30, 16-29. [CrossRef]

40. Seymour, E.M.; Tanone, I.I.; Urcuyo-Llanes, D.E.; Lewis, S.K.; Kirakosyan, A.; Kondoleon, M.G.; Kaufman, P.B.; Bolling, S.F. Blueberry intake alters skeletal muscle and adipose tissue peroxisome proliferator-activated receptor activity and reduces insulin resistance in obese rats. J. Med. Food 2011, 14, 1511-1518. [CrossRef]

41. Rebello, C.J.; Burton, J.; Heiman, M.; Greenway, F.L. Gastrointestinal microbiome modulator improves glucose tolerance in overweight and obese subjects: A randomized controlled pilot trial. J. Diabetes Complicat. 2015, 29, 1272-1276. [CrossRef]

42. Li, D.; Zhang, Y.; Liu, Y.; Sun, R.; Xia, M. Purified anthocyanin supplementation reduces dyslipidemia, enhances antioxidant capacity, and prevents insulin resistance in diabetic patients. J. Nutr. 2015, 145, 742-748. [CrossRef] [PubMed]

43. Asgary, S.; Rafieian-Kopaei, M.; Sahebkar, A.; Shamsi, F.; Goli-Malekabadi, N. Anti-hyperglycemic and anti-hyperlipidemic effects of Vaccinium myrtillusfruit in experimentally induced diabetes (antidiabetic effect of Vaccinium myrtillusfruit). J. Sci. Food Agric. 2015, 96, 764-768. [CrossRef] [PubMed]

44. Kim, J.; Kim, C.-S.; Lee, Y.M.; Sohn, E.; Jo, K.; Kim, J.S. Vaccinium myrtillus extract prevents or delays the onset of diabetes-Induced blood-retinal barrier breakdown. Int. J. Food Sci. Nutr. 2015, 66, 236-242. [CrossRef] [PubMed]

45. Bao, L.; Yao, X.S.; Yau, C.C.; Tsi, D.; Chia, C.-S.; Nagai, H.; Kurihara, H. Protective effects of bilberry (Vaccinium myrtillus L.) extract on restraint stress-induced liver damage in mice. J. Agric. Food Chem. 2008, 56, 7803-7807. [CrossRef]

46. Cohen-Boulakia, F.; Valensi, P.E.; Boulahdour, H.; Lestrade, R.; Dufour-Lamartinie, J.-F.; Hort-Legrand, C.; Behar, A. In vivo sequential study of skeletal muscle capillary permeability in diabetic rats: Effect of anthocyanosides. Metabolism 2000, 49, 880-885. [CrossRef] 
47. Ferreira, F.M.; Peixoto, F.; Nunes, E.; Sena, C.; Seiça, R.; Santos, M.S. MitoTeas: Vaccinium myrtillus and Geranium robertianum decoctions improve diabetic Goto-Kakizaki rats hepatic mitochondrial oxidative phosphorylation. Biochim. Biophys. Acta 2010, 1797, 79-80. [CrossRef]

48. Hoggard, N.; Cruickshank, M.; Moar, K.M.; Bestwick, C.; Holst, J.J.; Russell, W.R.; Horgan, G. A single supplement of a standardised bilberry (Vaccinium myrtillus L.) extract (36\% wet weight anthocyanins) modifies glycaemic response in individuals with type 2 diabetes controlled by diet and lifestyle. J. Nutr. Sci. 2013, 2, e22. [CrossRef]

49. Alnajjar, M.; Barik, S.K.; Bestwick, C.; Campbell, F.; Cruickshank, M.; Farquharson, F.; Holtrop, G.; Horgan, G.; Louis, P.; Moar, K.-M.; et al. Anthocyanin-enriched bilberry extract attenuates glycaemic response in overweight volunteers without changes in insulin. J. Funct. Foods 2020, 64, 103597. [CrossRef]

50. Granfeldt, Y.; Björck, I. A bilberry drink with fermented oatmeal decreases postprandial insulin demand in young healthy adults. Nutr. J. 2011, 10, 57. [CrossRef]

51. Karlsen, A.; Paur, I.; Bøhn, S.K.; Sakhi, A.K.; Borge, G.I.; Serafini, M.; Erlund, I.; Laake, P.; Tonstad, S.; Blomhoff, R. Bilberry juice modulates plasma concentration of NF- $\kappa B$ related inflammatory markers in subjects at increased risk of CVD. Eur. J. Nutr. 2010, 49, 345-355. [CrossRef]

52. Erlund, I.; Marniemi, J.; Hakala, P.; Alfthan, G.; Meririnne, E.; Aro, A. Consumption of black currants, lingonberries and bilberries increases serum quercetin concentrations. Eur. J. Clin. Nutr. 2003, 57, 37-42. [CrossRef] [PubMed]

53. Kolehmainen, M.; Mykkänen, O.; Kirjavainen, P.V.; Leppänen, T.; Moilanen, E.; Adriaens, M.E.; Laaksonen, D.E.; Hallikainen, M.; Puupponen-Pimia, R.; Pulkkinen, L.; et al. Bilberries reduce low-grade inflammation in individuals with features of metabolic syndrome. Mol. Nutr. Food Res. 2012, 56, 1501-1510. [CrossRef] [PubMed]

54. Anhê, F.F.; Roy, D.; Pilon, G.; Dudonné, S.; Matamoros, S.; Varin, T.V.; Garofalo, C.; Moine, Q.; Desjardins, Y.; Levy, E.; et al. A polyphenol-rich cranberry extract protects from diet-induced obesity, insulin resistance and intestinal inflammation in association with increasedAkkermansiaspp. population in the gut microbiota of mice. Gut 2014, 64, 872-883. [CrossRef] [PubMed]

55. Zhu, M.; Hu, J.; Perez, E.; Phillips, D.; Kim, W.; Ghaedian, R.; Napora, J.K.; Zou, S. Effects of long-term cranberry supplementation on endocrine pancreas in aging Rats. J. Gerontol. Ser. A Biol. Sci. Med. Sci. 2011, 66, 1139-1151. [CrossRef]

56. Schell, J.; Betts, N.M.; Foster, M.; Scofield, R.H.; Basu, A. Cranberries improve postprandial glucose excursions in type 2 diabetes. Food Funct. 2017, 8, 3083-3090. [CrossRef]

57. Lee, I.T.; Chan, Y.C.; Lin, C.W.; Lee, W.J.; Sheu, W.H.-H. Effect of cranberry extracts on lipid profiles in subjects with Type 2 diabetes. Diabet. Med. 2008, 25, 1473-1477. [CrossRef]

58. Wilson, T.; Meyers, S.; Singh, A.; Limburg, P.; Vorsa, N. Favorable glycemic response of Type 2 diabetics to low-calorie cranberry juice. J. Food Sci. 2008, 73, H241-H245. [CrossRef]

59. Wilson, T.; Singh, A.P.; Vorsa, N.; Goettl, C.D.; Kittleson, K.M.; Roe, C.M.; Kastello, G.M.; Ragsdale, F.R. Human glycemic response and phenolic content of unsweetened cranberry juice. J. Med. Food 2008, 11, 46-54. [CrossRef]

60. Wilson, T.; Luebke, J.L.; Morcomb, E.F.; Carrell, E.J.; Leveranz, M.C.; Kobs, L.; Schmidt, T.P.; Limburg, P.J.; Vorsa, N.; Singh, A.P. Glycemic responses to sweetened dried and raw cranberries in humans with Type 2 diabetes. J. Food Sci. 2010, 75, H218-H223. [CrossRef]

61. Duffey, K.J.; Sutherland, L.A. Adult consumers of cranberry juice cocktail have lower C-reactive protein levels compared with nonconsumers. Nutr. Res. 2015, 35, 118-126. [CrossRef]

62. Törrönen, R.; Sarkkinen, E.; Niskanen, T.; Tapola, N.; Kilpi, K.; Niskanen, L. Postprandial glucose, insulin and glucagon-like peptide 1 responses to sucrose ingested with berries in healthy subjects. Br. J. Nutr. 2011, 107, 1445-1451. [CrossRef] [PubMed]

63. Javid, A.Z.; Maghsoumi-Norouzabad, L.; Ashrafzadeh, E.; Yousefimanesh, H.A.; Zakerkish, M.; Angali, K.A.; Ravanbakhsh, M.; Babaei, H. Impact of cranberry juice enriched with Omega-3 Fatty acids adjunct with nonsurgical periodontal treatment on metabolic control and periodontal status in Type 2 patients with diabetes with periodontal Disease. J. Am. Coll. Nutr. 2017, 37, 71-79. [CrossRef] [PubMed]

64. Shidfar, F.; Heydari, I.; Hajimiresmaiel, S.J.; Hosseini, S.; Shidfar, S.; Amiri, F. The effects of cranberry juice on serum glucose, apoB, apoA-I, Lp (a), and Paraoxonase-1 activity in type 2 diabetic male patients. J. Res. Med. Sci. 2012, 17, 355-360. [PubMed] 
65. Novotny, A.; Baer, J.; Khoo, D.J.; Gebauer, C.; Charron, S.K. Cranberry juice consumption lowers markers of cardiometabolic risk, including blood pressure and circulating c-reactive protein, triglyceride, and glucose concentrations in adults. J. Nutr. 2015, 145, 1185-1193. [CrossRef] [PubMed]

66. Simão, T.N.C.; Lozovoy, M.A.B.; Simão, A.N.C.; Oliveira, S.R.; Venturini, D.; Morimoto, H.K.; Miglioranza, L.H.S.; Dichi, I. Reduced-energy cranberry juice increases folic acid and adiponectin and reduces homocysteine and oxidative stress in patients with the metabolic syndrome. Br. J. Nutr. 2013, 110, 1885-1894. [CrossRef]

67. Ruel, G.; Lapointe, A.; Pomerleau, S.; Couture, P.; Lemieux, S.; Lamarche, B.; Couillard, C. Evidence that cranberry juice may improve augmentation index in overweight men. Nutr. Res. 2013, 33, 41-49. [CrossRef]

68. Ruel, G.; Pomerleau, S.; Couture, P.; Lemieux, S.; Lamarche, B.; Couillard, C. Plasma matrix metalloproteinase (MMP)-9 levels are reduced following low-calorie cranberry juice supplementation in men. J. Am. Coll. Nutr. 2009, 28, 694-701. [CrossRef]

69. Ruel, G.; Pomerleau, S.; Couture, P.; Lemieux, S.; Lamarche, B.; Couillard, C. Low-calorie cranberry juice supplementation reduces plasma oxidized LDL and cell adhesion molecule concentrations in men. Br. J. Nutr. 2008, 99, 352-359. [CrossRef]

70. Ruel, G.; Pomerleau, S.; Couture, P.; Lemieux, S.; Lamarche, B.; Couillard, C. Favourable impact of low-calorie cranberry juice consumption on plasma HDL-cholesterol concentrations in men. Br. J. Nutr. 2006, 96, 357-364. [CrossRef]

71. Ruel, G.; Pomerleau, S.; Couture, P.; Lamarche, B.; Couillard, C. Changes in plasma antioxidant capacity and oxidized low-density lipoprotein levels in men after short-term cranberry juice consumption. Metabolism 2005, 54, 856-861. [CrossRef]

72. Suh, J.H.; Romain, C.; González-Barrio, R.; Cristol, J.P.; Teissedre, P.L.; Crozier, A.; Rouanet, J.M. Raspberry juice consumption, oxidative stress and reduction of atherosclerosis risk factors in hypercholesterolemic golden Syrian hamsters. Food Funct. 2011, 2, 400-405. [CrossRef] [PubMed]

73. SanGiovanni, E.; Vrhovšek, U.; Rossoni, G.; Colombo, E.; Brunelli, C.; Brembati, L.; Trivulzio, S.; Gasperotti, M.; Mattivi, F.; Bosisio, E.; et al. Ellagitannins from rubus berries for the control of gastric inflammation: In vitro and in vivo studies. PLOS ONE 2013, 8, e71762. [CrossRef]

74. Jean-Gilles, D.; Li, L.; Ma, H.; Yuan, T.; Chichester, I.C.O.; Seeram, N.P. Anti-inflammatory effects of polyphenolic-enriched red raspberry extract in an antigen-induced arthritis rat model. J. Agric. Food Chem. 2011, 60, 5755-5762. [CrossRef] [PubMed]

75. Gião, M.; Pestana, D.; Faria, A.; Guimarães, J.T.; Pintado, M.; Calhau, C.; Azevedo, I.; Malcata, F.X. Effects of extracts of selected medicinal plants upon hepatic oxidative stress. J. Med. Food 2010, 13, 131-136. [CrossRef] [PubMed]

76. Noratto, G.; Chew, B.P.; Atienza, L.M. Red raspberry (Rubus idaeus L.) intake decreases oxidative stress in obese diabetic (db/db) mice. Food Chem. 2017, 227, 305-314. [CrossRef] [PubMed]

77. Kirakosyan, A.; Seymour, E.M.; Kondoleon, N.; Gutiérrez, E.; Wolforth, J.; Bolling, S. The intake of red raspberry fruit is inversely related to cardiac risk factors associated with metabolic syndrome. J. Funct. Foods 2018, 41, 83-89. [CrossRef]

78. Jakobsdottir, G.; Blanco, N.; Xu, J.; Ahrné, S.; Molin, G.; Sterner, O.; Nyman, M. Formation of short-chain fatty acids, excretion of anthocyanins, and microbial diversity in rats fed blackcurrants, blackberries, and raspberries. J. Nutr. Metab. 2013, 2013, 1-12. [CrossRef] [PubMed]

79. Pan, P.; Lam, V.; Salzman, N.H.; Huang, Y.W.; Yu, J.; Zhang, J.; Wang, L. Black raspberries and their anthocyanin and fiber fractions alter the composition and diversity of gut microbiota in F-344 rats. Nutr. Cancer 2017, 69, 943-951. [CrossRef] [PubMed]

80. Su, H.; Xie, L.; Xu, Y.; Ke, H.; Bao, T.; Li, Y.; Chen, W. Pelargonidin-3-O-glucoside derived from wild raspberry exerts antihyperglycemic effect by inducing autophagy and modulating gut microbiota. J. Agric. Food Chem. 2019. [CrossRef]

81. Tu, P.; Bian, X.; Chi, L.; Gao, B.; Ru, H.; Knobloch, T.J.; Weghorst, C.M.; Lu, K. Characterization of the functional changes in mouse gut microbiome associated with increased akkermansia muciniphila population modulated by dietary black raspberries. ACS Omega 2018, 3, 10927-10937. [CrossRef]

82. Atienza, L.E.; Garcia, P.; Noratto, G. Effects of raspberry on biomarkers of diabetes, cardiovascular disease (cvd) and oxidative stress in obese diabetic (db/db) mice. FASEB J. 2015, 29, LB283. 
83. Luo, T.; Miranda-Garcia, O.; Adamson, A.; Sasaki, G.; Shay, N. Development of obesity is reduced in high-fat fed mice fed whole raspberries, raspberry juice concentrate, and a combination of the raspberry phytochemicals ellagic acid and raspberry ketone. J. Berry Res. 2016, 6, 213-223. [CrossRef]

84. Guo, H.; Xia, M.; Zou, T.; Ling, W.; Zhong, R.; Zhang, W. Cyanidin 3-glucoside attenuates obesity-associated insulin resistance and hepatic steatosis in high-fat diet-fed and db/db mice via the transcription factor FoxO1. J. Nutr. Biochem. 2012, 23, 349-360. [CrossRef]

85. Iino, T.; Tashima, K.; Umeda, M.; Ogawa, Y.; Takeeda, M.; Takata, K.; Takeuchi, K. Effect of ellagic acid on gastric damage induced in ischemic rat stomachs following ammonia or reperfusion. Life Sci. 2002, 70, 1139-1150. [CrossRef]

86. Rosillo, M.A. Protective effect of ellagic acid, a natural polyphenolic compound, in a murine model of Crohn's disease. Biochem. Pharmacol. 2011, 82, 737-745. [CrossRef] [PubMed]

87. Zhao, L.; Zou, T.; Gomez, N.A.; Wang, B.; Zhu, M.-J.; Du, M. Raspberry alleviates obesity-induced inflammation and insulin resistance in skeletal muscle through activation of AMP-activated protein kinase (AMPK) $\alpha 1$. Nutr. Diabetes 2018, 8, 39. [CrossRef] [PubMed]

88. Zhu, M.; Kang, Y.; Xue, Y.; Liang, X.; García, M.P.G.; Rodgers, D.; Kagel, D.R.; Du, M. Red raspberries suppress NLRP3 inflammasome and attenuate metabolic abnormalities in diet-induced obese mice. J. Nutr. Biochem. 2017, 53, 96-103. [CrossRef]

89. Sasaki, R.; Nishimura, N.; Hoshino, H.; Isa, Y.; Kadowaki, M.; Ichi, T.; Tanaka, A.; Nishiumi, S.; Fukuda, I.; Ashida, H.; et al. Cyanidin 3-glucoside ameliorates hyperglycemia and insulin sensitivity due to downregulation of retinol binding protein 4 expression in diabetic mice. Biochem. Pharmacol. 2007, 74, 1619-1627. [CrossRef]

90. Kang, I.; Espín, J.C.; Carr, T.P.; Tomás-Barberán, F.A.; Chung, S. Raspberry seed flour attenuates high-sucrose diet-mediated hepatic stress and adipose tissue inflammation. J. Nutr. Biochem. 2016, 32, 64-72. [CrossRef]

91. Smith, T.J.; Karl, J.P.; Wilson, M.A.; Whitney, C.C.; Barrett, A.; Farhadi, N.F.; Chen, C.Y.O.; Montain, S.J. Glycaemic regulation, appetite and ex vivo oxidative stress in young adults following consumption of high-carbohydrate cereal bars fortified with polyphenol-rich berries. Br. J. Nutr. 2019, 121, 1026-1038. [CrossRef]

92. Clegg, M.E.; Pratt, M.; Meade, C.M.; Henry, C.J. The addition of raspberries and blueberries to a starch-based food does not alter the glycaemic response. Br. J. Nutr. 2011, 106, 335-338. [CrossRef] [PubMed]

93. Törrönen, R.; Kolehmainen, M.; Sarkkinen, E.; Poutanen, K.; Mykkänen, H.; Niskanen, L. Berries reduce postprandial insulin responses to wheat and rye breads in healthy women. J. Nutr. 2013, 143, 430-436. [CrossRef] [PubMed]

94. Schell, J.; Betts, N.M.; Lyons, T.J.; Basu, A. Raspberries improve postprandial glucose and acute and chronic inflammation in adults with Type 2 Diabetes. Ann. Nutr. Metab. 2019, 74, 165-174. [CrossRef] [PubMed]

95. Wu, T.; Yin, J.; Zhang, G.; Long, H.; Zheng, X. Mulberry and cherry anthocyanin consumption prevents oxidative stress and inflammation in diet-induced obese mice. Mol. Nutr. Food Res. 2016, 60, 687-694. [CrossRef] [PubMed]

96. Yan, F.; Zheng, X. Anthocyanin-rich mulberry fruit improves insulin resistance and protects hepatocytes against oxidative stress during hyperglycemia by regulating AMPK/ACC/mTOR pathway. J. Funct. Foods 2017, 30, 270-281. [CrossRef]

97. Srikanta, A.H.; Kumar, A.; Sukhdeo, S.V.; Peddha, M.S.; Govindaswamy, V. The antioxidant effect of mulberry and jamun fruit wines by ameliorating oxidative stress in streptozotocin-induced diabetic Wistar rats. Food Funct. 2016, 7, 4422-4431. [CrossRef]

98. Ha, U.S.; Bae, W.J.; Kim, S.J.; Yoon, B.I.; Jang, H.; Hong, S.H.; Lee, J.Y.; Hwang, S.Y.; Kim, S.W. Protective effect of cyanidin-3-O- $\beta$-D-glucopyranoside fraction from mulberry fruit pigment against oxidative damage in streptozotocin-induced diabetic rat bladder. Neurourol. Urodyn. 2012, 32, 493-499. [CrossRef]

99. Ren, C.; Zhang, Y.; Cui, W.; Lu, G.; Wang, Y.; Gao, H.; Huang, L.; Mu, Z. A polysaccharide extract of mulberry leaf ameliorates hepatic glucose metabolism and insulin signaling in rats with type 2 diabetes induced by high fat-diet and streptozotocin. Int. J. Biol. Macromol. 2015, 72, 951-959. [CrossRef]

100. Liu, L.K.; Chou, F.-P.; Chen, Y.C.; Chyau, C.C.; Ho, H.H.; Wang, C.J. Effects of mulberry (Morus albal.) extracts on lipid homeostasis in vitro and in vivo. J. Agric. Food Chem. 2009, 57, 7605-7611. [CrossRef] 
101. Wu, T.; Qi, X.; Liu, Y.; Guo, J.; Zhu, R.; Chen, W.; Zheng, X.; Yu, T. Dietary supplementation with purified mulberry (Morus australis Poir) anthocyanins suppresses body weight gain in high-fat diet fed C57BL/6 mice. Food Chem. 2013, 141, 482-487. [CrossRef]

102. Du, Q.; Zheng, J.; Xu, Y. Composition of anthocyanins in mulberry and their antioxidant activity. J. Food Compos. Anal. 2008, 21, 390-395. [CrossRef]

103. Peng, C.H.; Liu, L.K.; Chyau, C.C.; Wang, C.J.; Chuang, C.M.; Huang, C.N. Mulberry water extracts possess an anti-obesity effect and ability to inhibit hepatic lipogenesis and promote lipolysis. J. Agric. Food Chem. 2011, 59, 2663-2671. [CrossRef] [PubMed]

104. Tang, C.C.; Huang, H.P.; Lee, Y.J.; Tang, Y.H.; Wang, C.J. Hepatoprotective effect of mulberry water extracts on ethanol-induced liver injury via anti-inflammation and inhibition of lipogenesis in C57BL/6J mice. Food Chem. Toxicol. 2013, 62, 786-796. [CrossRef] [PubMed]

105. Yang, D.K.; Joo-Yong, L. Mulberry fruit extract ameliorates nonalcoholic fatty liver disease (NAFLD) through inhibition of mitochondrial oxidative stress in rats. Evid. Based Complement. Altern. Med. 2018, 2018, 8165716. [CrossRef] [PubMed]

106. Wattanathorn, J.; Kawvised, S.; Thukham-Mee, W. Encapsulated Mulberry Fruit Extract Alleviates Changes in an Animal Model of Menopause with Metabolic Syndrome. Oxidative Med. Cell. Longev. 2019, 2019, 5360560. [CrossRef]

107. Sheng, Y.; Liu, J.; Zheng, S.; Liang, F.; Luo, Y.; Huang, K.; Xu, W.; He, X. Mulberry leaves ameliorate obesity through enhancing brown adipose tissue activity and modulating gut microbiota. Food Funct. 2019, 10, 4771-4781. [CrossRef] [PubMed]

108. Chen, C.; You, L.; Huang, Q.; Fu, X.; Zhang, B.; Liu, R.-H.; Li, C. Modulation of gut microbiota by mulberry fruit polysaccharide treatment of obese diabetic db/db mice. Food Funct. 2018, 9, 3732-3742. [CrossRef]

109. Pierre, W. Hypoglycemic and hypolipidemic effects of Bersama engleriana leaves in nicotinamide/streptozotocin-induced type 2 diabetic rats. BMC Complement. Altern. Med. 2012, 12, 264. [CrossRef]

110. Jiao, Y.; Wang, X.; Jiang, X.; Kong, F.; Wang, S.; Yan, C. Antidiabetic effects of Morus alba fruit polysaccharides on high-fat diet- and streptozotocin-induced type 2 diabetes in rats. J. Ethnopharmacol. 2017, 199, 119-127. [CrossRef]

111. Eid, H.M.; Ouchfoun, M.; Brault, A.; Vallerand, D.; Musallam, L.; Arnason, J.T.; Haddad, P.S. Lingonberry (Vaccinium vitis-idaea L.) exhibits antidiabetic activities in a mouse model of diet-induced obesity. Evid. Based Complement. Altern. Med. 2014, 2014,1-10. [CrossRef]

112. Kivimäki, A.S.; Ehlers, P.I.; Siltari, A.; Turpeinen, A.M.; Vapaatalo, H.; Korpela, R. Lingonberry, cranberry and blackcurrant juices affect mRNA expressions of inflammatory and atherothrombotic markers of SHR in a long-term treatment. J. Funct. Foods 2012, 4, 496-503. [CrossRef]

113. Heyman-Lindén, L.; Axling, U.; Blanco, N.; Sterner, O.; Holm, C.; Berger, K. Evaluation of beneficial metabolic effects of berries in high-fat fed C57BL/6J Mice. J. Nutr. Metab. 2014, 2014, 1-12. [CrossRef] [PubMed]

114. Heyman-Lindén, L.; Kotowska, D.; Sand, E.; Bjursell, M.; Plaza, M.; Turner, C.; Holm, C.; Fåk, F.; Berger, K. Lingonberries alter the gut microbiota and prevent low-grade inflammation in high-fat diet fed mice. Food Nutr. Res. 2016, 60, 29993. [CrossRef] [PubMed]

115. Matziouridou, C.; Marungruang, N.; Nguyen, T.D.; Nyman, M.; Fak, F. Lingonberries reduce atherosclerosis inApoe-/-mice in association with altered gut microbiota composition and improved lipid profile. Mol. Nutr. Food Res. 2016, 60, 1150-1160. [CrossRef]

116. Al-Hamimi, S.; Heyman-Lindén, L.; Plaza, M.; Turner, C.; Berger, K.; Spégel, P. Alterations in the plasma metabolite profile associated with improved hepatic function and glycemia in mice fed lingonberry supplemented high-fat diets. Mol. Nutr. Food Res. 2016, 61, 1600442. [CrossRef]

117. Heyman-Lindén, L.; Seki, Y.; Storm, P.; Jones, H.A.; Charron, M.J.; Berger, K.; Holm, C. Berry intake changes hepatic gene expression and DNA methylation patterns associated with high-fat diet. J. Nutr. Biochem. 2016, 27, 79-95. [CrossRef]

118. Marungruang, N.; Kovalenko, T.; Osadchenko, I.; Voss, U.; Huang, F.; Burleigh, S.; Ushakova, A.A.; Skibo, G.; Nyman, M.; Prykhod'Ko, O.; et al. Lingonberries and their two separated fractions differently alter the gut microbiota, improve metabolic functions, reduce gut inflammatory properties, and improve brain function in ApoE-/- mice fed high-fat diet. Nutr. Neurosci. 2018, 23, 1-13. [CrossRef] 
119. Huang, F.; Nilholm, C.; Roth, B.; Linninge, C.; Hoglund, P.; Nyman, M.; Ohlsson, B. Anthropometric and metabolic improvements in human type 2 diabetes after introduction of an Okinawan-based Nordic diet are not associated with changes in microbial diversity or SCFA concentrations. Int. J. Food Sci. Nutr. 2017, 69, 729-740. [CrossRef]

120. Linderborg, K.M.; Järvinen, R.; Lehtonen, H.M.; Viitanen, M.; Kallio, H. The fiber and/or polyphenols present in lingonberries null the glycemic effect of the sugars present in the berries when consumed together with added glucose in healthy human volunteers. Nutr. Res. 2012, 32, 471-478. [CrossRef]

121. Furlan, C.; Valle, S.C.; Maróstica, M.R.; Östman, E.; Björck, I.; Tovar, J. Effect of bilberries, lingonberries and cinnamon on cardiometabolic risk-associated markers following a hypercaloric-hyperlipidic breakfast. J. Funct. Foods 2019, 60, 103443. [CrossRef]

122. Rokka, S.; Ketoja, E.; Järvenpää, E.; Tahvonen, R. The glycaemic and C-peptide responses of foods rich in dietary fibre from oat, buckwheat and lingonberry. Int. J. Food Sci. Nutr. 2013, 64, 528-534. [CrossRef] [PubMed]

123. Törrönen, R.; Kolehmainen, M.; Sarkkinen, E.; Mykkänen, H.; Niskanen, L. Postprandial glucose, insulin, and free fatty acid responses to sucrose consumed with blackcurrants and lingonberries in healthy women. Am. J. Clin. Nutr. 2012, 96, 527-533. [CrossRef] [PubMed]

124. Lehtonen, H.-M.; Lindstedt, A.; Järvinen, R.; Sinkkonen, J.; Graça, G.; Viitanen, M.; Kallio, H.; Gil, A.M. 1H NMR-based metabolic fingerprinting of urine metabolites after consumption of lingonberries (Vaccinium vitis-idaea) with a high-fat meal. Food Chem. 2013, 138, 982-990. [CrossRef] [PubMed]

125. Hassimotto, N.M.A.; Lajolo, F.M. Antioxidant status in rats after long-term intake of anthocyanins and ellagitannins from blackberries. J. Sci. Food Agric. 2010, 91, 523-531. [CrossRef]

126. Wu, T.; Gao, Y.; Guo, X.; Zhang, M.; Gong, L. Blackberry and blueberry anthocyanin supplementation counteract high-fat-diet-induced obesity by alleviating oxidative stress and inflammation and accelerating energy expenditure. Oxidative Med. Cell. Longev. 2018, 2018, 1-9. [CrossRef]

127. Kim, B.; Gil Lee, S.; Park, Y.-K.; Ku, C.S.; Pham, T.X.; Wegner, C.J.; Yang, Y.; Koo, S.I.; Chun, O.K.; Lee, J.Y. Blueberry, blackberry, and blackcurrant differentially affect plasma lipids and pro-inflammatory markers in diet-induced obesity mice. Nutr. Res. Pract. 2016, 10, 494-500. [CrossRef]

128. Meireles, M.; Rodríguez-Alcalá, L.M.; Marques, C.; Norberto, S.; Freitas, J.; Fernandes, I.; Mateus, N.; Gomes, A.; Faria, A.; Calhau, C. Effect of chronic consumption of blackberry extract on high-fat induced obesity in rats and its correlation with metabolic and brain outcomes. Food Funct. 2016, 7, 127-139. [CrossRef]

129. Azofeifa, G.; Quesada, S.; Navarro, L.; Hidalgo, O.; Portet, K.; Pérez, A.M.; Vaillant, F.; Poucheret, P.; Michel, A. Hypoglycaemic, hypolipidaemic and antioxidant effects of blackberry beverage consumption in streptozotocin-induced diabetic rats. J. Funct. Foods 2016, 26, 330-337. [CrossRef]

130. Kosmala, M.; Jurgoński, A.; Juskiewicz, J.; Karlińska, E.; Macierzynski, J.; Rój, E.; Zduńczyk, Z. Chemical composition of blackberry press cake, polyphenolic extract, and defatted seeds, and their effects on cecal fermentation, bacterial metabolites, and blood lipid profile in rats. J. Agric. Food Chem. 2017, 65, 5470-5479. [CrossRef]

131. Salyer, J.; Park, S.H.; Ricke, S.C.; Lee, S.O. Analysis of microbial populations and metabolism of anthocyanins by mice gut microflora fed with blackberry powder. J. Nutr. Food Sci. 2013, 3. [CrossRef]

132. Jenkins, D.J.A.; Srichaikul, K.; Kendall, C.W.C.; Sievenpiper, J.L.; Abdulnour, S.; Mirrahimi, A.; Meneses, C.; Nishi, S.; He, X.; Lee, S.; et al. The relation of low glycaemic index fruit consumption to glycaemic control and risk factors for coronary heart disease in type 2 diabetes. Diabetologia 2010, 54, 271-279. [CrossRef]

133. Hassimotto, N.M.A.; Pinto, M.D.S.; Lajolo, F.M. Antioxidant status in humans after consumption of blackberry (Rubus fruticosusL.) juices with and without defatted milk. J. Agric. Food Chem. 2008, 56, 11727-11733. [CrossRef] [PubMed]

134. Vafa, M.; Aghababaee, S.K.; Shidfar, F.; Tahavorgar, A.; Gohari, M.; Katebi, D.; Mohammadi, V. Effects of blackberry (Morus nigra L.) consumption on serum concentration of lipoproteins, apo A-I, apo B, and high-sensitivity-C-reactive protein and blood pressure in dyslipidemic patients. J. Res. Med. Sci. 2015, 20, 684-691. [CrossRef] [PubMed]

135. Mandave, P.C.; Khadke, S.; Karandikar, M.; Pandit, V.; Ranjekar, P.; Kuvalekar, A.; Mantri, N. Antidiabetic, lipid normalizing, and nephroprotective actions of the strawberry: A potent supplementary fruit. Int. J. Mol. Sci. 2017, 18, 124. [CrossRef] [PubMed] 
136. Oviedo-Solís, C.I.; Sandoval-Salazar, C.; Lozoya, E.; Maldonado-Aguilera, G.A.; Aguilar-Zavala, H.; Beltrán-Campos, V.; Pérez-Vázquez, V.; Ramírezemiliano, J. Ultraviolet light-C increases antioxidant capacity of the strawberry (Fragaria $x$ ananassa) in vitro and in high-fat diet-induced obese rats. Food Sci. Nutr. 2017, 5, 1004-1014. [CrossRef]

137. Parelman, M.A.; Storms, D.H.; Kirschke, C.P.; Huang, L.; Zunino, S.J. Dietary strawberry powder reduces blood glucose concentrations in obese and lean C57BL/6 mice, and selectively lowers plasma C-reactive protein in lean mice. Br. J. Nutr. 2012, 108, 1789-1799. [CrossRef]

138. Aranaz, P.; Romo-Hualde, A.; Zabala, M.; Navarro-Herrera, D.; De Galarreta, M.R.; Gil, A.G.; Martínez, J.A.; Aranaz, P.; Gonzalez, C.J. Freeze-dried strawberry and blueberry attenuates diet-induced obesity and insulin resistance in rats by inhibiting adipogenesis and lipogenesis. Food Funct. 2017, 8, 3999-4013. [CrossRef]

139. Diamanti, J.; Mezzetti, B.; Giampieri, F.; Alvarez-Suarez, J.M.; Quiles, J.L.; Gonzalez-Alonso, A.; Ramirez-Tortosa, M.D.C.; Granados-Principal, S.; Gonzáles-Paramás, A.M.; Santos-Buelga, C.; et al. Doxorubicin-induced oxidative stress in rats is efficiently counteracted by dietary anthocyanin differently enriched strawberry (Fragaria×ananassaDuch.). J. Agric. Food Chem. 2014, 62, 3935-3943. [CrossRef]

140. Sandoval-Salazar, C.; Oviedo-Solís, C.I.; Lozoya, E.; Aguilar-Zavala, H.; Solís-Ortiz, S.; Pérez-Vázquez, V.; Balcón-Pacheco, C.D.; Ramírezemiliano, J. Strawberry intake ameliorates oxidative stress and decreases GABA levels induced by high-fat diet in frontal cortex of rats. Antioxidants 2019, 8, 70. [CrossRef]

141. Petersen, C.; Wankhade, U.D.; Bharat, D.; Wong, K.; Mueller, J.E.; Chintapalli, S.V.; Piccolo, B.D.; Jalili, T.; Jia, Z.; Symons, J.D.; et al. Dietary supplementation with strawberry induces marked changes in the composition and functional potential of the gut microbiome in diabetic mice. J. Nutr. Biochem. 2019, 66, 63-69. [CrossRef]

142. Han, Y.; Song, M.; Gu, M.; Ren, D.; Zhu, X.; Cao, X.; Li, F.; Wang, W.; Cai, X.; Yuan, B.; et al. Dietary intake of whole strawberry inhibited colonic inflammation in dextran-sulfate-sodium-treated mice via restoring immune homeostasis and alleviating gut microbiota dysbiosis. J. Agric. Food Chem. 2019, 67, 9168-9177. [CrossRef]

143. Paquette, M.; Larqué, A.S.M.; Weisnagel, S.J.; Desjardins, Y.; Marois, J.; Pilon, G.; Dudonné, S.; Marette, A.; Jacques, H. Strawberry and cranberry polyphenols improve insulin sensitivity in insulin-resistant, non-diabetic adults: A parallel, double-blind, controlled and randomised clinical trial. Br. J. Nutr. 2017, 117, 519-531. [CrossRef] [PubMed]

144. Burton-Freeman, B.; Linares, A.; Hyson, D.; Kappagoda, T. Strawberry modulates LDL oxidation and postprandial lipemia in response to high-fat meal in overweight hyperlipidemic men and women. J. Am. Coll. Nutr. 2010, 29, 46-54. [CrossRef] [PubMed]

145. Kurotobi, T.; Fukuhara, K.; Inage, H.; Kimura, S. Glycemic index and postprandial blood glucose response to Japanese strawberry jam in normal adults. J. Nutr. Sci. Vitaminol. 2010, 56, 198-202. [CrossRef] [PubMed]

146. Ibero-Baraibar, I.; Cuervo, M.; Navas-Carretero, S.; Abete, I.; Zulet, M.A.; Martínez, J.A. Different postprandial acute response in healthy subjects to three strawberry jams varying in carbohydrate and antioxidant content: A randomized, crossover trial. Eur. J. Nutr. 2013, 53, 201-210. [CrossRef] [PubMed]

147. Amani, R.; Moazen, S.; Shahbazian, H.; Ahmadi, K.; Jalali, M.T. Flavonoid-rich beverage effects on lipid profile and blood pressure in diabetic patients. World J. Diabetes 2014, 5, 962-968. [CrossRef]

148. Ellis, C.L.; Edirisinghe, I.; Kappagoda, T.; Burton-Freeman, B. Attenuation of meal-induced inflammatory and thrombotic responses in overweight men and women after 6-week daily strawberry (Fragaria) intake. J. Atheroscler. Thromb. 2011, 18, 318-327. [CrossRef]

149. Edirisinghe, I.; Banaszewski, K.; Cappozzo, J.; Sandhya, K.; Ellis, C.L.; Tadapaneni, R.K.; Kappagoda, C.T.; Burton-Freeman, B.M. Strawberry anthocyanin and its association with postprandial inflammation and insulin. Br. J. Nutr. 2011, 106, 913-922. [CrossRef]

150. Park, E.; Edirisinghe, I.; Wei, H.; Vijayakumar, L.P.; Banaszewski, K.; Cappozzo, J.C.; Burton-Freeman, B. A dose-response evaluation of freeze-dried strawberries independent of fiber content on metabolic indices in abdominally obese individuals with insulin resistance in a randomized, single-blinded, diet-controlled crossover trial. Mol. Nutr. Food Res. 2016, 60, 1099-1109. [CrossRef]

151. Ivey, K.L.; Chan, A.T.; Izard, J.; Cassidy, A.; Rogers, G.B.; Rimm, E.B. Role of dietary flavonoid compounds in driving patterns of microbial community assembly. mBio 2019, 10, e01205-19. [CrossRef] 
152. Basu, A.; Morris, S.; Nguyen, A.; Betts, N.M.; Fu, D.; Lyons, T.J. Effects of dietary strawberry supplementation on antioxidant biomarkers in obese adults with above optimal serum lipids. J. Nutr. Metab. 2016, 2016, 1-9. [CrossRef] [PubMed]

153. Moazen, S.; Amani, R.; Rad, A.H.; Ahmadi, K.; Jalali, M.-T.; Shahbazian, H. Effects of freeze-dried strawberry supplementation on metabolic biomarkers of atherosclerosis in subjects with Type 2 Diabetes: A randomized double-blind controlled trial. Ann. Nutr. Metab. 2013, 63, 256-264. [CrossRef] [PubMed]

154. Luo, Q.; Cai, Y.; Yan, J.; Sun, M.; Corke, H. Hypoglycemic and hypolipidemic effects and antioxidant activity of fruit extracts from Lycium barbarum. Life Sci. 2004, 76, 137-149. [CrossRef]

155. Cui, B.K.; Liu, S.; Lin, X.; Wang, J.; Li, S.H.; Wang, Q.-B.; Li, S. Effects of Lycium barbarum aqueous and ethanol extracts on high-fat-diet induced oxidative stress in rat liver tissue. Molecules 2011, 16, 9116-9128. [CrossRef] [PubMed]

156. Jing, L.; Cui, G.; Feng, Q.; Xiao, Y. Evaluation of hypoglycemic activity of the polysaccharides extracted from Lycium Barbarum. Afr. J. Tradit. Complement. Altern. Med. 2009, 6, 579-584. [CrossRef] [PubMed]

157. Yin, J.; Wu, T. Anthocyanins from black wolfberry (Lycium ruthenicum Murr.) prevent inflammation and increase fecal fatty acid in diet-induced obese rats. RSC Adv. 2017, 7, 47848-47853. [CrossRef]

158. Zhao, R.; Li, Q.W.; Li, J.; Zhang, T. Protective effect of Lycium barbarum polysaccharide 4 on kidneys in streptozotocin-induced diabetic rats. Can. J. Physiol. Pharmacol. 2009, 87, 711-719. [CrossRef]

159. Hu, C.; Lee, Y.J.; Colitz, C.M.; Chang, C.J.; Lin, C.T. The protective effects of Lycium barbarum and Chrysanthemum morifolum on diabetic retinopathies in rats. Veter. Ophthalmol. 2012, 15, 65-71. [CrossRef]

160. Zhao, R.; Gao, X.; Zhang, T.; Li, X. Effects of Lycium barbarum. polysaccharide on type 2 diabetes mellitus rats by regulating biological rhythms. Iran. J. Basic Med. Sci 2016, 19, 1024-1030.

161. Al-Fartosy, A.J.M. Protective effect of galactomannan extracted from Iraqi Lycium Barbarum L. fruits against alloxan-induced diabetes in rats. Am. J. Biochem. Biotechnol. 2015, 11, 73-83. [CrossRef]

162. Du, M.; Hu, X.; Kou, L.; Zhang, B.; Zhang, C. Lycium barbarum Polysaccharide mediated the antidiabetic and antinephritic effects in diet-streptozotocin-induced diabetic sprague dawley rats via regulation of NF- $\mathrm{kB}$. BioMed Res. Int. 2016, 2016, 1-9. [CrossRef]

163. Ni, T.; Wei, G.; Yin, X.; Liu, X.; Liu, D. Original article Neuroprotective effect of Lycium barbarum on retina of Royal College of Surgeons (RCS) rats: A preliminary study. Folia Neuropathol. 2013, 2, 158-163. [CrossRef] [PubMed]

164. Kang, Y.; Yang, G.; Zhang, S.; Ross, C.F.; Zhu, M. Goji berry modulates gut microbiota and alleviates colitis in IL-10-deficient mice. Mol. Nutr. Food Res. 2018, 62, 1800535. [CrossRef] [PubMed]

165. Zhu, W.; Zhou, S.; Liu, J.; McLean, R.J.; Chu, W. Prebiotic, immuno-stimulating and gut microbiota-modulating effects of Lycium barbarum polysaccharide. Biomed. Pharmacother. 2020, 121, 109591. [CrossRef]

166. Amagase, H.; Sun, B.; Borek, C. Lycium barbarum (goji) juice improves in vivo antioxidant biomarkers in serum of healthy adults. Nutr. Res. 2009, 29, 19-25. [CrossRef]

167. Zanchet, M.Z.D.S.; Nardi, G.M.; Bratti, L.D.O.S.; Filippin, F.B.; Locatelli, C. Lycium Barbarum reduces abdominal fat and improves lipid profile and antioxidant status in patients with metabolic syndrome. Oxidative Med. Cell. Longev. 2017, 2017, 1-12. [CrossRef]

168. Xiao, J.; Liong, E.C.; Ching, Y.P.; Chang, R.C.C.; So, K.F.; Fung, M.L.; Tipoe, G.L. Lycium barbarum polysaccharides protect mice liver from carbon tetrachloride-induced oxidative stress and necroinflammation. J. Ethnopharmacol. 2012, 139, 462-470. [CrossRef]

169. Driessche, J.J.V.D.; Plat, J.; Plasqui, G.; Mensink, R.P. A single dose of goji berries does not affect postprandial energy expenditure and substrate oxidation in healthy, overweight men. J. Nutr. Metab. 2019, 2019, 1-6. [CrossRef]

170. De Oliveira, P.R.B.; Da Costa, C.A.; De Bem, G.F.; Cordeiro, V.S.C.; Santos, I.B.; De Carvalho, L.C.R.M.; Conceição, E.P.; Lisboa, P.C.; Ognibene, D.T.; Sousa, P.J.C.; et al. Euterpe oleracea mart.-derived polyphenols protect mice from diet-induced obesity and fatty liver by regulating hepatic lipogenesis and cholesterol Excretion. PLoS ONE 2015, 10, e0143721. [CrossRef]

171. Kim, J.Y.; Hong, J.-H.; Jung, H.K.; Jeong, Y.S.; Cho, K.H. Grape skin and loquat leaf extracts and acai puree have potent anti-atherosclerotic and anti-diabetic activity in vitro and in vivo in hypercholesterolemic zebrafish. Int. J. Mol. Med. 2012, 30, 606-614. [CrossRef] 
172. Sun, X.; Seeberger, J.; Alberico, T.; Wang, C.; Wheeler, C.T.; Schauss, A.G.; Zou, S. Açai palm fruit (Euterpe oleracea Mart.) pulp improves survival of flies on a high fat diet. Exp. Gerontol. 2010, 45, 243-251. [CrossRef] [PubMed]

173. De Castro, C.A.; Natali, A.J.; Cardoso, L.M.; Ferreira-Machado, A.B.; Novello, A.A.; Da Silva, K.A.; Tafuri, N.F.; Da Matta, S.L.P.; Pedrosa, M.L.; Peluzio, M.D.C.G. Aerobic exercise and not a diet supplemented with jussara açaí (Euterpe edulis Martius) alters hepatic oxidative and inflammatory biomarkers in ApoE-deficient mice. Br. J. Nutr. 2014, 112, 285-294. [CrossRef]

174. Cordeiro, V.D.S.C.; De Bem, G.F.; Da Costa, C.A.; Santos, I.B.; De Carvalho, L.C.R.M.; Ognibene, D.T.; Da Rocha, A.P.M.; De Carvalho, J.J.; De Moura, R.S.; Resende, A.C. Euterpe oleracea Mart. seed extract protects against renal injury in diabetic and spontaneously hypertensive rats: Role of inflammation and oxidative stress. Eur. J. Nutr. 2017, 57,817-832. [CrossRef]

175. De Souza, M.O.; e Silva, L.S.; Magalhães, C.L.D.B.; De Figueiredo, B.B.; Costa, D.C.; Silva, M.E.; Pedrosa, M.L. The hypocholesterolemic activity of açaí (Euterpe oleracea Mart.) is mediated by the enhanced expression of the ATP-binding cassette, subfamily G transporters 5 and 8 and low-density lipoprotein receptor genes in the rat. Nutr. Res. 2012, 32, 976-984. [CrossRef] [PubMed]

176. De Bem, G.F.; Costa, C.A.; Santos, I.B.; Cordeiro, V.D.S.C.; De Carvalho, L.C.R.M.; De Souza, M.A.V.; Soares, R.D.A.; Sousa, P.J.D.C.; Ognibene, D.T.; Resende, A.C.; et al. Antidiabetic effect of Euterpe oleracea Mart. (açaí) extract and exercise training on high-fat diet and streptozotocin-induced diabetic rats: A positive interaction. PLoS ONE 2018, 13, e0199207. [CrossRef] [PubMed]

177. Guergoletto, K.B.; Costabile, A.; Flores, G.; Garcia, S.; Gibson, G.R. In vitro fermentation of juçara pulp (Euterpe edulis) by human colonic microbiota. Food Chem. 2016, 196, 251-258. [CrossRef] [PubMed]

178. AlQurashi, R.M.; Alarifi, S.N.; Walton, G.E.; Costabile, A.; Rowland, I.R.; Commane, D.M. In vitro approaches to assess the effects of açai (Euterpe oleracea) digestion on polyphenol availability and the subsequent impact on the faecal microbiota. Food Chem. 2017, 234, 190-198. [CrossRef]

179. Guerra, J.F.D.C.; Maciel, P.S.; De Abreu, I.C.M.E.; Pereira, R.R.; Silva, M.; Cardoso, L.D.M.; Pinheiro-Sant'Ana, H.M.; Lima, W.G.; Silva, M.E.; Pedrosa, M.L. Dietary açai attenuates hepatic steatosis via adiponectin-mediated effects on lipid metabolism in high-fat diet mice. J. Funct. Foods 2015, 14, 192-202. [CrossRef]

180. Mertens-Talcott, S.U.; Rios, J.; Jilma-Stohlawetz, P.; Pacheco-Palencia, L.A.; Meibohm, B.; Talcott, S.T.; Derendorf, H. Pharmacokinetics of anthocyanins and antioxidant effects after the consumption of anthocyanin-rich açai juice and Pulp (Euterpe oleracea Mart.) in human healthy volunteers. J. Agric. Food Chem. 2008, 56, 7796-7802. [CrossRef]

181. Udani, J.; Singh, B.B.; Singh, V.J.; Barrett, M.L. Effects of Açai (Euterpe oleracea Mart.) berry preparation on metabolic parameters in a healthy overweight population: A pilot study. Nutr. J. 2011, 10, 45. [CrossRef]

182. Yamane, T.; Kozuka, M.; Konda, D.; Nakano, Y.; Nakagaki, T.; Ohkubo, I.; Ariga, H. Improvement of blood glucose levels and obesity in mice given aronia juice by inhibition of dipeptidyl peptidase IV and

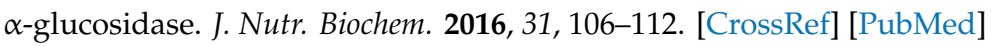

183. Jeon, Y.D.; Kang, S.H.; Moon, K.H.; Lee, J.H.; Kim, D.G.; Kim, W.; Kim, J.S.; Ahn, B.Y.; Jin, J.S. the effect of aronia berry on Type 1 Diabetes in vivo and in vitro. J. Med. Food 2018, 21, 244-253. [CrossRef]

184. Park, C.H.; Kim, J.H.; Lee, E.B.; Hur, W.; Kwon, O.-J.; Park, H.-J.; Yoon, S.K. Aronia melanocarpa extract ameliorates hepatic lipid metabolism through PPAR 2 downregulation. PLoS ONE 2017, 12, e0169685. [CrossRef] [PubMed]

185. Kim, S.; Baek, S.-K.; Bin Song, K. Physical and antioxidant properties of alginate films prepared from Sargassum fulvellum with black chokeberry extract. Food Packag. Shelf Life 2018, 18, 157-163. [CrossRef]

186. Valcheva-Kuzmanova, S.; Kuzmanov, K.; Tancheva, S.; Belcheva, A. Hypoglycemic effects of Aronia melanocarpa fruit juice in streptozotocin-induced diabetic rats. Methods Find. Exp. Clin. Pharmacol. 2007, 29, 101-105. [CrossRef]

187. Lipinska, P.; Atanasov, A.G.; Palka, M.; Jóźwik, A. Chokeberry pomace as a determinant of antioxidant parameters assayed in blood and liver tissue of polish merino and Wrzosówka lambs. Molecules 2017, 22, 1461. [CrossRef]

188. Sikora, J.; Broncel, M.; Markowicz, M.; Chałubiński, M.; Wojdan, K.; Mikiciuk-Olasik, E. Short-term supplementation with Aronia melanocarpa extract improves platelet aggregation, clotting, and fibrinolysis in patients with metabolic syndrome. Eur. J. Nutr. 2011, 51, 549-556. [CrossRef] 
189. Jurgoński, A.; Juskiewicz, J.; Zduńczyk, Z. Ingestion of black chokeberry fruit extract leads to intestinal and systemic changes in a rat model of prediabetes and hyperlipidemia. Plant. Foods Hum. Nutr. 2008, 63, 176-182. [CrossRef]

190. Kujawska, M.; Ignatowicz, E.; Ewertowska, M.; Oszmiański, J.; Jodynis-Liebert, J. Protective effect of chokeberry on chemical-induced oxidative stress in rat. Hum. Exp. Toxicol. 2010, 30, 199-208. [CrossRef]

191. Bhaswant, M.; Shafie, S.R.; Mathai, M.L.; Mouatt, P.; Brown, L. Anthocyanins in chokeberry and purple maize attenuate diet-induced metabolic syndrome in rats. Nutrients 2017, 41, 24-31. [CrossRef]

192. Qin, B.; Anderson, R.A. An extract of chokeberry attenuates weight gain and modulates insulin, adipogenic and inflammatory signalling pathways in epididymal adipose tissue of rats fed a fructose-rich diet. Br. J. Nutr. 2011, 108, 581-587. [CrossRef] [PubMed]

193. Jakovljevic, V.; Milic, P.; Bradic, J.; Jeremic, J.; Zivkovic, V.; Srejovic, I.M.; Turnic, T.R.N.; Milosavljevic, I.; Jeremic, N.; Bolevich, S.; et al. Standardized aronia melanocarpa extract as novel supplement against metabolic syndrome: A rat model. Int. J. Mol. Sci. 2018, 20, 6. [CrossRef] [PubMed]

194. Milutinović, M.; Branković, S.; Ćujić, N.; Šavikin, K.; Kostić, M.; Kitić, N.; Miladinović, B.; Kitić, D. Antispasmodic effects of black chokeberry (Aronia melanocarpa (Michx.) Elliott) extracts and juice and their potential use in gastrointestinal disorders. J. Berry Res. 2020, 10, 175-192. [CrossRef]

195. Kardum, N.; Konić-Ristić, A.; Šavikin, K.; Spasic, S.; Stefanovic, A.; Ivanisevic, J.; Miljkovic, M. Effects of polyphenol-rich chokeberry juice on antioxidant/pro-oxidant status in healthy subjects. J. Med. Food 2014, 17, 869-874. [CrossRef] [PubMed]

196. Kardum, N.; Takić, M.; Šavikin, K.; Zec, M.; Zdunić, G.; Spasic, S.; Konić-Ristić, A. Effects of polyphenol-rich chokeberry juice on cellular antioxidant enzymes and membrane lipid status in healthy women. J. Funct. Foods 2014, 9, 89-97. [CrossRef]

197. Broncel, M.; Kozirog, M.; Duchnowicz, P.; Koter-Michalak, M.; Sikora, J.; Chojnowska-Jezierska, J. Aronia melanocarpa extract reduces blood pressure, serum endothelin, lipid, and oxidative stress marker levels in patients with metabolic syndrome. Med. Sci. Monit. 2010, 16.

198. Kardum, N.; Milovanović, B.; Šavikin, K.; Zdunić, G.; Mutavdžin, S.; Gligorijević, T.; Spasic, S. Beneficial effects of polyphenol-rich chokeberry juice consumption on blood pressure level and lipid status in hypertensive subjects. J. Med. Food 2015, 18, 1231-1238. [CrossRef]

199. Badescu, M.; Badulescu, O.; Badescu, L.; Ciocoiu, M. Effects ofSambucus nigraandAronia melanocarpaextracts on immune system disorders within diabetes mellitus. Pharm. Biol. 2014, 53, 533-539. [CrossRef]

200. Nowak, D.; Gośliński, M.; Przygoński, K.; Wojtowicz, E. The antioxidant properties of exotic fruit juices from acai, maqui berry and noni berries. Eur. Food Res. Technol. 2018, 244, 1897-1905. [CrossRef]

201. Skoczynska, A.; Poręba, R.; Steinmentz-Beck, A.; Martynowicz, H.; Affelska-Jercha, A.; Turczyn, B.; Wojakowska, A.; Jedrychowska, I. The dependence between urinary mercury concentration and carotid arterial intima-media thickness in workers occupationally exposed to mercury vapour. Int. J. Occup. Med. Environ. Health 2009, 22. [CrossRef]

202. Istas, G.; Wood, E.; Le Sayec, M.; Rawlings, C.; Yoon, J.; Dandavate, V.; Cera, E.D.; Rampelli, S.; Costabile, A.; Fromentin, E.; et al. Effects of aronia berry (poly)phenols on vascular function and gut microbiota: A double-blind randomized controlled trial in adult men. Am. J. Clin. Nutr. 2019, 110, 316-329. [CrossRef]

203. Tani, T.; Nishikawa, S.; Kato, M.; Tsuda, T. Delphinidin 3-rutinoside-rich blackcurrant extract ameliorates glucose tolerance by increasing the release of glucagon-like peptide-1 secretion. Food Sci. Nutr. 2017, 5, 929-933. [CrossRef] [PubMed]

204. Iizuka, Y.; Ozeki, A.; Tani, T.; Tsuda, T. Blackcurrant extract ameliorates hyperglycemia in Type 2 Diabetic mice in association with increased basal secretion of glucagon-like peptide-1 and activation of AMP-activated protein kinase. J. Nutr. Sci. Vitaminol. 2018, 64, 258-264. [CrossRef] [PubMed]

205. Esposito, D.; Damsud, T.; Wilson, M.; Grace, M.H.; Strauch, R.; Li, X.; Lila, M.A.; Komarnytsky, S. Black currant anthocyanins attenuate weight gain and improve glucose metabolism in diet-induced obese mice with intact, but not disrupted, gut microbiome. J. Agric. Food Chem. 2015, 63, 6172-6180. [CrossRef]

206. Benn, T.; Kim, B.; Park, Y.K.; Wegner, C.J.; Harness, E.; Nam, T.G.; Kim, D.O.; Lee, J.S.; Lee, J.Y. Polyphenol-rich blackcurrant extract prevents inflammation in diet-induced obese mice. J. Nutr. Biochem. 2014, 25, 1019-1025. [CrossRef] [PubMed] 
207. Jurgoński, A.; Juskiewicz, J.; Zduńczyk, Z.; Matusevicius, P.; Kołodziejczyk, K. Polyphenol-rich extract from blackcurrant pomace attenuates the intestinal tract and serum lipid changes induced by a high-fat diet in rabbits. Eur. J. Nutr. 2014, 53, 1603-1613. [CrossRef]

208. Molan, A.; Liu, Z.; Kruger, M. The ability of blackcurrant extracts to positively modulate key markers of gastrointestinal function in rats. World, J. Microbiol. Biotechnol. 2010, 26, 1735-1743. [CrossRef]

209. Park, J.H.; Kho, M.C.; Kim, H.Y.; Ahn, Y.M.; Lee, Y.J.; Kang, D.G.; Lee, H.S. Blackcurrant suppresses metabolic syndrome induced by high-fructose diet in rats. Evid. Based Complement. Altern. Med. 2015, 2015, 1-11. [CrossRef]

210. Paturi, G.; Butts, C.A.; Monro, J.A.; Hedderley, D.I. Effects of blackcurrant and dietary fibers on large intestinal health biomarkers in rats. Plant. Foods Hum. Nutr. 2018, 73, 54-60. [CrossRef]

211. Molan, A.; Liu, Z.; Plimmer, G. Evaluation of the effect of blackcurrant products on gut microbiota and on markers of risk for colon cancer in humans. Phytother. Res. 2013, 28, 416-422. [CrossRef]

212. Castro-Acosta, M.L.; Stone, S.G.; Mok, J.E.; Mhajan, R.K.; Fu, C.I.; Lenihan-Geels, G.N.; Corpe, C.P.; Hall, W. Apple and blackcurrant polyphenol-rich drinks decrease postprandial glucose, insulin and incretin response to a high-carbohydrate meal in healthy men and women. J. Nutr. Biochem. 2017, 49, 53-62. [CrossRef] [PubMed]

213. Castro-Acosta, M.L.; Smith, L.; Miller, R.J.; McCarthy, D.I.; Farrimond, J.A.; Hall, W. Drinks containing anthocyanin-rich blackcurrant extract decrease postprandial blood glucose, insulin and incretin concentrations. J. Nutr. Biochem. 2016, 38, 154-161. [CrossRef] [PubMed]

214. Willems, M.; Parktin, N.; Widjaja, W.; Ajjimaporn, A. Effect of New Zealand blackcurrant extract on physiological responses at rest and during brisk walking in southeast asian men: A randomized, double-blind, placebo-controlled, crossover study. Nutrients 2018, 10, 1732. [CrossRef] [PubMed]

215. Strauss, J.A.; Willems, M.E.; Shepherd, S.O. New Zealand blackcurrant extract enhances fat oxidation during prolonged cycling in endurance-trained females. Eur. J. Appl. Physiol. 2018, 118, 1265-1272. [CrossRef] [PubMed]

216. Lomiwes, D.; Ha, B.; Ngametua, N.; Burr, N.; Cooney, J.M.; Trower, T.; Sawyer, G.M.; Hedderley, D.I.; Hurst, R.D.; Hurst, S.M. Timed consumption of a New Zealand blackcurrant juice support positive affective responses during a self-motivated moderate walking exercise in healthy sedentary adults. J. Int. Soc. Sports Nutr. 2019, 16, 1-14. [CrossRef] [PubMed]

217. Hurst, R.D.; Lyall, K.A.; Roberts, J.M.; Perthaner, A.; Wells, R.W.; Cooney, J.M.; Jensen, D.J.; Burr, N.; Hurst, S.M. Consumption of an anthocyanin-rich extract made from new zealand blackcurrants prior to exercise may assist recovery from oxidative stress and maintains circulating neutrophil function: A pilot study. Front. Nutr. 2019, 6, 73. [CrossRef]

218. Alvarado, J.L.; Leschot, A.; Olivera-Nappa, Á.; Salgado, A.-M.; Rioseco, H.; Lyon, C.; Vigil, P. Delphinidin-rich maqui berry extract (Delphinol ${ }^{\circledR}$ ) lowers fasting and postprandial glycemia and insulinemia in prediabetic individuals during oral glucose tolerance tests. BioMed Res. Int. 2016, 2016, 9070537. [CrossRef] [PubMed]

219. Di Lorenzo, A.; Sobolev, A.P.; Nabavi, S.; Sureda, A.; Moghaddam, A.; Khanjani, S.; Di Giovanni, C.; Xiao, J.; Shirooie, S.; Sokeng, A.J.T; et al. Corrigendum to Antidepressive effects of a chemically characterized maqui berry extract (Aristotelia chilensis (molina) stuntz) in a mouse model of Post-stroke depression. Food Chem. Toxicol. 2019, 131, 110641. [CrossRef]

220. Rojo, L.E.; Ribnicky, D.; Logendra, S.; Poulev, A.; Rojas-Silva, P.; Kuhn, P.; Dorn, R.; Grace, M.H.; Lila, M.A.; Raskin, I. In vitro and in vivo anti-diabetic effects of anthocyanins from maqui berry (Aristotelia chilensis). Food Chem. 2011, 131, 387-396. [CrossRef]

221. Davinelli, S.; Bertoglio, J.C.; Zarrelli, A.; Pina, R.; Scapagnini, G. A Randomized clinical trial evaluating the efficacy of an anthocyanin-maqui berry extract (Delphinol ${ }^{\circledR}$ ) on oxidative stress biomarkers. J. Am. Coll. Nutr. 2015, 34, 28-33. [CrossRef]

222. Parra-Vargas, M.; Sandoval-Rodríguez, A.S.; Rodriguez-Echevarria, R.; Dominguez-Rosales, J.A.; Santos-Garcia, A.; Armendariz-Borunda, J. Delphinidin ameliorates hepatic triglyceride accumulation in human hepg2 cells, but not in diet-induced obese mice. Nutrients 2018, 10, 1060. [CrossRef] [PubMed]

223. Corsi, R.; Mosti, G.; Cavezzi, A.; Urso, S.U.; Dimitrova, G.; Fioroni, E.; Colucci, R.; Quinzi, V. A polyphenol-based multicomponent nutraceutical in dysmetabolism and oxidative stress: Results from a pilot study. J. Diet. Suppl. 2017, 15, 34-41. [CrossRef] [PubMed] 
224. Ávila, F.; Jiménez-Aspee, F.; Cruz, N.; Gómez, C.; González, M.A.; Ravello, N. Additive effect of maqui (Aristotelia chilensis) and lemon (Citrus $\mathrm{x}$ limon) juice in the postprandial glycemic responses after the intake of high glycemic index meals in healthy men. NFS J. 2019, 17, 8-16. [CrossRef]

225. Sandoval, V.; Femenias, A.; Martínez-Garza, Ú.; Sanz-Lamora, H.; Castagnini, J.M.; Quifer-Rada, P.; Lamuela-Raventós, R.-M.; Marrero, P.F.; Haro, D.; Relat, J. Lyophilized maqui (Aristotelia chilensis) berry induces browning in the subcutaneous white adipose tissue and ameliorates the insulin resistance in high fat diet-induced obese mice. Antioxidants 2019, 8, 360. [CrossRef]

226. Wang, W.; Yagiz, Y.; Buran, T.J.; Nunes, C.D.N.; Gu, L. Phytochemicals from berries and grapes inhibited the formation of advanced glycation end-products by scavenging reactive carbonyls. Food Res. Int. 2011, 44, 2666-2673. [CrossRef]

227. Barrett, A.; Ndou, T.; Hughey, C.A.; Straut, C.; Howell, A.; Dai, Z.; Kaletunc, G. Inhibition of $\alpha$-amylase and glucoamylase by tannins extracted from cocoa, pomegranates, cranberries, and grapes. J. Agric. Food Chem. 2013, 61, 1477-1486. [CrossRef]

228. Pinto, M.D.S.; Ghaedian, R.; Shinde, R.; Shetty, K. Potential of cranberry powder for management of hyperglycemia using in vitro models. J. Med. Food 2010, 13, 1036-1044. [CrossRef]

229. Pappas, E.; Schaich, K.M. Phytochemicals of cranberries and cranberry products: Characterization, potential health effects, and processing stability. Crit. Rev. Food Sci. Nutr. 2009, 49, 741-781. [CrossRef]

230. Gulcin, I.; Topal, F.; Çakmakçı, R.; Bílsel, M.; Goren, A.C.; Erdogan, U.; Cakmakci, R. Pomological features, nutritional quality, polyphenol content analysis, and antioxidant properties of domesticated and 3 wild ecotype forms of raspberries (Rubus idaeus L.). J. Food Sci. 2011, 76, C585-C593. [CrossRef]

231. Sparzak, B. HPLC analysis of polyphenols in the fruits of Rubus idaeus L. (Rosaceae). Nat. Prod. Res. 2010, 24, 1811-1822. [CrossRef]

232. Krauze-Baranowska, M.; Głód, D.; Kula, M.; Majdan, M.; Hałasa, R.; Matkowski, A.; Kozłowska, W.; Kawiak, A. chemical composition and biological activity of Rubus idaeus shoots-A traditional herbal remedy of Eastern Europe. BMC Complement. Altern. Med. 2014, 14, 480. [CrossRef] [PubMed]

233. Mena, P.; Tassotti, M.; Martínez, J.J.; Hernández, F.; Del Rio, D.; Sánchez-Salcedo, E.M. Phytochemical evaluation of eight white (Morus alba L.) and black (Morus nigra L.) mulberry clones grown in Spain based on UHPLC-ESI-MSn metabolomic profiles. Food Res. Int. 2016, 89, 1116-1122. [CrossRef]

234. Natić, M.; Dabić, D.Č.; Papetti, A.; Akšić, M.M.F.; Ognjanov, V.; Ljubojević, M.; Tešić, Ž.L. Analysis and characterisation of phytochemicals in mulberry (Morus alba L.) fruits grown in Vojvodina, North Serbia. Food Chem. 2015, 171, 128-136. [CrossRef]

235. Jin, Q.; Yang, J.; Ma, L.; Cai, J.; Li, J. Comparison of polyphenol profile and inhibitory activities against oxidation and $\alpha$-glucosidase in mulberry (Genus Morus) cultivars from China. J. Food Sci. 2015, 80, C2440-C2451. [CrossRef]

236. Bujor, O.-C.; Giniès, C.; Popa, V.I.; Dufour, C. Phenolic compounds and antioxidant activity of lingonberry (Vaccinium vitis-idaea L.) leaf, stem and fruit at different harvest periods. Food Chem. 2018, 252, 356-365. [CrossRef]

237. Ieri, F.; Martini, S.; Innocenti, M.; Mulinacci, N. Phenolic distribution in liquid preparations of Vaccinium myrtillus L. and Vaccinium vitis idaea L. Phytochem. Anal. 2013, 24, 467-475. [CrossRef]

238. Spínola, V.; Pinto, J.; Castilho, P. Hypoglycemic, anti-glycation and antioxidant in vitro properties of two Vaccinium species from Macaronesia: A relation to their phenolic composition. J. Funct. Foods 2018, 40, 595-605. [CrossRef]

239. Spínola, V.; Pinto, J.; Llorent-Martínez, E.J.; Tomás, H.; Castilho, P. Evaluation of Rubus grandifolius L. (wild blackberries) activities targeting management of type-2 diabetes and obesity using in vitro models. Food Chem. Toxicol. 2019, 123, 443-452. [CrossRef]

240. Aaby, K.; Ekeberg, D.; Skrede, G. Characterization of phenolic compounds in strawberry (Fragaria $\times$ ananassa) fruits by different HPLC detectors and contribution of individual compounds to total antioxidant Capacity. J. Agric. Food Chem. 2007, 55, 4395-4406. [CrossRef]

241. Šaponjac, V.T.; Gironés-Vilaplana, A.; Djilas, S.; Mena, P.; Ćetković, G.; Moreno, D.A.; Čanadanović-Brunet, J.; Vulić, J.; Stajčić, S.; Vinčić, M. Chemical composition and potential bioactivity of strawberry pomace. RSC Adv. 2015, 5, 5397-5405. [CrossRef]

242. Wojdyło, A.; Nowicka, P.; Babelewski, P. Phenolic and carotenoid profile of new goji cultivars and their anti-hyperglycemic, anti-aging and antioxidant properties. J. Funct. Foods 2018, 48, 632-642. [CrossRef] 
243. Montesano, D.; Rocchetti, G.; Cossignani, L.; Lucini, L.; Simonetti, M.S.; Blasia, F. Italian Lycium barbarum L. berry: Chemical characterization and nutraceutical value. Nat. Prod. Commun. 2018, 13. [CrossRef]

244. Schauss, A.G.; Wu, X.; Prior, R.L.; Ou, B.; Huang, D.-J.; Owens, J.; Agarwal, A.; Jensen, G.S.; Hart, A.N.; Shanbrom, E. Antioxidant capacity and other bioactivities of the freeze-dried amazonian palm berry, euterpe oleraceaemart. (Acai). J. Agric. Food Chem. 2006, 54, 8604-8610. [CrossRef] [PubMed]

245. Schauss, A.G.; Wu, X.; Prior, R.L.; Ou, B.; Patel, D.; Huang, D.-J.; Kababick, J.P. Phytochemical and nutrient composition of the freeze-dried amazonian palm berry, euterpe oleraceaemart. (Acai). J. Agric. Food Chem. 2006, 54, 8598-8603. [CrossRef] [PubMed]

246. Pacheco-Palencia, L.A. Absorption and biological activity of phytochemical-rich extracts from açai (Euterpe oleracea Mart.) pulp and oil in vitro. J. Agric. Food Chem. 2008, 56, 3593-3600. [CrossRef]

247. Sosnowska, D. Inhibitory effect of black chokeberry fruit polyphenols on pancreatic lipase-Searching for most active inhibitors. J. Funct. Foods 2018, 49, 196-204. [CrossRef]

248. Anttonen, M.J.; Karjalainen, R.O. High-performance liquid chromatography analysis of black currant (Ribes nigrum L.) fruit phenolics grown either conventionally or organically. J. Agric. Food Chem. 2006, 54, 7530-7538. [CrossRef]

249. Borges, G.; Degeneve, A.; Mullen, W.; Crozier, A. Identification of flavonoid and phenolic antioxidants in black currants, blueberries, raspberries, red currants, and cranberries. J. Agric. Food Chem. 2010, 58, 3901-3909. [CrossRef]

250. Cespedes, C.L.; Pavon, N.; Dominguez, M.; Alarcon, J.; Balbontin, C.; Kubo, I.; El-Hafidi, M.; Avila, J.G.; Dominguez-Lopez, M. The chilean superfruit black-berry Aristotelia chilensis (Elaeocarpaceae), Maqui as mediator in inflammation-associated disorders. Food Chem. Toxicol. 2017, 108, 438-450. [CrossRef]

251. Escribano-Bailón, M.T.; Alcalde-Eon, C.; Muñoz, O.; Rivas-Gonzalo, J.C.; Santos-Buelga, C. Anthocyanins in berries of Maqui [Aristotelia chilensis (Mol.) Stuntz]. Phytochem. Anal. 2005, 17, 8-14. [CrossRef]

252. Häkkinen, S.H.; Kärenlampi, S.O.; Mykkänen, H.M.; Törrönen, A.R. Influence of domestic processing and storage on flavonol contents in berries. J. Agric. Food Chem. 2000, 48, 2960-2965. [CrossRef] [PubMed]

253. Ferrier, J.; Djeffal, S.; Morgan, H.P.; Kloet, S.P.V.; Redžić, S.; Cuerrier, A.; Balick, M.J.; Arnason, J.T. Antiglycation activity of Vaccinium spp. (Ericaceae) from the Sam Vander Kloet collection for the treatment of type II diabetes1This article is part of a Special Issue entitled 'A tribute to Sam Vander Kloet FLS: Pure and applied research from blueberries to heathland ecology'. Botany 2012, 90, 401-406. [CrossRef]

254. Buchert, J.; Koponen, J.M.; Suutarinen, M.; Mustranta, A.; Lille, M.E.; Törrönen, R.; Poutanen, K. Effect of enzyme-aided pressing on anthocyanin yield and profiles in bilberry and blackcurrant juices. J. Sci. Food Agric. 2005, 85, 2548-2556. [CrossRef]

255. Cermak, R.; Landgraf, S.; Wolffram, S. Quercetin glucosides inhibit glucose uptake into brush-border-membrane vesicles of porcine jejunum. Br. J. Nutr. 2004, 91, 849-855. [CrossRef]

256. Morazzoni, P.; Livio, S.; Scilingo, A.; Malandrino, S. Vaccinium myrtillus anthocyanosides pharmacokinetics in rats. Arzneimittelforschung 1991, 41, 128-131.

257. Liu, H.; Liu, H.; Wang, W.; Khoo, C.; Taylor, J.; Gu, L. Cranberry phytochemicals inhibit glycation of human hemoglobin and serum albumin by scavenging reactive carbonyls. Food Funct. 2011, 2, 475. [CrossRef] [PubMed]

258. Podsędek, A.; Majewska, I.; Redzynia, M.; Sosnowska, D.; Koziolkiewicz, M. In vitro inhibitory effect on digestive enzymes and antioxidant potential of commonly consumed fruits. J. Agric. Food Chem. 2014, 62, 4610-4617. [CrossRef] [PubMed]

259. Sun, J.; Liu, W.; Ma, H.; Marais, J.P.J.; Khoo, C.; Dain, J.A.; Rowley, D.C.; Seeram, N.P. Effect of cranberry (Vaccinium macrocarpon) oligosaccharides on the formation of advanced glycation end-products. J. Berry Res. 2016, 6, 149-158. [CrossRef] [PubMed]

260. Wilson, T.; Bauer, B.A. Advising consumers about dietary supplements: Lessons from cranberry products. J. Diet. Suppl. 2009, 6, 377-384. [CrossRef] [PubMed]

261. Kaur, G.; Rao, L.V.M.; Agrawal, A.; Pendurthi, U.R. Effect of wine phenolics on cytokine-induced C-reactive protein expression. J. Thromb. Haemost. 2007, 5, 1309-1317. [CrossRef]

262. Marathe, P.H.; Gao, H.X.; Close, K.L. American diabetes association standards of medical care in diabetes 2017. J. Diabetes 2017, 9, 320-324. [CrossRef] [PubMed]

263. Grussu, D.; Stewart, D.; McDougall, G.J. Berry polyphenols inhibit $\alpha$-amylasein Vitro: Identifying active components in rowanberry and raspberry. J. Agric. Food Chem. 2011, 59, 2324-2331. [CrossRef] [PubMed] 
264. Tsuda, T.; Ueno, Y.; Yoshikawa, T.; Kojo, H.; Osawa, T. Microarray profiling of gene expression in human adipocytes in response to anthocyanins. Biochem. Pharmacol. 2006, 71, 1184-1197. [CrossRef] [PubMed]

265. Al-Salmi, F.A. Ameliorative and synergistic effect of red raspberry and lycopene against hepatotoxicity induced by acrylamide in male mice. Int. J. Pharmacol. 2019, 15, 166-176. [CrossRef]

266. Zou, T.; Kang, Y.; Wang, B.; De Avila, J.M.; You, J.; Zhu, M.-J.; Du, M. Raspberry supplementation reduces lipid accumulation and improves insulin sensitivity in skeletal muscle of mice fed a high-fat diet. J. Funct. Foods 2019, 63, 103572. [CrossRef]

267. Park, K.S. Raspberry ketone, a naturally occurring phenolic compound, inhibits adipogenic and lipogenic gene expression in 3T3-L1 adipocytes. Pharm. Biol. 2014, 53, 870-875. [CrossRef]

268. Lee, H.; Kim, J.; An, J.; Lee, S.; Kong, H.; Song, Y.; Choi, H.R.; Lee, S.J.; Chae, K.S.; Kwon, J.W.; et al. Amelioration of hyperglycemia by Rubus occidentalis (black raspberry) and increase in short-chain fatty acids producing bacteria. J. Funct. Foods 2019, 54, 433-439. [CrossRef]

269. Yan, F.; Chen, Y.; Azat, R.; Zheng, X. Mulberry anthocyanin extract ameliorates oxidative damage in HepG2 cells and prolongs the lifespan of caenorhabditis elegans through MAPK and Nrf2 pathways. Oxidative Med. Cell. Longev. 2017, 2017, 1-12. [CrossRef]

270. He, X.; Fang, J.; Ruan, Y.; Wang, X.; Sun, Y.; Wu, N.; Zhao, Z.; Chang, Y.; Ning, N.; Guo, H.; et al. Structures, bioactivities and future prospective of polysaccharides from Morus alba (white mulberry): A review. Food Chem. 2018, 245, 899-910. [CrossRef]

271. He, H.; Yu, W.G.; Yang, J.P.; Ge, S.; Lu, Y.H. Multiple Comparisons of glucokinase activation mechanisms of five mulberry bioactive ingredients in hepatocyte. J. Agric. Food Chem. 2015, 64, 2475-2484. [CrossRef]

272. Yan, F.; Dai, G.; Zheng, X. Mulberry anthocyanin extract ameliorates insulin resistance by regulating PI3K/AKT pathway in HepG2 cells and db/db mice. J. Nutr. Biochem. 2016, 36, 68-80. [CrossRef] [PubMed]

273. Wang, Y.; Xiang, L.; Wang, C.; Tang, C.; He, X. Antidiabetic and antioxidant effects and phytochemicals of mulberry fruit (Morus alba L.) polyphenol enhanced extract. PLoS ONE 2013, 8, e71144. [CrossRef] [PubMed]

274. Zhang, H.; Ma, Z.F.; Luo, X.; Li, X. Effects of mulberry fruit (Morus alba L.) Consumption on health outcomes: A mini-review. Antioxidants 2018, 7, 69. [CrossRef] [PubMed]

275. Hu, D.; Bao, T.; Lu, Y.; Su, H.; Ke, H.; Chen, W. Polysaccharide from Mulberry Fruit (Morus alba L.) Protects against palmitic-acid-induced hepatocyte lipotoxicity by activating the Nrf2/ARE signaling pathway. J. Agric. Food Chem. 2019. [CrossRef]

276. Harbilas, D.; Martineau, L.C.; Harris, C.S.; Adeyiwola-Spoor, D.C.; Saleem, A.; Lambert, J.; Caves, D.; Johns, T.; Prentki, M.; Cuerrier, A.; et al. Evaluation of the antidiabetic potential of selected medicinal plant extracts from the Canadian boreal forest used to treat symptoms of diabetes: Part II. Can. J. Physiol. Pharmacol. 2009, 87, 479-492. [CrossRef]

277. Ross, K.A.; Godfrey, D.; Fukumoto, L. The chemical composition, antioxidant activity and -glucosidase inhibitory activity of water-extractable polysaccharide conjugates from northern Manitoba lingonberry. Cogent Food Agric. 2015, 1. [CrossRef]

278. Quek, R.; Henry, C.J. Influence of polyphenols from lingonberry, cranberry, and red grape onin vitrodigestibility of rice. Int. J. Food Sci. Nutr. 2015, 66, 378-382. [CrossRef]

279. Beaulieu, L.-P.; Harris, C.S.; Saleem, A.; Cuerrier, A.; Haddad, P.S.; Martineau, L.C.; Bennett, S.A.; Arnason, J.T. Inhibitory effect of the cree traditional medicine wiishichimanaanh (Vaccinium vitis-idaea) on advanced glycation endproduct formation: Identification of active principles. Phytotherapy Res. 2009, 24, 741-747. [CrossRef]

280. Kylli, P.; Nohynek, L.; Puupponen-Pimia, R.; Westerlund-Wikström, B.; Leppanen, T.; Welling, J.; Moilanen, E.; Heinonen, M. Lingonberry (Vaccinium vitis-idaea) and European cranberry (Vaccinium microcarpon) proanthocyanidins: Isolation, identification, and bioactivities. J. Agric. Food Chem. 2011, 59, 3373-3384. [CrossRef]

281. Kowalska, K.; Olejnik, A.; Zielińska-Wasielica, J.; Olkowicz, M. Inhibitory effects of lingonberry (Vaccinium vitis-idaea L.) fruit extract on obesity-induced inflammation in 3T3-L1 adipocytes and RAW 264.7 macrophages. J. Funct. Foods 2019, 54, 371-380. [CrossRef]

282. Riihinen, K.; Kamal-Eldin, A.; Mattila, P.; González-Paramás, A.M.; Törrönen, A.R. Distribution and contents of phenolic compounds in eighteen Scandinavian berry species. J. Agric. Food Chem. 2004, 52, 4477-4486. [CrossRef] [PubMed] 
283. Hildebrandt, M.A.; Hoffmann, C.; Sherrill-Mix, S.A.; Keilbaugh, S.A.; Hamady, M.; Chen, Y.; Knight, R.; Ahima, R.S.; Bushman, F.D.; Wu, G.D.; et al. High-fat diet determines the composition of the murine gut microbiome independently of obesity. Gastroenterology 2009, 137, 1716-1724. [CrossRef] [PubMed]

284. Krych, L.; Nielsen, D.; Hansen, A.; Hansen, C. Gut microbial markers are associated with diabetes onset, regulatory imbalance, and IFN- $\gamma$ level in NOD Mice. Gut Microbes 2015, 6, 101-109. [CrossRef] [PubMed]

285. Kameyama, K.; Itoh, K. Intestinal Colonization by a Lachnospiraceae Bacterium Contributes to the Development of Diabetes in Obese Mice. Microbes Environ. 2014, 29, 427-430. [CrossRef] [PubMed]

286. Everard, A.; Belzer, C.; Geurts, L.; Ouwerkerk, J.P.; Druart, C.; Bindels, L.B.; Guiot, Y.; Derrien, M.; Muccioli, G.G.; Delzenne, N.M.; et al. Cross-talk between Akkermansia muciniphila and intestinal epithelium controls diet-induced obesity. Proc. Natl. Acad. Sci. USA 2013, 110, 9066-9071. [CrossRef] [PubMed]

287. Sarkar, D.; Orwat, J.; Hurburt, T.; Woods, F.; Pitts, J.A.; Shetty, K. Evaluation of phenolic bioactive-linked functionality of blackberry cultivars targeting dietary management of early stages type-2 diabetes using in vitro models. Sci. Hortic. 2016, 212, 193-202. [CrossRef]

288. Sancho, R.A.S.; Pastore, G.M. Evaluation of the effects of anthocyanins in type 2 diabetes. Food Res. Int. 2012, 46, 378-386. [CrossRef]

289. Boath, A.S.; Grussu, D.; Stewart, D.; McDougall, G.J. Berry polyphenols inhibit digestive enzymes: A source of potential health benefits? Food Dig. 2012, 3, 1-7. [CrossRef]

290. Gowd, V.; Bao, T.; Chen, W. Antioxidant potential and phenolic profile of blackberry anthocyanin extract followed by human gut microbiota fermentation. Food Res. Int. 2018, 120, 523-533. [CrossRef]

291. Solverson, P.; Rumpler, W.V.; Leger, J.L.; Redan, B.W.; Ferruzzi, M.; Baer, D.J.; Castonguay, T.W.A.; Novotny, J. Blackberry feeding increases fat oxidation and improves insulin sensitivity in overweight and obese males. Nutrients 2018, 10, 1048. [CrossRef]

292. Williamson, G. Possible effects of dietary polyphenols on sugar absorption and digestion. Mol. Nutr. Food Res. 2012, 57, 48-57. [CrossRef] [PubMed]

293. Giampieri, F.; Forbes-Hernández, T.Y.; Gasparrini, M.; Alvarez-Suarez, J.M.; Afrin, S.; Bompadre, S.; Quiles, J.L.; Mezzetti, B.; Battino, M. Strawberry as a health promoter: An evidence based review. Food Funct. 2015, 6, 1386-1398. [CrossRef] [PubMed]

294. Zhu, Q. In vitro bioactivities and phytochemical profile of various parts of the strawberry (Fragaria $\times$ ananassa var. Amaou). J. Funct. Foods 2015, 13, 38-49. [CrossRef]

295. Manzano, S.; Williamson, G. Polyphenols and phenolic acids from strawberry and apple decrease glucose uptake and transport by human intestinal Caco-2 cells. Mol. Nutr. Food Res. 2010, 54, 1773-1780. [CrossRef]

296. da Silva Pinto, M. Evaluation of antiproliferative, anti-Type 2 Diabetes, and antihypertension potentials of ellagitannins from strawberries (Fragaria $\times$ ananassa Duch.) using in vitro models. J. Med. Food 2010, 13, 1027-1035. [CrossRef]

297. Forbes-Hernández, T.Y.; Giampieri, F.; Gasparrini, M.; Afrin, S.; Mazzoni, L.; Cordero, M.D.; Mezzetti, B.; Quiles, J.L.; Battino, M. Lipid accumulation in HepG2 cells is attenuated by strawberry extract through AMPK activation. Nutrients 2017, 9, 621. [CrossRef]

298. Basu, A.; Fu, D.X.; Wilkinson, M.; Simmons, B.; Wu, M.; Betts, N.M.; Du, M.; Lyons, T.J. Strawberries decrease atherosclerotic markers in subjects with metabolic syndrome. Nutr. Res. 2010, 30, 462-469. [CrossRef]

299. Pajk, T.; Rezar, V.; Levart, A.; Salobir, J. Efficiency of apples, strawberries, and tomatoes for reduction of oxidative stress in pigs as a model for humans. Nutrients 2006, 22, 376-384. [CrossRef]

300. Kulczyński, B.; Gramza-Michałowska, A. Goji berry (Lycium barbarum): Composition and health effects-A review. Pol. J. Food Nutr. Sci. 2016, 66, 67-75. [CrossRef]

301. Jin, M.; Huang, Q.; Zhao, K.; Shang, P. Biological activities and potential health benefit effects of polysaccharides isolated from Lycium barbarum L. Int. J. Biol. Macromol. 2013, 54, 16-23. [CrossRef]

302. Zou, P.; Yang, X.; Huang, W.W.; Zhao, H.; Wang, J.; Xu, R.B.; Hu, X.L.; Shen, S.Y.; Qin, D. Characterization and bioactivity of polysaccharides obtained from pine cones of pinus koraiensis by graded ethanol precipitation. Molecules 2013, 18, 9933-9948. [CrossRef] [PubMed]

303. Tang, H.L.; Chen, C.; Wang, S.K.; Sun, G.J. Biochemical analysis and hypoglycemic activity of a polysaccharide isolated from the fruit of Lycium barbarum L. Int. J. Biol. Macromol. 2015, 77, 235-242. [CrossRef] [PubMed]

304. Tang, X.; Shen, T.; Jiang, X.; Xia, M.; Sun, X.; Guo, H.; Ling, W. Purified anthocyanins from bilberry and black currant attenuate hepatic mitochondrial dysfunction and steatohepatitis in mice with methionine and choline deficiency. J. Agric. Food Chem. 2015, 63, 552-561. [CrossRef] [PubMed] 
305. Ding, Y.; Yan, Y.; Peng, Y.; Chen, D.; Mi, J.; Lu, L.; Luo, Q.; Li, X.; Zeng, X.; Cao, Y. In vitro digestion under simulated saliva, gastric and small intestinal conditions and fermentation by human gut microbiota of polysaccharides from the fruits of Lycium barbarum. Int. J. Biol. Macromol. 2019, 125, 751-760. [CrossRef]

306. Girones-Vilaplana, A.; Villaño, D.; Moreno, D.A.; García-Viguera, C. New isotonic drinks with antioxidant and biological capacities from berries (maqui, açaí and blackthorn) and lemon juice. Int. J. Food Sci. Nutr. 2013, 64, 897-906. [CrossRef]

307. Cheguru, P.; Chapalamadugu, K.C.; Doumit, M.E.; Murdoch, G.K.; Hill, R.A. Adipocyte differentiation-specific gene transcriptional response to C18 unsaturated fatty acids plus insulin. Pflugers Arch. 2012, 463, 429-447. [CrossRef]

308. Costa, A.G.V.; Garcia-Diaz, D.F.; Jimenez, P.; Silva, P.I. Bioactive compounds and health benefits of exotic tropical red-black berries. J. Funct. Foods 2013, 5, 539-549. [CrossRef]

309. Karpac, J.; Jasper, H. Insulin and JNK: Optimizing metabolic homeostasis and lifespan. Trends Endocrinol. Metab. 2009, 20, 100-106. [CrossRef]

310. Smith, A.C.; Mullen, K.L.; Junkin, K.A.; Nickerson, J.; Chabowski, A.; Bonen, A.; Dyck, D.J. Metformin and exercise reduce muscle FAT/CD36 and lipid accumulation and blunt the progression of high-fat diet-induced hyperglycemia. Am. J. Physiol. Metab. 2007, 293, E172-E181. [CrossRef]

311. Umpierrez, G.E.; Bailey, T.S.; Carcia, D.; Shaefer, C.; Shubrook, J.H.; Skolnik, N. Improving postprandial hyperglycemia in patients with type 2 diabetes already on basal insulin therapy: Review of current strategies. J. Diabetes 2017, 10, 94-111. [CrossRef]

312. Oyama, L.M.; Silva, F.; Carnier, J.; De Miranda, D.A.; Santamarina, A.B.; Ribeiro, E.B.; Nascimento, C.M.O.D.; De Rosso, V.V. Juçara pulp supplementation improves glucose tolerance in mice. Diabetol. Metab. Syndr. 2016, 8, 8. [CrossRef] [PubMed]

313. Machado, A.K.; Cadoná, F.C.; Assmann, C.E.; Andreazza, A.C.; Duarte, M.M.M.F.; Branco, C.D.S.; Zhou, X.; De Souza, D.V.; Ribeiro, E.E.; Da Cruz, I.B.M. Açaí (Euterpe oleracea Mart.) has anti-inflammatory potential through NLRP3-inflammasome modulation. J. Funct. Foods 2019, 56, 364-371. [CrossRef]

314. Martino, H.S.D.; Dias, M.M.D.S.; Noratto, G.; Talcott, S.; Mertens-Talcott, S.U. Anti-lipidaemic and anti-inflammatory effect of açai (Euterpe oleracea Martius) polyphenols on 3T3-L1 adipocytes. J. Funct. Foods 2016, 23, 432-443. [CrossRef]

315. Du, X.; Myracle, A.D. Fermentation alters the bioaccessible phenolic compounds and increases the alpha-glucosidase inhibitory effects of aronia juice in a dairy matrix followingin vitrodigestion. Food Funct. 2018, 9, 2998-3007. [CrossRef]

316. Rugina, D.; Diaconeasa, Z.M.; Isvoranu, C.; Bunea, A.; Socaciu, C.; Pintea, A. Chokeberry Anthocyanin Extract as Pancreatic $\beta$-Cell Protectors in Two Models of Induced Oxidative Stress. Oxidative Med. Cell. Longev. 2015, 2015, 1-10. [CrossRef]

317. Iwashima, T.; Kudome, Y.; Kishimoto, Y.; Saita, E.; Tanaka, M.; Taguchi, C.; Hirakawa, S.; Mitani, N.; Kondo, K.; Iida, K. Aronia berry extract inhibits TNF- $\alpha$-induced vascular endothelial inflammation through the regulation of STAT3. Food Nutr. Res. 2019, 63, 3361. [CrossRef]

318. Zapolska-Downar, D.; Bryk, D.; Małecki, M.; Hajdukiewicz, K.; Sitkiewicz, D. Aronia melanocarpa fruit extract exhibits anti-inflammatory activity in human aortic endothelial cells. Eur. J. Nutr. 2011, 51, 563-572. [CrossRef]

319. Törrönen, R.; Sarkkinen, E.; Tapola, N.; Hautaniemi, E.; Kilpi, K.; Niskanen, L. Berries modify the postprandial plasma glucose response to sucrose in healthy subjects. Br. J. Nutr. 2009, 103, 1094-1097. [CrossRef]

320. Xu, Y.; Niu, X.; Liu, N.; Gao, Y.; Wang, L.; Xu, G.; Li, X.; Yang, Y. Characterization, antioxidant and hypoglycemic activities of degraded polysaccharides from blackcurrant (Ribes nigrum L.) fruits. Food Chem. 2017, 243, 26-35. [CrossRef]

321. Hossain, A.K.M.M.; Mason, S.L.; Guo, X.-B.; Brennan, C.R. The combined effect of blackcurrant powder and wholemeal flours to improve health promoting properties of cookies. Plant. Foods Hum. Nutr. 2017, 72, 280-287. [CrossRef]

322. Cespedes, C.L.; Valdez-Morales, M.; Avila, J.G.; El-Hafidi, M.; Alarcon, J.; Paredes-López, O. Phytochemical profile and the antioxidant activity of Chilean wild black-berry fruits, Aristotelia chilensis (Mol) Stuntz (Elaeocarpaceae). Food Chem. 2010, 119, 886-895. [CrossRef] 
323. Brauch, J.E.; Reuter, L.; Conrad, J.; Vogel, H.; Schweiggert, R.; Carle, R. Characterization of anthocyanins in novel Chilean maqui berry clones by HPLC-DAD-ESI/MSn and NMR-spectroscopy. J. Food Compos. Anal. 2017, 58, 16-22. [CrossRef]

324. Rubilar, M.; Jara, C.; Poo, Y.; Acevedo, F.; Gutiérrez, C.; Sineiro, J.; Shene, C. Extracts of Maqui (Aristotelia chilensis) and Murta (Ugni molinae Turcz.): Sources of antioxidant compounds and $\alpha$-glucosidase/ $\alpha$-amylase inhibitors. J. Agric. Food Chem. 2011, 59, 1630-1637. [CrossRef] [PubMed]

325. Reyes-Farias, M.; Vasquez, K.; Fuentes, F.; Ovalle-Marin, A.; Parra-Ruiz, C.; Zamora, O.; Pino, M.; Quitral, V.; Jimenez, P.; Garcia, L.; et al. Extracts of Chilean native fruits inhibit oxidative stress, inflammation and insulin-resistance linked to the pathogenic interaction between adipocytes and macrophages. J. Funct. Foods 2016, 27, 69-83. [CrossRef]

326. Schreckinger, M.E.; Wang, J.; Yousef, G.; Lila, M.A.; De Mejia, E.G. Antioxidant capacity and in vitro inhibition of adipogenesis and inflammation by phenolic extracts of vaccinium floribundum and Aristotelia chilensis. J. Agric. Food Chem. 2010, 58, 8966-8976. [CrossRef]

327. Hidalgo, J. Delphinidin reduces glucose uptake in mice jejunal tissue and human intestinal cells lines through FFA1/GPR40. Int. J. Mol. Sci. 2017, 18, 750. [CrossRef]

328. Christensen, M.; Calanna, S.; Sparre-Ulrich, A.H.; Kristensen, P.L.; Rosenkilde, M.M.; Faber, J.; Purrello, F.; Van Hall, G.; Holst, J.J.; Vilsbøll, T.; et al. Glucose-dependent insulinotropic polypeptide augments glucagon responses to hypoglycemia in Type 1 Diabetes. Diabetes 2014, 64, 72-78. [CrossRef]

329. Miranda-Rottmann, S.; Aspillaga, A.A.; Pérez, D.D.; Vásquez, L.; Martinez, A.L.F.; Leighton, F. Juice and Phenolic Fractions of the BerryAristotelia chilensisInhibit LDL Oxidationin vitroand protect human endothelial cells against oxidative stress. J. Agric. Food Chem. 2002, 50, 7542-7547. [CrossRef]

(C) 2020 by the authors. Licensee MDPI, Basel, Switzerland. This article is an open access article distributed under the terms and conditions of the Creative Commons Attribution (CC BY) license (http://creativecommons.org/licenses/by/4.0/). 